\title{
On the Geometry of Interest Rate Models *
}

\author{
Tomas Björk ${ }^{\dagger}$ \\ Department of Finance \\ Stockholm School of Economics, Box 6501 \\ S-113 83 Stockholm SWEDEN \\ e-mail: tomas.bjork@hhs.se \\ September 19, 2003 \\ Forthcoming in \\ Springer Lecture Notes in Mathematics
}

\begin{abstract}
In this paper, which is a substantial extension of an earlier essay [3], we give an overview of some recent work on the geometric properties of the evolution of the forward rate curve in an arbitrage free bond market. The main problems to be discussed are as follows.

- When is a given forward rate model consistent with a given family of forward rate curves?

- When can the inherently infinite dimensional forward rate process be realized by means of a Markovian finite dimensional state space model.

We consider interest rate models of Heath-Jarrow-Morton type, where the forward rates are driven by a multidimensional Wiener process, and where he volatility is allowed to be an arbitrary smooth functional of the present forward rate curve. Within this framework we give necessary and sufficient conditions for consistency, as well as for the existence of a finite dimensional realization, in terms of the forward rate volatilities. We also study stochastic volatility HJM models, and we provide a systematic method for the construction of concrete realizations.
\end{abstract}

JEL: E43, G13.

Key Words: HJM models, Forward rate curves, interest rate models, factor models, state space models, Markovian realizations.

*SSE/EFI Working Paper Series in Economics and Finance No. 545

${ }^{\dagger} \mathrm{I}$ am highly indebted to B.J. Christensen, C.Landen and L. Svensson for their generosity in letting me use our joint results for this overview. 


\section{Contents}

1 Introduction 4

$1.1 \quad \operatorname{setup} \ldots \ldots \ldots \ldots \ldots \ldots \ldots \ldots$

1.2 The Geometric Point of View . . . . . . . . . . . . . 5

1.3 Main Problems . . . . . . . . . . . . . . . . . . 5

2 A Primer on Linear Realization Theory 7

2.1 Deterministic Forward Rate Volatilities _ . . . . . . . . . . 7

2.2 Finite Dimensional Realizations . . . . . . . . . . . . . . . . . 9

2.3 Economic Interpretation of the State Space . . . . . . . . . . 13

2.4 Connections to Systems and Control Theory . . . . . . . . . . . 14

2.5 Examples . . . . . . . . . . . . . . . . . . . 14

2.6 Notes . . . . . . . . . . . . . . . . . . . . . 15

3 The Consistency Problem 15

3.1 Parameter Recalibration . . . . . . . . . . . . . . . 16

3.2 Invariant Manifolds . . . . . . . . . . . . . . . . . . . . 18

3.3 The Space . . . . . . . . . . . . . . . . . . . . . . . . . 19

3.3.1 The Forward Curve Manifold . . . . . . . . . . . . . . . 19

3.3.2 The Interest Rate Model . . . . . . . . . . . . . . . 20

3.3.3 The Problem . . . . . . . . . . . . . . . . 20

3.4 The Invariance Conditions . . . . . . . . . . . . . . . . 20

3.5 Examples . . . . . . . . . . . . . . . . . . . 24

3.5.1 The Nelson-Siegel Family . . . . . . . . . . . . . . . . . 24

3.5.2 The Hull-White and Ho-Lee Models . . . . . . . . . . . . 24

3.6 The Filipovic State Space Approach to Consistency . . . . . . . . 25

3.7 Notes . . . . . . . . . . . . . . . . . . . . . . . . . . 30

4 The General FDR Problem $\quad 30$

4.1 Setup . . . . . . . . . . . . . . . . . . . 31

4.2 The Geometric problem . . . . . . . . . . . . . . . . 31

4.3 The Main Result . . . . . . . . . . . . . . . . . . . . . . . . . 32 
4.4 Constructing the invariant manifold . . . . . . . . . 35

4.5 Applications . . . . . . . . . . . . . . . . 37

4.5.1 Constant Volatility: Existence of FDRs _ . . . . . . 37

4.5.2 Constant Volatility: Invariant Manifolds . . . . . . . . . 38

4.5.3 Constant Direction Volatility . . . . . . . . . . . . . 41

4.5.4 When is the Short Rate a Markov Process? . . . . . . . . 42

4.6 Notes . . . . . . . . . . . . . . . . . . . . . . . . . . . 44

5 Constructing Realizations $\quad 45$

5.1 The Construction Algorithm . . . . . . . . . . . . . 45

5.2 Deterministic volatility . . . . . . . . . . . . . . . 46

$5.2 .1 \quad$ Ho-Lee . . . . . . . . . . . . . . . . . . . . . . . 49

$5.2 .2 \quad$ Hull-White . . . . . . . . . . . . . . . . . . . . . . . 49

5.3 Deterministic direction volatility $\ldots \ldots \ldots \ldots \ldots$

5.3 .1 The generic case . . . . . . . . . . . . 50

6 The Filipovic and Teichmann Extension 54

7 Stochastic Volatility Models $\quad 55$

7.1 Problem formulation . . . . . . . . . . . . . . . . 57

7.2 Test examples: I. . . . . . . . . . . . . . . . . . . 58

7.3 Finite realizations for general stochastic volatility models . . . . 59

7.3.1 Lie algebra conditions for the existence of an FDR . . . 59

7.3.2 Geometric intuition . . . . . . . . . . . . . . 61

7.4 General orthogonal noise models . . . . . . . . . . . . . 62

7.4.1 Model specification and preliminary results . . . . . . . 62

7.4.2 Necessary conditions . . . . . . . . . . . . . . . . 65

7.4.3 Test examples: II. . . . . . . . . . . . . . . . . . 72

7.4.4 Necessary and sufficient conditions . . . . . . . . . . 73

7.4.5 A simple sufficient condition . . . . . . . . . . . 75

7.4 .6 An example . . . . . . . . . . . . . . . . 78

7.5 Forward rate stochastic volatility models . . . . . . . . . . . 79 
7.5.1 Necessary conditions for orthogonal noise models . . . . 79

7.5.2 Sufficient conditions for the general noise models . . . . . 80

7.5 .3 The scalar case . . . . . . . . . . . . . . . 82

7.5.4 Test examples: III. . . . . . . . . . . . . . . . . . . 84

7.5.5 Construction of realizations . . . . . . . . . . 84

\section{Introduction}

\section{$1.1 \quad$ Setup}

We consider a bond market model (see [4], [33]) living on a filtered probability space $(\Omega, \mathcal{F}, \mathbf{F}, Q)$ where $\mathbf{F}=\left\{\mathcal{F}_{t}\right\}_{t \geq 0}$. The basis is assumed to carry a standard $m$-dimensional Wiener process $W$, and we also assume that the filtration $\mathbf{F}$ is the internal one generated by $W$.

By $p_{t}(x)$ we denote the price, at $t$, of a zero coupon bond maturing at $t+x$, and the forward rates $r_{t}(x)$ are defined by

$$
r_{t}(x)=-\frac{\partial \log p_{t}(x)}{\partial x}
$$

Note that we use the Musiela parameterization, where $x$ denotes the time to maturity. The short rate $R$ is defined as $R_{t}=r_{t}(0)$, and the money account $B$ is given by $B_{t}=\exp \left\{\int_{0}^{t} R_{s} d s\right\}$. The model is assumed to be free of arbitrage in the sense that the measure $Q$ above is a martingale measure for the model. In other words, for every fixed time of maturity $T \geq 0$, the process $Z_{t}(T)=$ $p_{t}(T-t) / B_{t}$ is a $Q$-martingale.

Let us now consider a given forward rate model of the form

$$
\left\{\begin{aligned}
d r_{t}(x) & =\beta_{t}(x) d t+\sigma_{t}(x) d W, \\
r_{0}(x) & =r_{0}^{o}(x) .
\end{aligned}\right.
$$

where, for each $x, \beta(x)$ and $\sigma(x)$ are given optional processes. The initial curve $\left\{r_{0}^{o}(x) ; x \geq 0\right\}$ is taken as given. It is interpreted as the observed forward rate curve.

The standard Heath-Jarrow-Morton drift condition ([26]) can easily be transferred to the Musiela parameterization. The result (see [10], [32]) is as follows.

Proposition 1.1 (The Forward Rate Equation) Under the martingale measure $Q$ the $r$-dynamics are given by

$$
\begin{aligned}
d r_{t}(x) & =\left\{\frac{\partial}{\partial x} r_{t}(x)+\sigma_{t}(x) \int_{0}^{x} \sigma_{t}(u)^{\star} d u\right\} d t+\sigma_{t}(x) d W_{t}, \\
r_{0}(x) & =r_{0}^{o}(x) .
\end{aligned}
$$


where $^{\star}$ denotes transpose.

\subsection{The Geometric Point of View}

At a first glance it is natural to view the forward rate equation (2) as an infinite system of SDEs: We have one equation for each fixed $x$, so we are handling a continuum of SDEs.

An alternative, more geometrically oriented, view of (2) is to regard it as a single equation for an infinite dimensional object. The infinite dimensional object under study is the forward rate curve, i.e. the curve $x \longmapsto r_{t}(x)$. Denoting the forward rate curve at time $t$ by $r_{t}$, and the entire forward rate curve process by $r$, we thus take the point of view that $r$ is a process evolving in some infinite dimensional function space $\mathcal{H}$ of forward rate curves. For a fixed $t$ we will thus view each outcome of $r_{t}$ as a vector (or point) in $\mathcal{H}$. In the same way, the volatility process $\sigma=\left[\sigma_{1}, \ldots, \sigma_{m}\right]$ is viewed as a process evolving in $\mathcal{H}^{m}$, so that for each $t$ the outcome of $\sigma_{t}=\left[\sigma_{1 t}, \ldots, \sigma_{m t}\right]$ is regarded as a point in $\mathcal{H}^{m}$.

In order to avoid detailed technical discussions at this preliminary level we postpone the precise definition of $\mathcal{H}$, as well as the necessary technical conditions on $\sigma$, until Section 3.3. For the time being the reader is asked to think (loosely) of $\mathcal{H}$ as a space of $C^{\infty}$ functions and to assume that all SDEs appearing in the text do admit unique strong solutions.

In order to emphasize the geometric point of view, can now rewrite the forward rate equation (2) as

$$
\begin{aligned}
d r_{t} & =\left\{\mathbf{F} r_{t}+\sigma_{t} \mathbf{H} \sigma_{t}\right\} d t+\sigma_{t} d W_{t} \\
r_{0} & =r^{0}
\end{aligned}
$$

where the operators $\mathbf{F}$ and $\mathbf{H}$, both defined $\mathcal{H}$, are given by

$$
\begin{aligned}
\mathbf{F} & =\frac{\partial}{\partial x}, \\
{[\mathbf{H} f](x) } & =\int_{0}^{x} f(s) d s, \quad \text { for } f \in \mathcal{H} .
\end{aligned}
$$

and where we use the obvious interpretation $\mathbf{H} \sigma_{t}=\left[\mathbf{H} \sigma_{1 t}, \ldots, \mathbf{H} \sigma_{m t}\right]$.

\subsection{Main Problems}

Suppose now that we are give a concrete model $\mathcal{M}$ within the above framework, i.e. suppose that we are given a concrete specification of the volatility process $\sigma$. We now formulate a couple of natural problems:

1. Take, in addition to $\mathcal{M}$, also as given a parameterized family $\mathcal{G}$ of forward rate curves. Under which conditions is the family $\mathcal{G}$ consistent with the 
dynamics of $\mathcal{M}$ ? Here consistency is interpreted in the sense that, given an initial forward rate curve in $\mathcal{G}$, the interest rate model $\mathcal{M}$ will only produce forward rate curves belonging to the given family $\mathcal{G}$.

2. When does the given, inherently infinite dimensional, interest rate model $\mathcal{M}$ admit a finite dimensional realization? More precisely, we seek conditions under which the forward rate process $r_{t}(x)$ induced by the model $\mathcal{M}$, can be realized by a system of the form

$$
\begin{aligned}
d Z_{t} & =a\left(Z_{t}\right) d t+b\left(Z_{t}\right) d W_{t}, \\
r_{t}(x) & =G\left(Z_{t}, x\right) .
\end{aligned}
$$

where $Z$ (interpreted as the state vector process) is a finite dimensional diffusion, $a(z), b(z)$ and $G(z, x)$ are deterministic functions and $W$ is the same Wiener process as in (2). Expressed in other terms, we thus wish to investigate under what conditions the HJM model is generated by a finite dimensional Markovian state space model.

As will be seen below, these two problems are intimately connected, and the main purpose of this chapter, which is a substantial extension of a previous paper [3], is to give an overview of some recent work in this area. The text is based on [6], [5], [9], [7], and [8], but the presentation given below is more focused on geometric intuition than the original articles, where full proofs, technical details and further results can be found. In the analysis below we use ideas from systems and control theory (see [30]) as well as from nonlinear filtering theory (see [12]). References to the literature will sometimes be given in the text, but will also be summarized in the Notes at the end of each section.

It should be noted that the functional analytical framework of the entire theory has recently been improved in a quite remarkable way by Filipovic and Teichmann. In a series of papers these authors have considerably extended the Hilbert space framework of the papers mentioned above. In doing so, they have also clarified many structural problems and derived a large number of concrete results. However: a full understanding of these extensions require a high degree of detailed technical knowledge in analysis on Frechet spaces so the scope of the present paper prohibits us from doing complete justice to this beautiful part of the theory. The interested reader is referred to the original papers [23], [24], and [25].

The organization of the text is as follows. In Section 2 we treat the relatively simple case of linear realizations. Section 3 is devoted to a study of the general consistency problem, including a primer on the Filpovic inverse consistency theory, and in Section 4 we use the consistency results from Section 3 in order to give a fairly complete picture of the nonlinear realization problem. The problem of actually constructing a concrete realization is treated in Section 5, in Section 6 we discuss very briefly the Filipovic-Teicmann extensions, and in Section 7 we extend the theory to include stochastic volatility models. 


\section{A Primer on Linear Realization Theory}

In the general case, the forward rate equation (4) is a highly nonlinear infinite dimensional SDE but, as can be expected, the special case of linear dynamics is much easier to handle. In this section we therefore concentrate on linear forward rate models, and look for finite dimensional linear realizations. Almost all geometric ideas presented in this chapter will then be generalized to the nonlinear case studied later in the text.

\subsection{Deterministic Forward Rate Volatilities}

For the rest of the section we only consider the case when the volatility process $\sigma_{t}(x)=\left[\sigma_{1 t}(x), \ldots, \sigma_{m t}(x)\right]$ is a deterministic function $\sigma(x)$ of $x$ only.

Assumption 2.1 Each component $\sigma_{i}$, of the volatility process $\sigma$ is a deterministic vector in $\mathcal{H}$ for $i=1, \ldots, m$. Equivalently, The volatility $\sigma$ is a $C^{\infty}$ mapping $\sigma: R_{+} \rightarrow R^{m}$.

Under this assumption, the forward rate equation (4) takes the form

$$
d r_{t}=\left\{\mathbf{F}_{t}+D\right\} d t+\sigma d W_{t},
$$

where the function $D$, which we view as a vector $D \in \mathcal{H}$, is defined by

$$
D(x)=\sigma(x) \int_{0}^{x} \sigma(s)^{\star} d s .
$$

The point to note here is that, because of our choice of a deterministic volatility $\sigma(x)$, the forward rate equation (10) is a linear (or rather affine) SDE evolving in the infinite dimensional function space $\mathcal{H}$.

Because of the linear structure of the equation (albeit in infinite dimensions) we expect to be able to provide an explicit solution of (10). We now recall that a scalar equation of the form

$$
d y_{t}=\left[a y_{t}+b\right] d t+c d W_{t}
$$

has the solution

$$
y_{t}=e^{a t} y_{0}+\int_{0}^{t} e^{a(t-s)} b d s+\int_{0}^{t} e^{a(t-s)} c d W_{s},
$$

and we are thus led to conjecture that the solution to (10) is given by the formal expression

$$
r_{t}=e^{\mathbf{F} t} r_{0}+\int_{0}^{t} e^{\mathbf{F}(t-s)} D d s+\int_{0}^{t} e^{\mathbf{F}(t-s)} \sigma d W_{s}
$$


We now have to make precise mathematical sense of the formal exponent $e^{\mathbf{F} t}$. From the context it is clear that it acts on vectors in $\mathcal{H}$, i.e. on real valued $C^{\infty}$ functions. It is in fact an operator $e^{\mathbf{F} t}: \mathcal{H} \rightarrow \mathcal{H}$ and we have to figure out how it acts. From the usual series expansion of the exponential function one is led to write

$$
e^{\mathbf{F} t} f=\sum_{n=0}^{\infty} \frac{t^{n}}{n !} F^{n} f
$$

In our case $F^{n}=\frac{\partial^{n}}{\partial x^{n}}$, so we have

$$
\left[e^{\mathbf{F} t} f\right](x)=\sum_{n=0}^{\infty} \frac{t^{n}}{n !} \frac{\partial^{n} f}{\partial x^{n}}(x)
$$

This is, however, just a Taylor series expansion of $f$ around the point $x$, so for analytic $f$ we have $\left[e^{\mathbf{F} t} f\right](x)=f(x+t)$. We have in fact the following precise result (which can be proved rigorously).

Proposition 2.1 The operator $\mathbf{F}$ is the infinitesimal generator of the semigroup of left translations, i.e. for any $f \in \mathcal{H}$ (and in fact for any continuous $f$ ) we have

$$
\left[e^{\mathbf{F} t} f\right](x)=f(t+x) .
$$

Furthermore, the solution of the forward rate equation (10) is given by

$$
r_{t}=e^{\mathbf{F} t} r^{o}+\int_{0}^{t} e^{\mathbf{F}(t-s)} D d s+\int_{0}^{t} e^{\mathbf{F}(t-s)} \sigma d W_{s}
$$

or, written in component form, by

$$
r_{t}(x)=r^{o}(x+t)+\int_{0}^{t} D(x+t-s) d s+\int_{0}^{t} \sigma(x+t-s) d W_{s} .
$$

From (15) it is clear by inspection that we may write the solution of the forward rate equation (10) as

$$
\begin{aligned}
r_{t} & =q_{t}+\delta_{t}, \\
d q_{t} & =\mathbf{F} q_{t} d t+\sigma d W_{t}, \\
q_{0} & =0
\end{aligned}
$$

where $\delta$ is given by

$$
\delta_{t}=e^{\mathbf{F} t} r^{o}+\int_{0}^{t} e^{\mathbf{F}(t-s)} D d s,
$$

or on component form

$$
\delta_{t}(x)=r^{o}(x+t)+\int_{0}^{t} D(x+t-s) d s
$$


Since $\delta_{t}(x)$ is not affected by the input $W$, we see that the problem of finding a realization for the term structure system (10) is equivalent to that of finding a realization for (18)-(19). Since we have a linear dynamical system it seems natural to look for linear realizations and we are thus led to the following definition.

Definition 2.1 A triple $[A, B, C(x)]$, where $A$ is an $(n \times n)$-matrix, $B$ is an $(n \times m)$-matrix and $C$ is an $n$ dimensional row vector function, is called an $n$-dimensional realization of the systems (18) if $q$ has the representation

$$
\begin{aligned}
q_{t}(x) & =C(x) Z_{t}, \\
d Z_{t} & =A Z_{t} d t+B d W_{t}, \\
Z_{0} & =0 .
\end{aligned}
$$

Our main problems are now as follows.

- Consider an a priori given volatility structure $\sigma(x)$.

- When does there exists a finite dimensional realization?

- If there exists a finite dimensional realization, what is the minimal dimension?

- How do we construct a minimal realization from knowledge of $\sigma$ ?

\subsection{Finite Dimensional Realizations}

In this section we will investigate the existence of a finite dimensional realization (FDR) from a geometric point of view. There are also a number of other ways to attack the problem, but it is in fact the geometrical point of view which later in the text will be generalized to the nonlinear case. The discussion will be rather informal and some technical questions are sidestepped.

We recall the $q$-equation (18) as

$$
\begin{aligned}
d q_{t} & =\mathbf{F} q_{t} d t+\sigma d W_{t} \\
q_{0} & =0
\end{aligned}
$$

Expressing the operator exponential $e^{F(t-s)}$ as a power series, and using Proposition 2.1 , we may write the solution to $(25)$ as

$$
q(t)=\int_{0}^{t} \sum_{0}^{\infty} \frac{(t-s)^{n}}{n !} \mathbf{F}^{n} \sigma d W(s) .
$$


From this expression we see that, for each $t$, the random vector $q_{t}$ is in fact given as a random infinite linear combination of the (deterministic) vectors $\sigma, \mathbf{F} \sigma, \mathbf{F}^{2} \sigma, \ldots$. Thus we see that the $q$-process will in fact evolve in the (deterministic) subspace $\mathcal{R} \subseteq \mathcal{H}$ defined by

$$
\mathcal{R}=\operatorname{span}\left[\sigma, \mathbf{F} \sigma, \mathbf{F}^{2} \sigma, \ldots\right] .
$$

The subspace $\mathcal{R}$ is thus invariant under the action of the $q$ process, and it is rather obvious that it is in fact the minimal (under inclusion) invariant subspace for $q$.

The obvious conjecture is that there exists an FDR if and only if $\mathcal{R}$ is finite dimensional. This conjecture is in fact correct and we have the following main result.

Proposition 2.2 Consider a given volatility function

$$
\sigma=\left[\sigma_{1}, \cdots, \sigma_{m}\right] .
$$

Then there exists an FDR if and only if,

$$
\operatorname{dim}(\mathcal{R})<\infty,
$$

with $\mathcal{R}$ defined by

$$
\mathcal{R}=\operatorname{span}\left[\mathbf{F}^{k} \sigma_{i} ; i=1, \cdots, m ; k=0,1, \cdots\right] .
$$

Furthermore; the minimal dimension of an FDR, also known as the McMillan degree, is given by $\operatorname{dim}(\mathcal{R})$.

Proof. For brevity we only give the proof for the case $m=1$. The proof for general case is almost identical. Assume first that there exists an $n$-dimensional FDR of the form

$$
q_{t}(x)=C(x) Z_{t},
$$

with $Z$-dynamics as in (24), and with

$$
C(x)=\left[C_{1}(x), \ldots, C_{n}(x)\right] .
$$

Writing this as

$$
q_{t}=C Z_{t},
$$

it is now obvious that the finite dimensional subspace of $\mathcal{H}$ spanned by the vectors $C_{1}, \ldots, C_{n}$ will in fact be invariant and thus contain $\mathcal{R}$ (since $\mathcal{R}$ is minimal invariant). Thus the existence of an FDR implies the finite dimensionality of $\mathcal{R}$.

Conversely, assume that $\mathcal{R}$ is $n$-dimensional. We now prove the existence of an FDR by actually constructing an explicit realization of the form (22)-(24). The 
finite dimensionality of $\mathcal{R}$ implies that, for some integer $n$, there exists a linear relation of the form

$$
\mathbf{F}^{n} \sigma=\sum_{i=0}^{n-1} \gamma_{i} \mathbf{F}^{i} \sigma
$$

where $\gamma_{0}, \ldots, \gamma_{n-1}$ are real numbers. Without loss of generality we may assume that $n$ is the smallest integer with this property, so that the vectors $\sigma, \mathbf{F} \sigma, \ldots \mathbf{F}^{n-1} \sigma$ are linearly independent and span $\mathcal{R}$.

Since $\sigma, \mathbf{F} \sigma, \ldots, \mathbf{F}^{n-1} \sigma$ is a basis for the invariant subspace $\mathcal{R}$ we can write

$$
q_{t}=\sum_{i=0}^{n-1} Z_{t}^{i} \mathbf{F}^{i} \sigma .
$$

where the processes $Z^{0}, \ldots, Z^{n-1}$ are the coordinate processes of $q$ for the given basis. We now want to find the dynamics of $Z=\left[Z^{0}, \ldots, Z^{n-1}\right]$ so we make the Ansatz

$$
d Z_{t}^{i}=\alpha_{i}\left(Z_{t}\right) d t+\beta_{i}\left(Z_{t}\right) d W_{t}, \quad i=0, \ldots, n-1,
$$

and the problem is to identify the unknown functions $\alpha$ and $\beta$.

Our strategy for finding $\alpha$ and $\beta$ is as follows.

- Compute $d q$ from (33)-(34).

- Compare the expression thus obtained with the original $q$ dynamics given by

$$
d q_{t}=\mathbf{F} q_{t} d t+\sigma d W_{t}
$$

- Identify the coefficients.

From (33)-(34) and using the notation

$$
\sigma^{(i)}=\mathbf{F}^{i} \sigma,
$$

we obtain

$$
d q_{t}=\left(\sum_{i=0}^{n-1} \alpha_{i}\left(Z_{t}\right) \sigma^{(i)}\right) d t+\left(\sum_{i=0}^{n-1} \beta_{i}\left(Z_{t}\right) \sigma^{(i)}\right) d W_{t} .
$$

We now want to compare this expression with the dynamics in (35). To do this we first use (33) to obtain

$$
\mathbf{F} q_{t}=\sum_{i=0}^{n-1} Z_{t}^{i} \mathbf{F}^{i+1} \sigma=\sum_{i=0}^{n-1} Z_{t}^{i} \sigma^{i+1}=\sum_{i=1}^{n} Z_{t}^{i-1} \sigma^{i} .
$$


After inserting (32) into (37) and collecting terms we have

$$
\mathbf{F} q_{t}=\sum_{i=0}^{n-1} c_{i}\left(Z_{t}\right) \sigma^{i},
$$

where

$$
c_{i}(Z)=Z^{i-1}+\gamma_{i} Z^{n-1}, \quad i=0, \ldots, n-1,
$$

with the convention $Z^{-1}=0$. We may thus write the $q$ dynamics in (35) as

$$
d q_{t}=\left(\sum_{i=0}^{n-1} c_{i}\left(Z_{t}\right) \sigma^{i}\right) d t+\sigma^{0} d W_{t} .
$$

We may now identify coefficients by comparing (38) with (40) to obtain

$$
\begin{aligned}
\beta_{0}(Z) & =1, \\
\beta_{i} & =0, \quad i=1, \ldots, n-1 .
\end{aligned}
$$

and

$$
\alpha_{i}(Z)=c_{i}(Z), \quad i=0, \ldots, n-1 .
$$

with $c_{i}$ as in (39). We have thus derived the explicit realization

$$
q_{t}=C Z_{t},
$$

where

$$
C=\left[\sigma, \mathbf{F} \sigma, \ldots, \mathbf{F}^{n-1} \sigma\right],
$$

and where the $Z$ dynamics are given by

$$
\begin{aligned}
d Z_{t}^{0} & =\gamma_{0} Z_{t}^{n-1} d t+d W_{t}, \\
d Z_{t}^{i} & =\left(Z_{t}^{i-1}-\gamma_{i} Z_{t}^{n-1}\right) d t, \quad i=1, \ldots, n-1 .
\end{aligned}
$$

We note in passing that the proof above, apart from proving the existence result, also provides us with the concrete realization (43)-(45). In the proof this is only done for the case of a scalar Wiener process, but the method can easily be extended to the multi-dimensional case. See [7] for worked out examples.

We now go on to find a more explicit characterization of condition (28). Recalling that the operator $\mathbf{F}$ is defined as $\mathbf{F}=\frac{\partial}{\partial x}$, we see from Proposition 2.2 that the forward rate system admits an FDR if and only if the space spanned by the components of $\sigma$ and all their derivatives is finite dimensional. In other words; there exists an FDR if and only if the components of $\sigma$ satisfy a linear system of ODEs with constant coefficients. This leads us to the topic of quasi-exponential functions. 
Definition 2.2 A quasi-exponential (or $Q E$ ) function is by definition any function of the form

$$
f(x)=\sum_{i} e^{\lambda_{i} x}+\sum_{j} e^{\alpha_{i} x}\left[p_{j}(x) \cos \left(\omega_{j} x\right)+q_{j}(x) \sin \left(\omega_{j} x\right)\right],
$$

where $\lambda_{i}, \alpha_{1}, \omega_{j}$ are real numbers, whereas $p_{j}$ and $q_{j}$ are real polynomials.

QE functions will turn up over and over again, so we list some simple well known properties.

Lemma 2.1 The following hold for the quasi-exponential functions.

- A function is $Q E$ if and only if it is a component of the solution of a vector valued linear ODE with constant coefficients.

- A function is $Q E$ if and only if it can be written as $f(x)=c e^{A x} b$. where $c$ is a row vector, $A$ is a square matrix and $b$ is a column vector.

- If $f$ is $Q E$, then $f^{\prime}$ is $Q E$.

- If $f$ is $Q E$, then its primitive function is $Q E$.

- If $f$ and $g$ are $Q E$, then $f g$ is $Q E$.

We can thus restate Proposition 2.2.

Proposition 2.3 The forward rate equation admits a finite dimensional realization if and only of each component of $\sigma$ is quasi-exponential.

\subsection{Economic Interpretation of the State Space}

In general, the state space of a realization of a given system has no concrete (e.g. economic) interpretation. In the case of the forward rate equation, however, the states of the minimal realization turn out to have a simple economic interpretation.

Proposition 2.4 Assume that $\mathcal{R}$ is $n$-dimensional, so that the existence of an FDR is guaranteed. Then, for any minimal realization, i.e. a realization with an $n$-dimensional state vector $Z$, there will exist an affine transformation mapping the state vector into a vector of benchmark forward rates.

The moral of this is that, in a minimal realization, you can always choose your state variables as a fixed set of forward rates. It can also be shown that the maturities of the benchmark forward rates can be chosen without restrictions.

For precise statements, proofs and examples, see [6]. 


\subsection{Connections to Systems and Control Theory}

The geometric ideas of the previous section are in fact standard in the theory of mathematical systems and control. To see this, consider again the equation

$$
\begin{aligned}
d q_{t} & =\mathbf{F} q_{t} d t+\sigma d W_{t} \\
q_{0} & =0
\end{aligned}
$$

Let us now formally "divide by $d t$ ", which gives us

$$
\frac{d q_{t}}{d t}=\mathbf{F} q_{t}+\sigma \frac{d W_{t}}{d t}
$$

where the formal time derivative $\frac{d W_{t}}{d t}$ is interpreted as "white noise". We interpret this equation as an input-output system where the random input signal $t \longmapsto \frac{d W_{t}}{d t}$ is transformed into the infinite dimensional output signal $t \longmapsto q_{t}$. We thus view the equation as a stochastic version of the following controlled ODE

$$
\begin{aligned}
\frac{d q_{t}}{d t} & =\mathbf{F} q_{t}+\sigma u_{t} \\
q_{0} & =0
\end{aligned}
$$

where $u$ is a deterministic input signal (which in our case is replaced by white noise). Generally speaking, tricks like this does not work directly, since we are ignoring the difference between standard differential calculus, which is used to analyze (49), and Itô calculus which we use when dealing with SDEs. In this case, however, because of the linear structure, the second order Itô term will not come into play, so we are safe. (See the discussion in Section 3.4 around the Stratonovich integral for how to treat the nonlinear situation.)

The reader who is familiar with systems and control theory (see [11]) will now recognize the space $\mathcal{R}$ above as the reachable subspace of the control system (49). Not surprisingly, there is also a frequency domain approach to our realization problem. See [6] for details.

\subsection{Examples}

We now give some simple illustrations of the theory. We only consider the case of a scalar driving Wiener process.

Example 2.1 $\sigma(x)=\sigma e^{-a x}$

We consider a model driven by a one-dimensional Wiener process, having the forward rate volatility structure

$$
\sigma(x)=\sigma e^{-a x},
$$


where $\sigma$ in the right hand side denotes a constant. (The reader will probably recognize this example as the Hull-White model.) We start by determining $\mathcal{R}$ which in this case is defined as

$$
\mathcal{R}=\operatorname{span}\left[\frac{d^{k}}{d x^{k}} \sigma e^{-a x} ; k \geq 0\right] .
$$

It is obvious that $\mathcal{R}$ is one-dimensional, so we can expect to find a one-dimensional realization. The existence of an FDR could of course also have been seen directly by observing that $\sigma$ is quasi-exponential. Since we have $\mathbf{F} \sigma=-a \sigma$ we see that, in the notation of (32) we have $\gamma_{0}=-a$. Thus denoting the single state variable $Z^{0}$ by $Z$, we may use (43)-(45) to obtain the realization

$$
\begin{aligned}
q_{t}(x) & =e^{-a x} Z_{t}, \\
d Z_{t} & =-a Z_{t} d t+d W_{t} .
\end{aligned}
$$

A full realization of the forward rate process $r_{t}$ is then obtained from (17) as

$$
r_{t}(x)=\sigma e^{-a x} Z_{t}+r^{0}(x+t)+\frac{1}{2} \sigma^{2} e^{-2 a x}\left\{e^{-2 a t}-1\right\} .
$$

Example 2.2 $\sigma(x)=e^{-x^{2}}$

In this example $\mathcal{R}$ is given by

$$
\mathcal{R}=\operatorname{span}\left[\frac{d^{k}}{d x^{k}} e^{-x^{2}}, k \geq 0\right]=\operatorname{span}\left[x^{k} e^{-x^{2}}, k \geq 0\right],
$$

which is easily seen to be infinite dimensional. Thus we see that in this case there exists no finite dimensional linear realization. We will return to this example later and we will in fact prove that neither does there exists a non-linear FDR.

\subsection{Notes}

This section is to a large extent based on [6] where, however, the focus is more on the frequency domain approach. The first paper to appear in this area was to our knowledge the seminal preprint [32], where the Musiela parameterization is introduced and the space $\mathcal{R}$ is discussed in some detail. Because of the linear structure, the theory above is closely connected to (and in a sense inverse to) the theory of affine term structures developed in [17]. The standard reference on infinite dimensional SDEs is [16], where one also can find a presentation of the connections between control theory and infinite dimensional linear stochastic equations.

\section{The Consistency Problem}

We now turn to a more serious study of the geometric properties of the forward rate equation in the general nonlinear case. We begin by studying when a given 
submanifold of forward rate curves is consistent (a precise definition will be given below) with a given interest rate model. This problem is of interest from an applied as well as from a theoretical point of view. In particular we will use the results from this section to analyze problems about existence of finite dimensional factor realizations for interest rate models on forward rate form. Invariant manifolds are, however, also of interest in their own right, so we begin by discussing a concrete problem which naturally leads to the invariance concept.

\subsection{Parameter Recalibration}

A standard procedure when dealing with concrete interest rate models on a high frequency (say, daily) basis can be described as follows:

1. At time $t=0$, use market data to fit (calibrate) the model to the observed bond prices.

2. Use the calibrated model to compute prices of various interest rate derivatives.

3. The following day $(t=1)$, repeat the procedure in 1 . above in order to recalibrate the model, etc..

To carry out the calibration in step 1 . above, the analyst typically has to produce a forward rate curve $\left\{r^{\circ}(x) ; x \geq 0\right\}$ from the observed data. However, since only a finite number of bonds actually trade in the market, the data consist of a discrete set of points, and a need to fit a curve to these points arises. This curve-fitting may be done in a variety of ways. One way is to use splines, but also a number of parameterized families of smooth forward rate curves have become popular in applications - the most well-known probably being the Nelson-Siegel (see [34]) family. Once the curve $\left\{r^{o}(x) ; x \geq 0\right\}$ has been obtained, the parameters of the interest rate model may be calibrated to this.

Now, from a purely logical point of view, the recalibration procedure in step 3. above is of course slightly nonsensical: If the interest rate model at hand is an exact picture of reality, then there should be no need to recalibrate. The reason that everyone insists on recalibrating is of course that any model in fact only is an approximate picture of the financial market under consideration, and recalibration allows incorporating newly arrived information in the approximation. Even so, the calibration procedure itself ought to take into account that it will be repeated. It appears that the optimal way to do so would involve a combination of time series and cross-section data, as opposed to the purely cross-sectional curve-fitting, where the information contained in previous curves is discarded in each recalibration.

The cross-sectional fitting of a forward curve and the repeated recalibration is thus, in a sense, a pragmatic and somewhat non-theoretical endeavor. Nonetheless, there are some nontrivial theoretical problems to be dealt with in this 
context, and the problem to be studied in this section concerns the consistency between, on the one hand, the dynamics of a given interest rate model, and, on the other hand, the forward curve family employed.

What, then, is meant by consistency in this context? Assume that a given interest rate model $\mathcal{M}$ (e.g. the Hull-White model) in fact is an exact picture of the financial market. Now consider a particular family $\mathcal{G}$ of forward rate curves (e.g. the Nelson-Siegel family) and assume that the interest rate model is calibrated using this family. We then say that the pair $(\mathcal{M}, \mathcal{G})$ is consistent (or, that $\mathcal{M}$ and $\mathcal{G}$ are consistent) if all forward curves which may be produced by the interest rate model $\mathcal{M}$ are contained within the family $\mathcal{G}$. Otherwise, the pair $(\mathcal{M}, \mathcal{G})$ is inconsistent.

Thus, if $\mathcal{M}$ and $\mathcal{G}$ are consistent, then the interest rate model actually produces forward curves which belong to the relevant family. In contrast, if $\mathcal{M}$ and $\mathcal{G}$ are inconsistent, then the interest rate model will produce forward curves outside the family used in the calibration step, and this will force the analyst to change the model parameters all the time - not because the model is an approximation to reality, but simply because the family does not go well with the model.

Put into more operational terms this can be rephrased as follows.

- Suppose that you are using a fixed interest rate model $\mathcal{M}$. If you want to do recalibration, then your family $\mathcal{G}$ of forward rate curves should be chosen is such a way as to be consistent with the model $\mathcal{M}$.

Note however that the argument also can be run backwards, yielding the following conclusion for empirical work.

- Suppose that a particular forward curve family $\mathcal{G}$ has been observed to provide a good fit, on a day-to-day basis, in a particular bond market. Then this gives you modeling information about the choice of an interest rate model in the sense that you should try to use/construct an interest rate model which is consistent with the family $\mathcal{G}$.

We now have a number of natural problems to study.

I Given an interest rate model $\mathcal{M}$ and a family of forward curves $\mathcal{G}$, what are necessary and sufficient conditions for consistency?

II Take as given a specific family $\mathcal{G}$ of forward curves (e.g. the Nelson-Siegel family). Does there exist any interest rate model $\mathcal{M}$ which is consistent with $\mathcal{G}$ ?

III Take as given a specific interest rate model $\mathcal{M}$ (e.g. the Hull-White model). Does there exist any finitely parameterized family of forward curves $\mathcal{G}$ which is consistent with $\mathcal{M}$ ? 
In this section we will mainly address problem (I) above. Problem II has been studied, for special cases, in [19], [20], whereas Problem III can be shown (see Proposition 4.1) to be equivalent to the problem of finding a finite dimensional factor realization of the model $\mathcal{M}$ and we provide a fairly complete solution in Section 4.

\subsection{Invariant Manifolds}

We now move on to give precise mathematical definition of the consistency property discussed above, and this leads us to the concept of an invariant manifold.

Definition 3.1 (Invariant manifold) Take as given the forward rate process dynamics (2). Consider also a fixed family (manifold) of forward rate curves $\mathcal{G}$. We say that $\mathcal{G}$ is locally invariant under the action of $r$ if, for each point $(s, r) \in R_{+} \times \mathcal{G}$, the condition $r_{s} \in \mathcal{G}$ implies that $r_{t} \in \mathcal{G}$, on a (possibly random) time interval with positive length. If $r$ stays forever on $\mathcal{G}$, we say that $\mathcal{G}$ is globally invariant.

The purpose of this section is to characterize invariance in terms of local characteristics of $\mathcal{G}$ and $\mathcal{M}$, and in this context local invariance is the best one can hope for. In order to save space, local invariance will therefore be referred to as invariance.

To get some intuitive feeling for the invariance concepts one can consider the following two-dimensional deterministic system

$$
\begin{aligned}
\frac{d y_{1}}{d t} & =y_{2}, \\
\frac{d y_{2}}{d t} & =-y_{1} .
\end{aligned}
$$

For this system it is obvious that the unit circle $\mathcal{C}=\left\{\left(y_{1}, y_{2}\right): y_{1}^{2}+y_{2}^{2}=1\right\}$ is globally invariant, i.e. if we start the system on $\mathcal{C}$ it will stay forever on $\mathcal{C}$. The 'upper half' of the circle, $\mathcal{C}_{u}=\left\{\left(y_{1}, y_{2}\right): y_{1}^{2}+y_{2}^{2}=1, y_{2}>0\right\}$, is on the other hand only locally invariant, since the system will leave $\mathcal{C}_{u}$ at the point $(1,0)$. This geometric situation is in fact the generic one also for our infinite dimensional stochastic case. The forward rate trajectory will never leave a locally invariant manifold at a point in the relative interior of the manifold. Exit from the manifold can only take place at the relative boundary points. We have no general method for determining whether a locally invariant manifold also is globally invariant or not. Problems of this kind has to be solved separately for each particular case. 


\subsection{The Space}

In order to study the consistency problem we need (see Remark 3.1 below) a very regular space to work in.

Definition 3.2 Consider a fixed real number $\gamma>0$. The space $\mathcal{H}_{\gamma}$ is defined as the space of all infinitely differentiable functions

$$
r: R_{+} \rightarrow R
$$

satisfying the norm condition $\|r\|_{\gamma}<\infty$. Here the norm is defined as

$$
\|r\|_{\gamma}^{2}=\sum_{n=0}^{\infty} 2^{-n} \int_{0}^{\infty}\left(\frac{d^{n} r}{d x^{n}}(x)\right)^{2} e^{-\gamma x} d x .
$$

Note that $\mathcal{H}$ is not a space of distributions, but a space of functions. We will often suppress the subindex $\gamma$. With the obvious inner product $\mathcal{H}$ is a pre-Hilbert space, and in [9] the following result is proved.

Proposition 3.1 The space $\mathcal{H}$ is a Hilbert space, i.e. it is complete. Furthermore, every function in the space is fact real analytic, and can thus be uniquely extended to a holomorphic function in the entire complex plane.

Remark 3.1 The reason for our choice of $\mathcal{H}$ as the underlying space, is that the linear operator $\mathbf{F}=d / d x$ is bounded in this space. Together with the assumptions above, this implies that both $\mu$ and $\sigma$ are smooth vector fields on $\mathcal{H}$, thus ensuring the existence of a strong local solution to the forward rate equation for every initial point $r^{o} \in \mathcal{H}$.

\subsubsection{The Forward Curve Manifold}

We consider as given a mapping

$$
G: \mathcal{Z} \rightarrow \mathcal{H}
$$

where the parameter space $\mathcal{Z}$ is an open connected subset of $R^{d}$, i.e. for each parameter value $z \in \mathcal{Z} \subseteq R^{d}$ we have a curve $G(z) \in \mathcal{H}$. The value of this curve at the point $x \in R_{+}$will be written as $G(z, x)$, so we see that $G$ can also be viewed as a mapping

$$
G: \mathcal{Z} \times R_{+} \rightarrow R
$$

The mapping $G$ is thus a formalization of the idea of a finitely parameterized family of forward rate curves, and we now define the forward curve manifold as the set of all forward rate curves produced by this family.

Definition 3.3 The forward curve manifold $\mathcal{G} \subseteq \mathcal{H}$ is defined as

$$
\mathcal{G}=\operatorname{Im}(G)
$$




\subsubsection{The Interest Rate Model}

We consider a given volatility $\sigma$ of the form

$$
\sigma: \mathcal{H} \times R_{+} \rightarrow R^{m} .
$$

In other words, $\sigma(r, x)$ is a functional of the infinite dimensional $r$-variable, and a function of the real variable $x$. An alternative, and more profitable, way of viewing a component $\sigma_{i}$ is to see it as a mapping where point $r \in \mathcal{H}$ is mapped to the real valued function $\sigma_{i}(r, \cdot)$. We will in fact assume that this real valued function is a member of $\mathcal{H}$, which means that we can view each component $\sigma_{i}$ as a vector field $\sigma_{i}: \mathcal{H} \rightarrow \mathcal{H}$ on the space $\mathcal{H}$. Denoting the forward rate curve at time $t$ by $r_{t}$ we then have the following forward rate equation.

$$
d r_{t}(x)=\left\{\frac{\partial}{\partial x} r_{t}(x)+\sigma\left(r_{t}, x\right) \int_{0}^{x} \sigma\left(r_{t}, u\right)^{\star} d u\right\} d t+\sigma\left(r_{t}, x\right) d W_{t} .
$$

Remark 3.2 For notational simplicity we have assumed that the $r$-dynamics are time homogenous. The case when $\sigma$ is of the form $\sigma(t, r, x)$ can be treated in exactly the same way. See [5].

We obviously need some regularity assumptions and these will be collected in Assumption 3.1 below. See [5] for further technical details.

\subsubsection{The Problem}

Our main problem is the following.

- Suppose that we are given

- A volatility $\sigma$, specifying an interest rate model $\mathcal{M}$ as in (55)

- A mapping $G$, specifying a forward curve manifold $\mathcal{G}$.

- Is $\mathcal{G}$ then invariant under the action of $r$ ?

\subsection{The Invariance Conditions}

In order to study the invariance problem from a geometrical point of view we introduce some compact notation.

Definition 3.4 We define $\mathbf{H} \sigma$ by

$$
\mathbf{H} \sigma(r, x)=\int_{0}^{x} \sigma(r, s) d s
$$


Suppressing the $x$-variable, the Itô dynamics for the forward rates are thus given by

$$
d r_{t}=\left\{\frac{\partial}{\partial x} r_{t}+\sigma\left(r_{t}\right) \mathbf{H} \sigma\left(r_{t}\right)^{\star}\right\} d t+\sigma\left(r_{t}\right) d W_{t}
$$

and we write this more compactly as

$$
d r_{t}=\mu_{0}\left(r_{t}\right) d t+\sigma\left(r_{t}\right) d W_{t} .
$$

In this way we see clearly how (57) is an SDE on $\mathcal{H}$, specified by its diffusion vector fields $\sigma_{1}, \ldots, \sigma_{m}$ and drift vector field $\mu_{0}$, where $\mu_{0}$ is given by the bracket term in (56). To get some intuition we now formally "divide by $d t$ " and obtain

$$
\frac{d r}{d t}=\mu_{0}\left(r_{t}\right)+\sigma\left(r_{t}\right) \dot{W}_{t},
$$

where the formal time derivative $\dot{W}_{t}$ is interpreted as an "input signal" chosen by chance. As in Section 2.4 we are thus led to study the associated deterministic control system

$$
\frac{d r}{d t}=\mu_{0}\left(r_{t}\right)+\sigma\left(r_{t}\right) u_{t} .
$$

The intuitive idea is now that $\mathcal{G}$ is invariant under (57) if and only if $\mathcal{G}$ is invariant under (59) for all choices of the input signal $u$. It is furthermore geometrically obvious that this happens if and only if the velocity vector $\mu(r)+\sigma(r) u$ is tangential to $\mathcal{G}$ for all points $r \in \mathcal{G}$ and all choices of $u \in R^{m}$. Since the tangent space of $\mathcal{G}$ at a point $G(z)$ is given by $\operatorname{Im}\left[G_{z}^{\prime}(z)\right]$, where $G_{z}^{\prime}$ denotes the Frechet derivative (Jacobian), we are led to conjecture that $\mathcal{G}$ is invariant if and only if the condition

$$
\mu_{0}(r)+\sigma(r) u \in \operatorname{Im}\left[G_{z}^{\prime}(z)\right]
$$

is satisfied for all $u \in R^{m}$. This can also be written

$$
\begin{aligned}
\mu_{0}(r) & \in \operatorname{Im}\left[G_{z}^{\prime}(z)\right], \\
\sigma(r) & \in \operatorname{Im}\left[G_{z}^{\prime}(z)\right],
\end{aligned}
$$

where the last inclusion is interpreted component wise for $\sigma$.

This "result" is, however, not correct due to the fact that the argument above neglects the difference between ordinary calculus, which is used for (59), and Itô calculus, which governs (57). In order to bridge this gap we have to rewrite the analysis in terms of Stratonovich integrals instead of Itô integrals.

Definition 3.5 For given semimartingales $X$ and $Y$, the Stratonovich integral of $X$ with respect to $Y, \int_{0}^{t} X(s) \circ d Y(s)$, is defined as

$$
\int_{0}^{t} X_{s} \circ d Y_{s}=\int_{0}^{t} X_{s} d Y_{s}+\frac{1}{2}\langle X, Y\rangle_{t}
$$


The first term on the RHS is the Itô integral. In the present case, with only Wiener processes as driving noise, we can define the 'quadratic variation process' $\langle X, Y\rangle$ in (60) by

$$
d\langle X, Y\rangle_{t}=d X_{t} d Y_{t}
$$

with the usual 'multiplication rules' $d W \cdot d t=d t \cdot d t=0, d W \cdot d W=d t$. We now recall the main result and raison d'être for the Stratonovich integral.

Proposition 3.2 (Chain rule) Assume that the function $F(t, y)$ is smooth. Then we have

$$
d F\left(t, Y_{t}\right)=\frac{\partial F}{\partial t}\left(t, Y_{t}\right) d t+\frac{\partial F}{\partial y} \circ d Y_{t} .
$$

Thus, in the Stratonovich calculus, the Itô formula takes the form of the standard chain rule of ordinary calculus.

Returning to (57), the Stratonovich dynamics are given by

$$
\begin{aligned}
d r_{t} & =\left\{\frac{\partial}{\partial x} r_{t}+\sigma\left(r_{t}\right) \mathbf{H} \sigma\left(r_{t}\right)^{\star}\right\} d t-\frac{1}{2} d\left\langle\sigma\left(r_{t}\right), W_{t}\right\rangle \\
& +\sigma\left(r_{t}\right) \circ d W_{t} .
\end{aligned}
$$

In order to compute the Stratonovich correction term above we use the infinite dimensional Itô formula (see [16]) to obtain

$$
d \sigma\left(r_{t}\right)=\{\ldots\} d t+\sigma_{r}^{\prime}\left(r_{t}\right) \sigma\left(r_{t}\right) d W_{t},
$$

where $\sigma_{r}^{\prime}$ denotes the Frechet derivative of $\sigma$ w.r.t. the infinite dimensional $r$ variable. From this we immediately obtain

$$
d\left\langle\sigma\left(r_{t}\right), W_{t}\right\rangle=\sigma_{r}^{\prime}\left(r_{t}\right) \sigma\left(r_{t}\right) d t .
$$

Remark 3.3 If the Wiener process $W$ is multidimensional, then $\sigma$ is a vector $\sigma=\left[\sigma_{1}, \ldots, \sigma_{m}\right]$, and the rhs of (65) should be interpreted as

$$
\sigma_{r}^{\prime}\left(r_{t}\right) \sigma\left(r_{t}, x\right)=\sum_{i=1}^{m} \sigma_{i r}^{\prime}\left(r_{t}\right) \sigma_{i}\left(r_{t}\right)
$$

Thus (63) becomes

$$
\begin{aligned}
d r_{t} & =\left\{\frac{\partial}{\partial x} r_{t}+\sigma\left(r_{t}\right) \mathbf{H} \sigma\left(r_{t}\right)^{\star}-\frac{1}{2} \sigma_{r}^{\prime}\left(r_{t}\right) \sigma\left(r_{t}\right)\right\} d t \\
& +\sigma\left(r_{t}\right) \circ d W_{t}
\end{aligned}
$$

We now write (66) as

$$
d r_{t}=\mu\left(r_{t}\right) d t+\sigma\left(r_{t}\right) \circ d W_{t}
$$


where

$$
\mu(r, x)=\frac{\partial}{\partial x} r(x)+\sigma\left(r_{t}, x\right) \int_{0}^{x} \sigma\left(r_{t}, u\right)^{\star} d u-\frac{1}{2}\left[\sigma_{r}^{\prime}\left(r_{t}\right) \sigma\left(r_{t}\right)\right](x) .
$$

For all these arguments to make sense, we need some formal regularity assumptions.

Assumption 3.1 We assume the following .

- For each $i=1, \ldots, m$ the volatility vector field $\sigma_{i}: \mathcal{H} \rightarrow \mathcal{H}$ is smooth.

- The mapping

$$
r \longmapsto \sigma(r) \mathbf{H} \sigma(r)^{\star}-\frac{1}{2} \sigma_{r}^{\prime}(r) \sigma(r)
$$

is a smooth map from $\mathcal{H}$ to $\mathcal{H}$.

- The mapping $z \longmapsto G(z)$ is a smooth embedding, so in particular the Frechet derivative $G_{z}^{\prime}(z)$ is injective for all $z \in \mathcal{Z}$.

Given the heuristics above, our main result is not surprising. The formal proof, which is somewhat technical, is left out. See [5].

Theorem 3.1 (Invariance Theorem) Under Assumption 3.1, the forward curve manifold $\mathcal{G}$ is locally invariant for the forward rate process $r_{t}(x)$ in $\mathcal{M}$ if and only if,

$$
\begin{aligned}
G_{x}^{\prime}(z)+\sigma(r) \mathbf{H} \sigma(r)^{\star}-\frac{1}{2} \sigma_{r}^{\prime}(r) \sigma(r) & \in \operatorname{Im}\left[G_{z}^{\prime}(z)\right], \\
\sigma(r) & \in \operatorname{Im}\left[G_{z}^{\prime}(z)\right],
\end{aligned}
$$

hold for all $z \in \mathcal{Z}$ with $r=G(z)$. The condition (70) is to be interpreted component wise.

Here, $G_{z}^{\prime}$ and $G_{x}^{\prime}$ denote the Frechet derivative of $G$ with respect to $z$ and $x$, respectively. The condition (70) is interpreted component wise for $\sigma$. Condition (69) is called the consistent drift condition, and (70) is called the consistent volatility condition.

Remark 3.4 It is easily seen that if the family $G$ is invariant under shifts in the $x$-variable, then we will automatically have the relation

$$
G_{x}^{\prime}(z) \in \operatorname{Im}\left[G_{z}^{\prime}(z)\right]
$$

so in this case the relation (69) can be replaced by

$$
\sigma(r) \mathbf{H} \sigma(r)^{\star}-\frac{1}{2} \sigma_{r}^{\prime}(r) \sigma(r) \in \operatorname{Im}\left[G_{z}^{\prime}(z)\right]
$$

with $r=G(z)$ as usual. 


\subsection{Examples}

The results above are extremely easy to apply in concrete situations. As a test case we consider the Nelson-Siegel (see [34]) family of forward rate curves. We analyze the consistency of this family with the Ho-Lee and Hull-White interest rate models. It should be emphasized that these examples are chosen only in

order to illustrate the general methodology. For more examples and details, see $[5]$.

\subsubsection{The Nelson-Siegel Family}

The Nelson-Siegel (henceforth NS) forward curve manifold $\mathcal{G}$ is parameterized by $z \in R^{4}$, the curve $x \longmapsto G(z, x)$ as

$$
G(z, x)=z_{1}+z_{2} e^{-z_{4} x}+z_{3} x e^{-z_{4} x} .
$$

For $z_{4} \neq 0$, the Frechet derivatives are easily obtained as

$$
\begin{gathered}
G_{z}^{\prime}(z, x)=\left[1, e^{-z_{4} x}, x e^{-z_{4} x},-\left(z_{2}+z_{3} x\right) x e^{-z_{4} x}\right], \\
G_{x}^{\prime}(z, x)=\left(z_{3}-z_{2} z_{4}-z_{3} z_{4} x\right) e^{-z_{4} x} .
\end{gathered}
$$

In order for the image of this map to be included in $\mathcal{H}_{\gamma}$, we need to impose the condition $z_{4}>-\gamma / 2$. In this case, the natural parameter space is thus $\mathcal{Z}=\left\{z \in R^{4}: z_{4} \neq 0, z_{4}>-\gamma / 2\right\}$. However, as we shall see below, the results are uniform w.r.t. $\gamma$. Note that the mapping $G$ indeed is smooth, and for $z_{4} \neq 0$, $G$ and $G_{z}^{\prime}$ are also injective.

In the degenerate case $z_{4}=0$, we have

$$
G(z, x)=z_{1}+z_{2}+z_{3} x,
$$

We return to this case below.

\subsubsection{The Hull-White and Ho-Lee Models}

As our test case, we analyze the Hull and White (henceforth HW) extension of the Vasiček model. On short rate form the model is given by

$$
d R(t)=\{\Phi(t)-a R(t)\} d t+\sigma d W(t),
$$

where $a, \sigma>0$. As is well known, the corresponding forward rate formulation is

$$
d r_{t}(x)=\beta(t, x) d t+\sigma e^{-a x} d W_{t} .
$$

Thus, the volatility function is given by $\sigma(x)=\sigma e^{-a x}$, and the conditions of Theorem 3.1 become

$$
\begin{aligned}
G_{x}^{\prime}(z, x)+\frac{\sigma^{2}}{a}\left[e^{-a x}-e^{-2 a x}\right] & \in \operatorname{Im}\left[G_{z}^{\prime}(z, x)\right], \\
\sigma e^{-a x} & \in \operatorname{Im}\left[G_{z}^{\prime}(z, x)\right] .
\end{aligned}
$$


To investigate whether the NS manifold is invariant under HW dynamics, we start with (78) and fix a $z$-vector. We then look for constants (possibly depending on $z) A, B, C$, and $D$, such that for all $x \geq 0$ we have

$$
\sigma e^{-a x}=A+B e^{-z_{4} x}+C x e^{-z_{4} x}-D\left(z_{2}+z_{3} x\right) x e^{-z_{4} x} .
$$

This is possible if and only if $z_{4}=a$, and since (78) must hold for all choices of $z \in \mathcal{Z}$ we immediately see that HW is inconsistent with the full NS manifold (see also the Notes below).

Proposition 3.3 (Nelson-Siegel and Hull-White) The Hull-White model is inconsistent with the NS family.

We have thus obtained a negative result for the HW model. The NS manifold is 'too small' for HW, in the sense that if the initial forward rate curve is on the manifold, then the HW dynamics will force the term structure off the manifold within an arbitrarily short period of time. For more positive results see [5].

Remark 3.5 It is an easy exercise to see that the minimal manifold which is consistent with $\mathrm{HW}$ is given by

$$
G(z, x)=z_{1} e^{-a x}+z_{2} e^{-2 a x}
$$

In the same way, one may easily test the consistency between NS and the model obtained by setting $a=0$ in (75). This is the continuous time limit of the Ho and Lee model [27], and is henceforth referred to as HL. Since we have a pedagogical point to make, we give the results on consistency, which are as follows.

\section{Proposition 3.4 (Nelson-Siegel and Ho-Lee)}

(a) The full NS family is inconsistent with the Ho-Lee model.

(b) The degenerate family $G(z, x)=z_{1}+z_{3} x$ is in fact consistent with Ho-Lee.

Remark 3.6 We see that the minimal invariant manifold provides information about the model. From the result above, the HL model is closely tied to the class of affine forward rate curves. Such curves are unrealistic from an economic point of view, implying that the HL model is overly simplistic.

\subsection{The Filipovic State Space Approach to Consistency}

As we very easily detected above, neither the HW nor the HL model is consistent with the Nelson-Siegel family of forward rate curves. A much more difficult problem is to determine whether any interest rate model is. This is Problem II 
in Section 3.1 for the NS family, and in a very general setting, inverse consistency problems like this has been studied in great detail by Filipovic in [19], [20], and [21]. In this section we will give an introduction to the Filipovic state space approach to the (inverse) consistency problem, and we will also study a small laboratory example.

The study will be done within the framework of a factor model.

Definition 3.6 $A$ factor model for the forward rate process $r$ consists of the following objects and relations.

- Ad-dimensional factor or state process $Z$ with $Q$-dynamics of the form

$$
d Z_{t}=a\left(Z_{t}\right) d t+b\left(Z_{t}\right) d W_{t},
$$

where $W$ is an $m$-dimensional Wiener process. We denote by $a_{i}$ the $i: t h$ component of the column vector $a$, and by $b_{i}$ the $i$ : th row of the matrix $b$.

\section{- A smooth output mapping}

$$
G: R^{d} \rightarrow \mathcal{H} .
$$

For each $z \in R^{d}, G(z)$ is thus a real valued $C^{\infty}$ function and it's value at the point $x \in R$ is denoted by $G(z, x)$.

- The forward rate process is then defined by

$$
r_{t}=G\left(Z_{t}\right),
$$

or on component form

$$
r_{t}(x)=G\left(Z_{t}, x\right) .
$$

Since we have given the $Z$ dynamics under the martingale measure $Q$, it is obvious that there has to be some consistency requirements on the relations between $a, b$ and $G$ in order for $r$ in (81) to be a specification of the forward rate process under $Q$. The obvious way of deriving the consistency requirements is to compute the $r$ dynamics from (80)-(81) and then to compare the result with the general form of the forward rate equation in (4). For ease of notation we will use the shorthand notation

$$
G_{x}=\frac{\partial G}{\partial x}, \quad G_{i}=\frac{\partial G}{\partial z_{i}}, \quad G_{i}=\frac{\partial^{2} G}{\partial z_{i} \partial z_{j}}
$$

From the Itô formula, (80), and (81) we obtain

$$
\begin{aligned}
d r_{t} & =\left\{\sum_{i=1}^{d} G_{i}\left(Z_{t}\right) a_{i}\left(Z_{t}\right) d t+\frac{1}{2} \sum_{i, j=1}^{d} G_{i j}\left(Z_{t}\right) b_{i}\left(Z_{t}\right) b_{j}^{\star}\left(Z_{t}\right)\right\} d t \\
& +\sum_{i=1}^{d} G_{i}\left(Z_{t}\right) b_{i}\left(Z_{t}\right) d W_{t}
\end{aligned}
$$


where * denotes transpose. Going back to the forward rate equation (4) we can identify the volatility process as

$$
\sigma_{t}=\sum_{i=1}^{d} G_{i}\left(Z_{t}\right) b_{i}\left(Z_{t}\right) .
$$

We now insert this into the drift part of (4). We then use (81) to deduce that $\mathbf{F} r_{t}=G_{x}\left(Z_{t}\right)$ and also insert this expression into the drift part of (4). Comparing the resulting equation with (84) gives us the required consistency conditions.

Proposition 3.5 (Filipovic) Under a martingale measure $Q$, the following relation must hold identically in $(z, x)$.

$$
\begin{aligned}
& G_{x}(z, x)+\sum_{i, j=1}^{d} b_{i}(z) b_{j}^{\star}(z) G_{i}(z, x) \int_{0}^{x} G_{j}(z, s) d s \\
& =\sum_{i=1}^{d} G_{i}(z, x) a_{i}(z)+\frac{1}{2} \sum_{i, j=1}^{d} G_{i j}(z, x) b_{i}(z) b_{j}^{\star}(z)
\end{aligned}
$$

We can view the consistency equation (86) in three different ways.

- We can check consistency for a given specification of $G, a, b$.

- We can specify $a$ and $b$. Then (86) is a PDE for the determination of a consistent output function $G$.

- We can specify $G$, i.e. we can specify a finite dimensional manifold of forward rate curves, and then use (86) to investigate whether there exist an underlying consistent state vector process $Z$, and if so, to find $a$ and $b$. This inverse problem is precisely Problem II in Section 3.1.

We will focus on the last inverse problem above, and to see how the consistency equation can be used, we now go on to study to two simple laboratory examples.

Example 3.1 In this example we consider the 2-dimensional manifold of linear forward rate curves, i.e. the output function $G$ defined by

$$
G(z, x)=z_{1}+z_{2} x .
$$

This is not a very natural example from a finance point of view, but it is a good illustration of technique. The question we ask is whether there exist some forward rate model consistent with the class of linear forward rate curves and if so what the factor dynamics look like. For simplicity we restrict ourselves to the case of a scalar driving Wiener process, but the reader is invited to analyze the (perhaps more natural) case with a two-dimensional $W$. 
We thus model the factor dynamics as

$$
\begin{aligned}
d Z_{1, t} & =a_{1}\left(Z_{t}\right) d t+b_{1}\left(Z_{t}\right) d W_{t}, \\
d Z_{2, t} & =a_{1}\left(Z_{t}\right) d t+b_{2}\left(Z_{t}\right) d W_{t} .
\end{aligned}
$$

In this case we have

$$
\begin{array}{ll}
G_{x}(z, x)=z_{2}, & G_{1}(z, x)=1, \quad G_{2}(z, x)=x \\
G_{11}(z, x)=0, & G_{12}(z, x)=0, \quad G_{22}(z, x)=0,
\end{array}
$$

and

$$
\int_{0}^{x} G_{1}(z, s) d s=x, \quad \int_{0}^{x} G_{2}(z, s) d s=\frac{1}{2} x^{2},
$$

so the consistency equation (86) becomes

$$
z_{2}+b_{1}^{2}(z) x+b_{1}(z) b_{2}(z) \frac{1}{2} x^{2}+b_{2}(z) b_{1}(z) x^{2}+b_{2}^{2}(z) \frac{1}{2} x^{3}=a_{1}(z)+a_{2}(z) x
$$

Identifying coefficients we see directly that $b_{2}=0$ so the equation reduces to

$$
z_{2}+b_{1}^{2}(z) x=a_{1}(z)+a_{2}(z) x
$$

which gives us the relations $a_{1}=z_{2}$ and $a_{2}=b_{1}^{2}$. Thus we see that for this choice of $G$ there does indeed exist a class of consistent factor models, with factor dynamics given by

$$
\begin{aligned}
d Z_{1, t} & =Z_{2, t} d t+b_{1}\left(Z_{t}\right) d W_{t} \\
d Z_{2, t} & =b_{1}^{2}\left(Z_{t}\right) d t .
\end{aligned}
$$

Here $b_{1}$ can be chosen completely freely (subject only to regularity conditions). Choosing $b_{1}(z)=1$, we see that the factor $Z_{2}$ is essentially running time, and the model is then in fact a special case of the Ho-Lee model.

Example 3.2 We now go on to study the more complicated two-dimensional manifold of exponential forward rate curves, given by the output function

$$
G(z, x)=z_{1} e^{z_{2} x} .
$$

This is a simplified version of the Nelson-Siegel manifold, so it will give us some insight into the consistency problem for the NS case. In this case we will assume two independent driving Wiener processes $W^{1}$, and $W^{2}$, and we will assume factor dynamics of the form

$$
\begin{aligned}
& d Z_{1, t}=a_{1}\left(Z_{t}\right) d t+b_{11}\left(Z_{t}\right) d W_{t}^{1}, \\
& d Z_{2, t}=a_{2}\left(Z_{t}\right) d t+b_{22}\left(Z_{t}\right) d W_{t}^{2} .
\end{aligned}
$$


Note that the factors are being driven by independent Wiener processes. The reader is invited to study the general case when both $W^{1}$ and $W^{2}$ enters into both equations. In our case we have

$$
\begin{aligned}
b_{1}(z) & =\left[b_{11}(z), 0\right], \\
b_{2}(z) & =\left[0, b_{22}(z)\right], \\
G_{x}(z, x) & =z_{1} z_{2} e^{z_{2} x}, \\
G_{1}(z, x) & =e^{z_{2} x} \\
\int_{0}^{x} G_{1}(z, s) d s & =z_{2}^{-1}\left(e^{z_{2} x}-1\right), \\
G_{11}(z, x) & =0 \\
G_{2}(z, x) & =z_{1} x e^{z_{2} x} \\
G_{22}(z, x) & =z_{1} x^{2} e^{z_{2} x} \\
\int_{0}^{x} G_{2}(z, s) d s & =z_{1} z_{2}^{-1} x e^{z_{2} x}-z_{1} z_{2}^{-2} e^{z_{2} x}+z_{2}^{-2} .
\end{aligned}
$$

The consistency equation thus becomes

$$
\begin{aligned}
& z_{1} z_{2} e^{z_{2} x} \\
& +b_{11}^{2}(z) z_{2}^{-1} e^{2 z_{2} x}-b_{11}^{2}(z) z_{2}^{-1} e^{z_{2} x} \\
& +b_{22}^{2}(z) z_{1}^{2} z_{2}^{-1} x^{2} e^{2 z_{2} x}-b_{22}^{2}(z) z_{1}^{2} z_{2}^{-2} x e^{2 z_{2} x}+b_{22}^{2}(z) z_{1} z_{2}^{-2} x e^{z_{2} x} \\
& =a_{1}(z) e^{z_{2} x}+a_{2}(z) z_{1} x e^{z_{2} x}+\frac{1}{2} b_{22}^{2}(z) z_{1} x^{2} e^{z_{2} x}
\end{aligned}
$$

Rearranging terms we have

$$
\begin{aligned}
& e^{z_{2} x}\left[z_{1} z_{2}+b_{11}(z) z_{2}^{-1}-a_{1}(z)\right] \\
+ & x e^{z_{2} x}\left[b_{22}^{2}(z) z_{1} z_{2}^{-2}-a_{2}(z) z_{1}\right] \\
+ & x^{2} e^{z_{2} x}\left[-\frac{1}{2} b_{22}^{2}(z) z_{1}\right] \\
+ & e^{2 z_{2} x}\left[b_{11}^{2}(z) z_{2}^{-1}\right] \\
+ & x e^{2 z_{2} x}\left[-b_{22}^{2}(z) z_{1}^{2} z_{2}^{-2}\right] \\
+ & x^{2} e^{2 z_{2} x}\left[b_{22}^{2}(z) z_{1}^{2} z_{2}^{-1}\right] \\
= & 0
\end{aligned}
$$

From the linear independence of the quasi-exponential functions, we immediately obtain

$$
\begin{aligned}
b_{11}(z) & =0, \quad b_{22}(z)=0, \\
a_{2}(z) & =0, \quad a_{1}(z)=z_{1} z_{2} .
\end{aligned}
$$

The only consistent $Z$-dynamics are thus given by

$$
\begin{aligned}
d Z_{1, t} & =z_{2, t} Z_{1, t}, d t \\
d Z_{2, t} & =0
\end{aligned}
$$


which implies that $Z_{2}$ is constant and that

$$
Z_{1, t}=Z_{1,0} e^{Z_{2} t} .
$$

We thus see that, apart from allowing randomness in the initial values, both $Z_{1}$ and $Z_{2}$ evolve along deterministic paths, where in fact $Z_{2}$ stays constant whereas $Z_{1}$ grows exponentially at the rate $Z_{2}$. In other words, there exists no non-trivial factor model which is consistent with the class of exponential forward rate curves.

As we have seen, the calculations quickly becomes rather messy, and it is thus a formidable task to find the set of consistent factor models for a more complicated manifold like, say, the Nelson-Siegel family of forward rate curves. Since the NS family is four-dimensional we would need a four dimensional factor model with four independent Wiener processes (all of which would be driving each of the four equations).

In [19] the case of the NS family was indeed studied, and it was proved that no nontrivial Wiener driven model is consistent with NS. Thus, for a model to be consistent with Nelson-Siegel, it must be deterministic (apart from randomness in the initial conditions). In [20] (which is a technical tour de force) this result was then extended to a much larger exponential polynomial family than the NS family.

\section{7 $\quad$ Notes}

The section is largely based on [5] and [19]. In our presentation we have used strong solutions of the infinite dimensional forward rate SDE. This is of course restrictive. The invariance problem for weak solutions has been studied by Filipovic in [22] and [21]. An alternative way of studying invariance is by using some version of the Stroock-Varadhan support theorem, and this line of thought is carried out in depth in [38].

\section{The General FDR Problem}

We now turn to Problem 2 in Section 1.3, i.e. the problem when a given forward rate model has a finite dimensional factor realization. For ease of exposition we mostly confine ourselves to time invariant forward rate dynamics. Time varying systems can be treated similarly (see [9]). We will use some ideas and concepts from differential geometry, and a general reference here is [37]. The section is based on [9]. 


\subsection{Setup}

We consider a given volatility structure $\sigma: \mathcal{H} \rightarrow \mathcal{H}^{m}$ and study the induced forward rate model (on Stratonovich form)

$$
d r_{t}=\mu\left(r_{t}\right) d t+\sigma\left(r_{t}\right) \circ d W_{t}
$$

where as before (see Section 3.4).

$$
\mu(r)=\frac{\partial}{\partial x} r+\sigma(r) \mathbf{H} \sigma(r)^{\star}-\frac{1}{2} \sigma_{r}^{\prime}(r) \sigma(r)
$$

Throughout the rest of the section, Assumption 3.1 is in force.

\subsection{The Geometric problem}

Given a specification of the volatility mapping $\sigma$, and an initial forward rate curve $r^{o}$ we now investigate when (and how) the corresponding forward rate process possesses a finite, dimensional realization. We are thus looking for smooth $d$-dimensional vector fields $a$ and $b$, an initial point $z_{0} \in R^{d}$, and a mapping $G: R^{d} \rightarrow \mathcal{H}$ such that $r$, locally in time, has the representation

$$
\begin{aligned}
d Z_{t} & =a\left(Z_{t}\right) d t+b\left(Z_{t}\right) d W_{t}, Z_{0}=z_{0} \\
r_{t}(x) & =G\left(Z_{t}, x\right) .
\end{aligned}
$$

Remark 4.1 Let us clarify some points. Firstly, note that in principle it may well happen that, given a specification of $\sigma$, the $r$-model has a finite dimensional realization given a particular initial forward rate curve $r^{o}$, while being infinite dimensional for all other initial forward rate curves in a neighborhood of $r^{o}$. We say that such a model is a non-generic or accidental finite dimensional model. If, on the other hand, $r$ has a finite dimensional realization for all initial points in a neighborhood of $r^{o}$, then we say that the model is a generically finite dimensional model. In this text we are solely concerned with the generic problem. Secondly, let us emphasize that we are looking for local (in time) realizations.

We can now connect the realization problem to our studies of invariant manifolds.

Proposition 4.1 The forward rate process possesses a finite dimensional realization if and only if there exists an invariant finite dimensional submanifold $\mathcal{G}$ with $r^{\circ} \in \mathcal{G}$.

Proof. See [5] for the full proof. The intuitive argument runs as follows. Suppose that there exists a finite dimensional invariant manifold $\mathcal{G}$ with $r^{o} \in \mathcal{G}$. Then 
$\mathcal{G}$ has a local coordinate system, and we may define the $Z$ process as the local coordinate process for the $r$-process. On the other hand it is clear that if $r$ has a finite dimensional realization as in (110)-(111), then every forward rate curve that will be produced by the model is of the form $x \longmapsto G(z, x)$ for some choice of $z$. Thus there exists a finite dimensional invariant submanifold $\mathcal{G}$ containing the initial forward rate curve $r^{o}$, namely $\mathcal{G}=\operatorname{Im} G$.

Using Theorem 3.1 we immediately obtain the following geometric characterization of the existence of a finite realization.

Corollary 4.1 The forward rate process possesses a finite dimensional realization if and only if there exists a finite dimensional manifold $\mathcal{G}$ containing $r^{o}$, such that, for each $r \in \mathcal{G}$ the following conditions hold.

$$
\begin{aligned}
& \mu(r) \in T_{\mathcal{G}}(r), \\
& \sigma(r) \in T_{\mathcal{G}}(r) .
\end{aligned}
$$

Here $T_{\mathcal{G}}(r)$ denotes the tangent space to $\mathcal{G}$ at the point $r$, and the vector fields $\mu$ and $\sigma$ are as above. The tangency condition for $\sigma$ is as usual interpreted component wise.

\subsection{The Main Result}

Given the volatility vector fields $\sigma_{1}, \ldots, \sigma_{m}$, and hence also the field $\mu$, we now are faced with the problem of determining if there exists a finite dimensional manifold $\mathcal{G}$ with the property that $\mu$ and $\sigma_{1}, \ldots, \sigma_{m}$ are tangential to $\mathcal{G}$ at each point of $\mathcal{G}$. In the case when the underlying space is finite dimensional, this is a standard problem in differential geometry, and we will now give the heuristics.

To get some intuition we start with a simpler problem and therefore consider the space $\mathcal{H}$ (or any other Hilbert space), and a smooth vector field $f$ on the space. For each fixed point $r^{o} \in \mathcal{H}$ we now ask if there exists a finite dimensional manifold $\mathcal{G}$ with $r^{o} \in \mathcal{G}$ such that $f$ is tangential to $\mathcal{G}$ at every point. The answer to this question is yes, and the manifold can in fact be chosen to be one-dimensional. To see this, consider the infinite dimensional ODE

$$
\begin{aligned}
\frac{d r_{t}}{d t} & =f\left(r_{t}\right), \\
r_{0} & =r^{o} .
\end{aligned}
$$

If $r_{t}$ is the solution, at time $t$, of this ODE, we use the notation

$$
r_{t}=e^{f t} r^{o} .
$$

We have thus defined a group of operators $\left\{e^{f t}: t \in R\right\}$, and we note that the set $\left\{e^{f t} r^{o}: t \in R\right\} \subseteq \mathcal{H}$ is nothing else than the integral curve of the vector field 
$f$, passing through $r^{o}$. If we define $\mathcal{G}$ as this integral curve, then our problem is solved, since $f$ will be tangential to $\mathcal{G}$ by construction.

Let us now take two vector fields $f_{1}$ and $f_{2}$ as given, where the reader informally can think of $f_{1}$ as $\sigma$ (in the case of a scalar Wiener process) and $f_{2}$ as $\mu$. We also fix an initial point $r^{o} \in \mathcal{H}$ and the question is if there exists a finite dimensional manifold $\mathcal{G}$, containing $r^{o}$, with the property that $f_{1}$ and $f_{2}$ are both tangential to $\mathcal{G}$ at each point of $\mathcal{G}$. We call such a manifold an tangential manifold for the vector fields. At a first glance it would seem that there always exists an tangential manifold, and that it can even be chosen to be two-dimensional. The geometric idea is that we start at $r^{o}$ and let $f_{1}$ generate the integral curve $\left\{e^{f_{1} s} r^{o}: s \geq 0\right\}$. For each point $e^{f_{1} s} r^{o}$ on this curve we now let $f_{2}$ generate the integral curve starting at that point. This gives us the object $e^{f_{2} t} e^{f_{1} s} r^{o}$ and thus it seems that we sweep out a two dimensional surface $\mathcal{G}$ in $\mathcal{H}$. This is our obvious candidate for an tangential manifold.

In the general case this idea will, however, not work, and the basic problem is as follows. In the construction above we started with the integral curve generated by $f_{1}$ and then applied $f_{2}$, and there is of course no guarantee that we will obtain the same surface if we start with $f_{2}$ and then apply $f_{1}$. We thus have some sort of commutativity problem, and the key concept is the Lie bracket.

Definition 4.1 Given smooth vector fields $f$ and $g$ on $\mathcal{H}$, the Lie bracket $[f, g]$ is a new vector field defined by

$$
[f, g](r)=f^{\prime}(r) g(r)-g^{\prime}(r) f(r)
$$

The Lie bracket measures the lack of commutativity on the infinitesimal scale in our geometric program above, and for the procedure to work we need a condition which says that the lack of commutativity is "small". It turns out that the relevant condition is that the Lie bracket should be in the linear hull of the vector fields.

Definition 4.2 Let $f_{1}, \ldots, f_{n}$ be smooth independent vector fields on some space $X$. Such a system is called a distribution, and the distribution is said to be involutive if

$$
\left[f_{i}, f_{j}\right](x) \in \operatorname{span}\left\{f_{1}(x), \ldots, f_{n}(x)\right\}, \quad \forall i, j,
$$

where the span is the linear hull over the real numbers.

We now have the following basic result, which extends a classic result from finite dimensional differential geometry (see [37]).

Theorem 4.1 (Frobenius) Let $f_{1}, \ldots, f_{k}$ and be independent smooth vector fields in $\mathcal{H}$ and consider a fixed point $r^{o} \in \mathcal{H}$. Then the following statements are equivalent. 
- For each point $r$ in a neighborhood of $r^{o}$, there exists a $k$-dimensional tangential manifold passing through $r$.

- The system $f_{1}, \ldots, f_{k}$ of vector fields is involutive.

Proof. See [9], which provides a self contained proof of the Frobenius Theorem in Banach space.

Let us now go back to our interest rate model. We are thus given the vector fields $\mu, \sigma$, and an initial point $r^{o}$, and the problem is whether there exists a finite dimensional tangential manifold containing $r^{o}$. Using the infinite dimensional Frobenius theorem, this situation is now easily analyzed. Suppose for simplicity that $m=1$ i.e. that we only have one scalar driving Wiener process. Now; if $\{\mu, \sigma\}$ is involutive then there exists a two dimensional tangential manifold. If $\{\mu, \sigma\}$ is not involutive, this means that the Lie bracket $[\mu, \sigma]$ is not in the linear span of $\mu$ and $\sigma$, so then we consider the system $\{\mu, \sigma,[\mu, \sigma]\}$. If this system is involutive there exists a three dimensional tangential manifold. If it is not involutive at least one of the brackets $[\mu,[\mu, \sigma]],[\sigma,[\mu, \sigma]]$ is not in the span of $\{\mu, \sigma,[\mu, \sigma]\}$, and we then adjoin this (these) bracket(s). We continue in this way, forming brackets of brackets, and adjoining these to the linear hull of the previously obtained vector fields, until the point when the system of vector fields thus obtained actually is closed under the Lie bracket operation.

Definition 4.3 Take the vector fields $f_{1}, \ldots, f_{k}$ as given. The Lie algebra generated by $f_{1}, \ldots, f_{k}$ is the smallest linear space (over $R$ ) of vector fields which contains $f_{1}, \ldots, f_{k}$ and is closed under the Lie bracket. This Lie algebra is denoted by

$$
\mathcal{L}=\left\{f_{1}, \ldots, f_{k}\right\}_{L A}
$$

The dimension of $\mathcal{L}$ is defined, for each point $r \in \mathcal{H}$ as

$$
\operatorname{dim}[\mathcal{L}(r)]=\operatorname{dim} \operatorname{span}\left\{f_{1}(r), \ldots, f_{k}(r)\right\} .
$$

Putting all these results together, we can now state the main result on finite dimensional realizations. As can be seen from the arguments above, the fact that we have been studying the particular case of the forward rate equation is not at all essential: all results will continue to hold for any SDE with smooth drift and diffusion vector fields, evolving on a Hilbert space. We therefore state the main realization theorem for an arbitrary SDE in Hilbert space.

Theorem 4.2 (Main Result) Consider the following Stratonovich SDE, evolving in a given Hilbert space $\mathcal{H}$.

$$
d r=\mu\left(r_{t}\right) d t+\sigma\left(r_{t}\right) \circ d W_{t} .
$$


We assume that the drift and diffusion terms $\mu$ and $\sigma$ are smooth vector fields on $\mathcal{H}$.

Then the SDE (115) generically admits a finite dimensional realization if and only if

$$
\operatorname{dim}\left\{\mu, \sigma_{1}, \ldots, \sigma_{m}\right\}_{L A}<\infty
$$

in a neighborhood of $r^{o}$.

The result above thus provides a general solution to Problem 2 from Section 1.3. For any given specification of forward rate volatilities, the Lie algebra can in principle be computed, and the dimension can be checked. Note, however, that the theorem is a pure existence result. If, for example, the Lie algebra has dimension five, then we know that there exists a five-dimensional realization, but the theorem does not directly tell us how to construct a concrete realization. This is the subject of Section 5 below. Note also that realizations are not unique, since any diffeomorphic mapping of the factor space $R^{d}$ onto itself will give a new equivalent realization.

When computing the Lie algebra generated by $\mu$ and $\sigma$, the following observations are often useful.

Lemma 4.1 Take the vector fields $f_{1}, \ldots, f_{k}$ as given. The Lie algebra $\mathcal{L}=$ $\left\{f_{1}, \ldots, f_{k}\right\}_{L A}$ remains unchanged under the following operations.

- The vector field $f_{i}(r)$ may be replaced by $\alpha(r) f_{i}(r)$, where $\alpha$ is any smooth nonzero scalar field.

- The vector field $f_{i}(r)$ may be replaced by

$$
f_{i}(r)+\sum_{j \neq i} \alpha_{j}(r) f_{j}(r)
$$

where $\alpha_{j}$ is any smooth scalar field.

Proof. The first point is geometrically obvious, since multiplication by a scalar field will only change the length of the vector field $f_{i}$, and not its direction, and thus not the tangential manifold. Formally it follows from the "Leibnitz rule" $[f, \alpha g]=\alpha[f, g]-\left(\alpha^{\prime} f\right) g$. The second point follows from the bilinear property of the Lie bracket together with the fact that $[f, f]=0$.

\subsection{Constructing the invariant manifold}

As we have seen above, there exists generically an FDR for (115) if and only if there exists, for any initial point near $r^{o}$ an invariant manifold containing the 
initial point, and this manifold will also be a tangential manifold for all vector fields in the Lie algebra $\{\mu, \sigma\}_{L A}$. In this section we provide a concrete parameterization of this invariant manifold. This result will be used in connection with the construction problem treated in Section 5.

Proposition 4.2 Consider the SDE (115), and assume that the Lie algebra $\mathcal{L}=\{\mu, \sigma\}_{L A}$ is finite dimensional near $r^{o}$. Assume furthermore that we have chosen an involutive system of independent vector fields $f_{1}, \ldots, f_{n}$ such that $\mathcal{L}=\operatorname{span}\left\{f_{1}, \ldots, f_{n}\right\}$. Now choose an initial point $r_{0} \in \mathcal{H}$ near $r^{o}$. Denote the induced invariant (and thus tangential) manifold through $r_{0}$, by $\mathcal{G}$. Define the mapping $G: R^{n} \rightarrow X$ by

$$
G\left(z_{1}, \ldots z_{n}\right)=e^{f_{n} z_{n}} \ldots e^{f_{1} z_{1}} r_{0} .
$$

Then $G$ is a local parameterization of $\mathcal{G}$. Furthermore, the inverse of $G$ restricted to $V$ is a local coordinate system for $\mathcal{G}$ at $r_{0}$.

Proof. It follows directly from the definition of an tangential manifold that $G(z) \in \mathcal{G}$ for all $z$ near 0 in $R^{n}$. Furthermore it is easy to see that $G^{\prime}(0) h=$ $\sum_{i=1}^{n} h_{i} f_{i}\left(x_{0}\right)$ and , since $f_{1}, \ldots, f_{n}$ are independent, $G^{\prime}(0)$ is injective. The inverse function theorem does the rest.

With this machinery we can also very easily solve a related question. Consider a fixed interest rate model, specified by the volatility $\sigma$ and also a fixed family of forward rate curves parameterized by the mapping $G_{0}: R^{k} \rightarrow \mathcal{H}$. Now, if $\mathcal{G}_{0}=\operatorname{Im}\left[G_{0}\right]$ is invariant, then the interest rate model will, given any initial point $r^{o}$ in $\mathcal{G}_{0}$, only produce forward rate curves belonging to $\mathcal{G}_{0}$ or, in the terminology of Section 3, the given model and the family $\mathcal{G}_{0}$ are consistent. If the family is not consistent, then an initial forward rate curve in $\mathcal{G}_{0}$ may produce future forward rate curves outside $\mathcal{G}_{0}$, and the question arises how to construct the smallest possible family of forward rate curves which contains the initial family $\mathcal{G}_{0}$, and is consistent (i.e. invariant) w.r.t the interest rate model. As a concrete example, one may want to find the minimal extension of the Nelson-Siegel family of forward rate curves (see [34], [5]) which is consistent with the Hull-White (extended Vasiček) model. In particular one would like to know under what conditions this minimal extension of $\mathcal{G}_{0}$ is finite dimensional.

In geometrical terms we thus want to construct the minimal manifold containing $\mathcal{G}_{0}$, which is tangential w.r.t. the vector fields $\mu, \sigma_{1}, \ldots, \sigma_{m}$. The solution is obvious: For very point on $\mathcal{G}_{0}$ we construct the minimal tangential manifold through that point, and then we define the extension $\mathcal{G}$ as the union of all these fibers. Thus we have the following result, the proof of which is obvious. Concrete applications will be given below. 
Proposition 4.3 Consider a fixed volatility mapping $\sigma$, and let $\mathcal{G}_{0}$ be a $k$ dimensional submanifold parameterized by $G_{0}: R^{k} \rightarrow \mathcal{H}$. Then $\mathcal{G}_{0}$ can be extended to a finite dimensional invariant submanifold $\mathcal{G}$, if and only if

$$
\operatorname{dim}\left\{\mu, \sigma_{1}, \ldots, \sigma_{m}\right\}_{L A}<\infty .
$$

Moreover, if $\mathcal{G}_{0}$ is transversal to $\{\mu, \sigma\}_{L A}$ and if the Lie algebra is spanned by the independent vector fields $f_{1}, \ldots, f_{d}$, then $\operatorname{dim} \mathcal{G}=k+d$ and a parameterization of $\mathcal{G}$ is given by the map $G: R^{k+d} \rightarrow \mathcal{H}$, defined by

$$
G\left(z_{1}, \ldots, z_{k}, y_{1}, \ldots, y_{d}\right)=e^{f_{d} y_{d}} \ldots e^{f_{1} y_{1}} G_{0}\left(z_{1}, \ldots, z_{k}\right) .
$$

Remark 4.2 The term "transversal" above means that no vector in the Lie algebra $\mathcal{L}(\mu, \sigma)$ is contained the tangent space of $\mathcal{G}_{0}$ at any point of $\mathcal{G}_{0}$. This prohibits an integral curve of $\mathcal{L}$ to be contained in $\mathcal{G}_{0}$, which otherwise would lead to an extension with lower dimension than $d+k$. In such a case the parameterization above would amount to an over parameterization in the sense that $G$ would not be injective.

\subsection{Applications}

In this section we give some simple applications of the theory developed above. For more examples and results, see [9].

\subsubsection{Constant Volatility: Existence of FDRs}

We start with the simplest case, which is when the volatility $\sigma(r, x)$ does not depend on $r$. In other words, $\sigma$ is of the form $\sigma(r, x)=\sigma(x)$, and $\sigma$ is thus a constant vector field on $\mathcal{H}$. We assume for brevity of notation that we have only one driving Wiener process. Since $\sigma$ is deterministic we have no Stratonovich correction term and the vector fields are given by

$$
\begin{aligned}
\mu(r, x) & =\mathbf{F} r(x)+\sigma(x) \int_{0}^{x} \sigma^{\star}(s) d s, \\
\sigma(r, x) & =\sigma(x),
\end{aligned}
$$

where as before $\mathbf{F}=\frac{\partial}{\partial x}$.

The Frechet derivatives are trivial in this case. Since $\mathbf{F}$ is linear (and bounded in our space), and $\sigma$ is constant as a function of $r$, we obtain

$$
\begin{aligned}
& \mu_{r}^{\prime}=\mathbf{F}, \\
& \sigma_{r}^{\prime}=0 .
\end{aligned}
$$

Thus the Lie bracket $[\mu, \sigma]$ is given by

$$
[\mu, \sigma]=\mathbf{F} \sigma,
$$


and in the same way we have

$$
[\mu,[\mu, \sigma]]=\mathbf{F}^{2} \sigma .
$$

Continuing in the same manner it is easily seen that the relevant Lie algebra $\mathcal{L}$ is given by

$$
\mathcal{L}=\{\mu, \sigma\}_{L A}=\operatorname{span}\left\{\mu, \sigma, \mathbf{F} \sigma, \mathbf{F}^{2} \sigma, \ldots\right\}=\operatorname{span}\left\{\mu, \mathbf{F}^{n} \sigma ; n=0,1,2, \ldots\right\}
$$

and it is thus clear that $\mathcal{L}$ is finite dimensional (at each point $r$ ) if and only if the function space

$$
\operatorname{span}\left\{\mathbf{F}^{n} \sigma ; n=0,1,2, \ldots\right\}
$$

is finite dimensional. This, on the other hand, occurs if and only if each component of $\sigma$ solves a linear ODE with constant coefficients. This argument is easily extended to the case of a multidimensional driving Wiener process so, using Lemma 2.1, we can finally state the existence result for constant volatility models.

Proposition 4.4 Assume that the volatility components $\sigma_{1}, \ldots, \sigma_{m}$ are deterministic, i.e. of the form

$$
\sigma_{i}(r, x)=\sigma_{i}(x), \quad i=1, \ldots, m .
$$

Then there exists a finite dimensional realization if and only if the function space

$$
\operatorname{span}\left\{\mathbf{F}^{n} \sigma_{i} ; i=1, \ldots, m ; n=0,1,2, \ldots\right\}
$$

is finite dimensional. This occurs if and only if each component of $\sigma$ is a quasiexponential function.

\subsubsection{Constant Volatility: Invariant Manifolds}

For models with constant volatility vector fields, we now turn to the construction of invariant manifolds, and to this end we assume that the Lie algebra above is finite dimensional. Thus it is spanned by a finite number of vector fields as

$$
\{\mu, \sigma\}_{L A}=\operatorname{span}\left\{\mu, \sigma_{i}^{(k)} ; i=1, \ldots, m ; k=0,1, \ldots, n_{i}\right\},
$$

where

$$
\sigma_{i}^{(k)}(x)=\frac{\partial^{k} \sigma_{i}}{\partial x^{k}}(x)
$$

In order to apply Proposition 4.2 and Proposition 4.3, we have to compute the operators $\exp [\mu t]$ and $\exp \left[\sigma_{i}^{(k)} t\right]$, i.e. we have to solve $\mathcal{H}$-valued ODEs. We recall that

$$
\mu(r)=\mathbf{F} r+D,
$$


where the constant field $D$ is given

$$
D(x)=\sum_{i}^{m} \sigma_{i}(x) \int_{0}^{x} \sigma_{i}^{\star}(s) d s,
$$

which can be written as

$$
D(x)=\frac{1}{2} \frac{\partial}{\partial x}\|S(x)\|^{2},
$$

where $S(x)=\int_{0}^{x} \sigma(s) d s$. Thus $e^{\mu t}$ is obtained by solving

$$
\frac{d r}{d t}=\mathbf{F} r+D
$$

This is a linear equation, and from Proposition 2.1 we obtain the solution

$$
r_{t}=e^{\mathbf{F} t} r_{0}+\int_{0}^{t} e^{\mathbf{F}(t-s)} D d s
$$

so

$$
\left(e^{\mu t} r_{0}\right)(x)=r_{0}(x+t)+\frac{1}{2}\left(\|S(x+t)\|^{2}-\|S(x)\|^{2}\right) .
$$

The vector fields $\sigma_{i}^{(k)}$ are constant, so the corresponding ODEs are trivial. We have

$$
e^{\sigma_{i}^{(k)}} t_{0}=r_{0}+\sigma_{i}^{(k)} t .
$$

We thus have the following results on the parameterization of invariant manifolds. For a given mapping $G: R^{n} \rightarrow \mathcal{H}$, we write $G(z)(x)$ or $G(z, x)$ to denote the function $G(z) \in \mathcal{H}$ evaluated at $x \in R_{+}$.

Proposition 4.5 The invariant manifold generated by the initial forward rate curve $r_{0}$ is parameterized as

$$
\begin{aligned}
& G\left(z_{0}, z_{i}^{k} ; i=1, \ldots, m ; k=0, \ldots, n_{i}\right)(x) \\
= & r_{0}\left(x+z_{0}\right)+\frac{1}{2}\left(\|S(x+t)\|^{2}-\|S(x)\|^{2}\right)+\sum_{i=1}^{m} \sum_{k=0}^{n_{i}} \sigma_{i}^{(k)}(x) z_{i}^{k} .
\end{aligned}
$$

If the $k$-dimensional manifold $\mathcal{G}_{0}$ is transversal to $\mathcal{L}\{\mu, \sigma\}$ and parameterized by $G_{0}\left(y_{1}, \ldots, y_{k}\right)$, then the minimal consistent (i.e. invariant) extension is parameterized as

$$
\begin{aligned}
& G\left(y_{1}, \ldots, y_{k}, z_{0}, z_{i}^{k} ; i=1, \ldots, m ; k=0, \ldots, n_{i}\right)(x) \\
= & G_{0}\left(y_{1}, \ldots, y_{k}\right)\left(x+z_{0}\right)+\frac{1}{2}\left(\left\|S\left(x+z_{0}\right)\right\|^{2}-\|S(x)\|^{2}\right)+\sum_{i=1}^{m} \sum_{k=0}^{n_{i}} \sigma_{i}^{(k)}(x) z_{i}^{k} .
\end{aligned}
$$


Note that if $\mathcal{G}_{0}$ is invariant under shift in the $x$-variable (this is in fact the typical case), then a simpler parameterization of $\mathcal{G}$ is given by

$$
\begin{aligned}
& G\left(y_{1}, \ldots, y_{k}, z_{0}, z_{i}^{k} ; i=1, \ldots, m ; k=0, \ldots, n_{i}\right)(x) \\
= & G_{0}\left(y_{1}, \ldots, y_{k}\right)(x)+\frac{1}{2}\left(\left\|S\left(x+z_{0}\right)\right\|^{2}-\|S(x)\|^{2}\right)+\sum_{i=1}^{m} \sum_{k=0}^{n_{i}} \sigma_{i}^{(k)}(x) z_{i}^{k} .
\end{aligned}
$$

As a concrete application let us consider the simple case when $m=1$ and

$$
\sigma(x)=\sigma e^{-a x},
$$

where, with a slight abuse of notation, $a$ and $\sigma$ denote positive constants. As is well known, this is the HJM formulation of the Hull-White extension of the Vasiček model [28],[36]. In this case we have

$$
S(x)=\frac{\sigma}{a}\left[1-e^{-a x}\right] .
$$

The relevant function space

$$
\left\{\mathbf{F}^{n} \sigma ; n \geq 0\right\}=\left\{\frac{\partial^{n}}{\partial x^{n}} e^{-a x} ; n \geq 0\right\}
$$

is obviously one-dimensional and spanned by the single function $e^{-a x}$, so the Lie algebra is two-dimensional.

As the given manifold $\mathcal{G}_{0}$ we take the Nelson-Siegel ([34]) family of forward rate curves, parameterized as

$$
G_{0}\left(y_{1}, \ldots, y_{4}\right)(x)=y_{1}+y_{2} e^{-y_{4} x}+y_{3} x e^{-y_{4} x} .
$$

This family is obviously invariant under shift in $x$, so we have the following result.

Proposition 4.6 For a given initial forward rate curve $r_{0}$, the invariant manifold generated by the Hull-White extended Vasiček model is parameterized by

$G\left(z_{0}, z_{1}\right)(x)=r_{0}\left(x+z_{0}\right)+e^{-a x} \frac{\sigma^{2}}{a^{2}}\left[1-e^{-a z_{0}}\right]-e^{-2 a x} \frac{\sigma^{2}}{2 a^{2}}\left[1-e^{-2 a z_{0}}\right]+z_{1} e^{-a x}$.

The minimal extension of the NS family consistent with the Hull-White extended Vasiček model is parameterized by

$$
\begin{aligned}
& G\left(z_{0}, z_{1}, y_{1}, \ldots, y_{4}\right)(x)=y_{1}+y_{2} e^{-y_{4} x}+y_{3} x e^{-y_{4} x} \\
& +e^{-a x} \frac{\sigma^{2}}{a^{2}}\left[1-e^{-a z_{0}}\right]-e^{-2 a x} \frac{\sigma^{2}}{2 a^{2}}\left[1-e^{-2 a z_{0}}\right]+z_{1} e^{-a x} .
\end{aligned}
$$




\subsubsection{Constant Direction Volatility}

We go on to study the most natural extension of the deterministic volatility case namely the case when the volatility is of the form

$$
\sigma(r, x)=\varphi(r) \lambda(x) .
$$

We restrict ourselves to the case of a scalar Wiener process. In this case the individual vector field $\sigma$ has the constant direction $\lambda \in \mathcal{H}$, but is of varying length, determined by $\varphi$, where $\varphi$ is allowed to be any smooth functional of the entire forward rate curve. In order to avoid trivialities we make the following assumption.

Assumption 4.1 We assume that $\varphi(r) \neq 0$ for all $r \in \mathcal{H}$.

After a simple calculation the drift vector $\mu$ turns out to be

$$
\mu(r)=\mathbf{F} r+\varphi^{2}(r) D-\frac{1}{2} \varphi^{\prime}(r)[\lambda] \varphi(r) \lambda,
$$

where $\varphi^{\prime}(r)[\lambda]$ denotes the Frechet derivative $\varphi^{\prime}(r)$ acting on the vector $\lambda$, and where the constant vector $D \in \mathcal{H}$ is given by

$$
D(x)=\lambda(x) \int_{0}^{x} \lambda(s) d s .
$$

We now want to know under what conditions on $\varphi$ and $\lambda$ we have a finite dimensional realization, i.e. when the Lie algebra generated by

$$
\begin{aligned}
& \mu(r)=\mathbf{F} r+\varphi^{2}(r) D-\frac{1}{2} \varphi^{\prime}(r)[\lambda] \varphi(r) \lambda, \\
& \sigma(r)=\varphi(r) \lambda,
\end{aligned}
$$

is finite dimensional. Under Assumption 4.1 we can use Lemma 4.1, to see that the Lie algebra is in fact generated by the simpler system of vector fields

$$
\begin{aligned}
& f_{0}(r)=\mathbf{F} r+\Phi(r) D, \\
& f_{1}(r)=\lambda,
\end{aligned}
$$

where we have used the notation

$$
\Phi(r)=\varphi^{2}(r) .
$$

Since the field $f_{1}$ is constant, it has zero Frechet derivative. Thus the first Lie bracket is easily computed as

$$
\left[f_{0}, f_{1}\right](r)=\mathbf{F} \lambda+\Phi^{\prime}(r)[\lambda] D .
$$

The next bracket to compute is $\left[\left[f_{0}, f_{1}\right], f_{1}\right]$ which is given by

$$
\left[\left[f_{0}, f_{1}\right], f_{1}\right]=\Phi^{\prime \prime}(r)[\lambda ; \lambda] D .
$$


Note that $\Phi^{\prime \prime}(r)[\lambda ; \lambda]$ is the second order Frechet derivative of $\Phi$ operating on the vector pair $[\lambda ; \lambda]$. This pair is to be distinguished from (notice the semicolon) the Lie bracket $[\lambda, \lambda]$ (with a comma), which if course would be equal to zero. We now make a further assumption.

Assumption 4.2 We assume that $\Phi^{\prime \prime}(r)[\lambda ; \lambda] \neq 0$ for all $r \in \mathcal{H}$.

Given this assumption we may again use Lemma 4.1 to see that the Lie algebra is generated by the following vector fields

$$
\begin{aligned}
& f_{0}(r)=\mathbf{F} r, \\
& f_{1}(r)=\lambda, \\
& f_{3}(r)=\mathbf{F} \lambda, \\
& f_{4}(r)=D .
\end{aligned}
$$

Of these vector fields, all but $f_{0}$ are constant, so all brackets are easy. After elementary calculations we see that in fact

$$
\{\mu, \sigma\}_{L A}=\operatorname{span}\left\{\mathbf{F} r, \mathbf{F}^{n} \lambda, \mathbf{F}^{n} D ; n=0,1, \ldots\right\} .
$$

From this expression it follows immediately that a necessary condition for the Lie algebra to be finite dimensional is that the vector space spanned by $\left\{\mathbf{F}^{n} \lambda ; n \geq 0\right\}$ is finite dimensional. This occurs if and only if $\lambda$ is quasi-exponential (see Remark 2.2). If, on the other hand, $\lambda$ is quasi-exponential, then we know from Lemma 2.1, that also $D$ is quasi-exponential, since it is the integral of the QE function $\lambda$ multiplied by the QE function $\lambda$. Thus the space $\left\{\mathbf{F}^{n} D ; n=0,1, \ldots\right\}$ is also finite dimensional, and we have proved the following result.

Proposition 4.7 Under Assumptions 4.1 and 4.2, the interest rate model with volatility given by $\sigma(r, x)=\varphi(r) \lambda(x)$ has a finite dimensional realization if and only if $\lambda$ is a quasi-exponential function. The scalar field $\varphi$ is allowed to be any smooth field.

\subsubsection{When is the Short Rate a Markov Process?}

One of the classical problems concerning the HJM approach to interest rate modeling is that of determining when a given forward rate model is realized by a short rate model, i.e. when the short rate is Markovian. We now briefly indicate how the theory developed above can be used in order to analyze this question. For the full theory see [9].

Using the results above, we immediately have the following general necessary condition.

Proposition 4.8 The forward rate model generated by $\sigma$ is a generic short rate model, i.e the short rate is generically a Markov process, only if

$$
\operatorname{dim}\{\mu, \sigma\}_{L A} \leq 2
$$


Proof. If the model is really a short rate model, then bond prices are given as $p_{t}(x)=F\left(t, R_{t}, x\right)$ where $F$ solves the term structure PDE. Thus bond prices, and forward rates are generated by a two dimensional factor model with time $t$ and the short rate $R$ as the state variables.

Remark 4.3 The most natural case is $\operatorname{dim}\{\mu, \sigma\}_{L A}=2$. It is an open problem whether there exists a non-deterministic generic short rate model with $\operatorname{dim}\{\mu, \sigma\}_{L A}=1$.

Note that condition (119) is only a sufficient condition for the existence of a short rate realization. It guarantees that there exists a two-dimensional realization, but the question remains whether the realization can chosen in such a way that the short rate and running time are the state variables. This question is completely resolved by the following central result.

Theorem 4.3 Assume that the model is not deterministic, and take as given a time invariant volatility $\sigma(r, x)$. Then there exists a short rate realization if and only if the vector fields $[\mu, \sigma]$ and $\sigma$ are parallel, i.e. if and only if there exists a scalar field $\alpha(r)$ such that the following relation holds (locally) for all $r$.

$$
[\mu, \sigma](r)=\alpha(r) \sigma(r) .
$$

Proof. See [9].

It turns out that the class of generic short rate models is very small indeed. We have, in fact, the following result, which was first proved in [31] (using techniques different from those above). See [9] for a proof based on Theorem 4.3.

Theorem 4.4 Consider a HJM model with one driving Wiener process and a volatility structure of the form

$$
\sigma(r, x)=g(R, x) .
$$

where $R=r(0)$ is the short rate. Then the model is a generic short rate model if and only if $g$ has one of the following forms.

- There exists a constant $c$ such that

$$
g(R, x) \equiv c .
$$

- There exist constants a and $c$ such that.

$$
g(R, x)=c e^{-a x} .
$$


- There exist constants $a$ and $b$, and a function $\alpha(x)$, where $\alpha$ satisfies a certain Riccati equation, such that

$$
g(R, x)=\alpha(x) \sqrt{a R+b}
$$

We immediately recognize these cases as the Ho-Lee model, the Hull-White extended Vasiček model, and the Hull-White extended Cox-Ingersoll-Ross model. Thus, in this sense the only generic short rate models are the affine ones, and the moral of this, perhaps somewhat surprising, result is that most short rate models considered in the literature are not generic but "accidental". To understand the geometric picture one can think of the following program.

1. Choose an arbitrary short rate model, say of the form

$$
d R_{t}=a\left(R_{t}\right) d t+b\left(R_{t}\right) d W_{t}
$$

with a fixed initial point $R_{0}$.

2. Solve the associated PDE in order to compute bond prices. This will also produce:

- An initial forward rate curve $\hat{r}^{o}(x)$.

- Forward rate volatilities of the form $g(R, x)$.

3. Forget about the underlying short rate model, and take the forward rate volatility structure $g(R, x)$ as given in the forward rate equation.

4. Initiate the forward rate equation with an arbitrary initial forward rate curve $r^{o}(x)$

The question is now whether the thus constructed forward rate model will produce a Markovian short rate process. Obviously, if you choose the initial forward rate curve $r^{o}$ as $r^{o}=\hat{r}^{o}$, then you are back where you started, and everything is OK. If, however, you choose another initial forward rate curve than $\hat{r}^{o}$, say the observed forward rate curve of today, then it is no longer clear that the short rate will be Markovian. What the theorem above says, is that only the models listed above will produce a Markovian short rate model for all initial points in a neighborhood of $\hat{r}^{o}$. If you take another model (like, say, the Dothan model) then a generic choice of the initial forward rate curve will produce a short rate process which is not Markovian.

\subsection{Notes}

The section is based on [9] where full proofs and further results can be found, and where also the time varying case is considered. In our study of the constant direction model above, $\varphi$ was allowed to be any smooth functional of the entire 
forward rate curve. The simpler special case when $\varphi$ is a point evaluation of the short rate, i.e. of the form $\varphi(r)=h(r(0))$ has been studied in [1], [29] and [35]. All these cases falls within our present framework and, the results are included as special cases of the general theory above. A different case, treated in [14], occurs when $\sigma$ is a finite point evaluation, i.e. when $\sigma(t, r)=h\left(t, r\left(x_{1}\right), \ldots r\left(x_{k}\right)\right)$ for fixed benchmark maturities $x_{1}, \ldots, x_{k}$. In [14] it is studied when the corresponding finite set of benchmark forward rates is Markovian.

A classic paper on Markovian short rates is [13], where a deterministic volatility of the form $\sigma(t, x)$ is considered. Theorem 4.4 was first stated and proved in [31]. See [18] for an example with a driving Levy process.

The geometric ideas presented above and in [9] are intimately connected to controllability problems in systems theory, where they have been used extensively (see [30]). They have also been used in filtering theory, where the problem is to find a finite dimensional realization of the unnormalized conditional density process, the evolution of which is given by the Zakai equation. See [12] for an overview of these areas.

\section{Constructing Realizations}

The purpose of this section is to present a systematic procedure for the construction of finite dimensional realizations for any model possessing a finite dimensional realization.

\subsection{The Construction Algorithm}

The method basically works as follows: From Theorem 4.2 we know that there exists an FDR if and only if the Lie algebra $\{\mu, \sigma\}_{L A}$ is finite dimensional. Given a set of generators for this Lie algebra we now show how to construct an FDR by essentially solving a finite number of ordinary differential equations in Hilbert space. The method will work for any Hilbert space SDE of the form (115) with smooth drift and diffusion vector fields, and in particular it can be applied to the forward rate equation.

Let us assume that the Lie algebra $\{\mu, \sigma\}_{L A}$, is finite dimensional near the point $r^{o}$. Then a finite dimensional realization can be constructed in the following way:

- Choose an involutive system of independent vector fields $f_{1}, \ldots, f_{d}$ which span $\{\mu, \sigma\}_{L A}$. Lemma 4.1 is often useful for simplifying the vector fields.

- Compute the invariant manifold $G\left(z_{1}, \ldots, z_{d}\right)$ using Proposition 4.2.

- Since $\mathcal{G}$ is invariant under $r$, we now know that $r_{t}=G\left(Z_{t}\right)$ for some state process $Z$. We thus make the following Ansatz for the dynamics of the 
state space variables $Z$

$$
d Z_{t}=a\left(Z_{t}\right) d t+b\left(Z_{t}\right) \circ d W_{t} .
$$

- From the Stratonovich version of the Itô formula it then follows that

$$
G_{\star} a=\mu, \quad G_{\star} b=\sigma .
$$

- Use the equations in (121) to solve for the vector fields $a$ and $b$.

Before going on to concrete applications, let us make some remarks.

\section{Remark 5.1}

- We know that there will always exist solutions, a and b, to(121).

- It may be that the equations in (121) do not have unique solutions, but for us it is enough to find one solution, and any solution will do.

- Although we have to solve for the Stratonovich dynamics of the state variables, it turns out that the Itô-dynamics are typically much nicer looking (see below). This is not surprising since this is also true for the forward rate dynamics themselves.

Again we emphasize that this method can be applied quite mechanically, the only choice to be made is that of vector fields which span the Lie algebra $\{\mu, \sigma\}_{L A}$. Generally we will want to choose these vector fields as simple as possible and to do this we use Lemma 4.1. The reason why we want simple vector fields is that this simplifies the computation of the parameterization of the forward rate curves in the next step (recall that this requires solving $\mathcal{H}$-valued ODEs with right hand sides equal to the generating vector fields).

In the next few sections we will apply this scheme repeatedly to various volatilities $\sigma$ and derive finite dimensional realizations.

\subsection{Deterministic volatility}

Assume that

$$
\sigma(r, x)=\sigma(x),
$$

where each component of the vector $\sigma$ is of the following form

$$
\sigma_{i}(x)=\sigma_{i} \lambda_{i}(x), \quad i=1, \ldots, m
$$

Here, with a slight abuse of notation, $\sigma_{i}$ on the right hand side denotes a constant, and $\lambda_{i}$ is a constant vector field. We know from Proposition 4.4 that the 
forward rate equation generated by this volatility structure has a finite dimensional realization if and only if

$$
\operatorname{dim}\left(\operatorname{span}\left\{\sigma, \mathbf{F} \sigma, \mathbf{F}^{2} \sigma, \ldots\right\}\right)<\infty,
$$

where $\mathbf{F}$ denotes the operator $\frac{\partial}{\partial x}$. We therefore assume that $\lambda_{i}$ solves the ODE

$$
\mathbf{F}^{n_{i}+1} \lambda_{i}(x)=\sum_{k=0}^{n_{i}} c_{k}^{i} \mathbf{F}^{k} \lambda_{i}(x)
$$

where the $c_{k}^{i}$ :s are constants. Since the Lie algebra spanned by $\mu$ and $\sigma$ for this case is given by

$$
\{\mu, \sigma\}_{L A}=\operatorname{span}\left\{\mu, \sigma, \mathbf{F} \sigma, \mathbf{F}^{2} \sigma, \ldots\right\},
$$

we can choose the following generator system for the Lie algebra

$$
\{\mu, \sigma\}_{L A}=\operatorname{span}\left\{\mu, \mathbf{F}^{k} \lambda_{i} ; i=1, \ldots, m ; k=0,1, \ldots, n_{i}\right\} .
$$

The next step in constructing a finite dimensional realization is to compute the invariant manifold $G\left(z_{0}, z_{k}^{i} ; i=1, \ldots, m ; k=0,1, \ldots, n_{i}\right)$. This means computing the operators $\exp \{\mu t\}$ and $\exp \left\{\mathbf{F}^{k} \lambda_{i}\right\}, i=1, \ldots, m, k=0, \ldots, n_{i}$. This has been done in Proposition 4.5 and the invariant manifold generated by the initial forward rate curve $r_{0}$ is parameterized as

$$
\begin{aligned}
& G\left(z_{0}, z_{k}^{i} ; i=1, \ldots, m ; k=0,1, \ldots, n_{i}\right)(x) \\
= & r_{0}\left(x+z_{0}\right)+\frac{1}{2}\left(\left\|S\left(x+z_{0}\right)\right\|^{2}-\|S(x)\|^{2}\right)+\sum_{i=1}^{m} \sum_{k=0}^{n_{i}} \mathbf{F}^{k} \lambda_{i}(x) z_{k}^{i},
\end{aligned}
$$

where

$$
S(x)=\int_{0}^{x} \sigma(u) d u .
$$

We now proceed to the last step of the procedure, which is finding the dynamics of the state space variables. This means solving the equations (121). We therefore need the Frechet derivative $G^{\prime}$ of $G$. Simple calculations give

$$
\begin{aligned}
G^{\prime}\left(z_{0}, z_{k}^{i} ; i=1, \ldots, m ; k=0,1, \ldots, n_{i}\right)\left(\begin{array}{c}
h_{0} \\
h_{0}^{1} \\
h_{1}^{1} \\
\vdots \\
h_{n_{m}}^{m}
\end{array}\right)(x) \\
=\frac{\partial}{\partial x} r_{0}\left(x+z_{0}\right) h_{0}+D\left(x+z_{0}\right) h_{0}+\sum_{i=1}^{m} \sum_{k=0}^{n_{i}} \mathbf{F}^{k} \lambda_{i}(x) h_{k}^{i},
\end{aligned}
$$

where $D$ is the constant field given by

$$
D(x)=\sum_{i=1}^{m} \sigma_{i}^{2} \lambda_{i}(x) \int_{0}^{x} \lambda_{i}(u) d u .
$$


Since for this model the Frechet derivative with respect to $r$ of each component of the volatility is zero, i.e. $\sigma_{i}^{\prime}(r, x)=0$, we obtain the following expression for $\mu$.

$$
\mu(r)=\mathbf{F} r+D
$$

If we use that $r=G(z)$ we can obtain an expression for $\mathbf{F} r$, and the equation $G_{\star} a=\mu$ then reads

$$
\begin{aligned}
& \frac{\partial}{\partial x} r_{0}\left(x+z_{0}\right) a_{0}+D\left(x+z_{0}\right) a_{0}+\sum_{j=1}^{m} \sum_{k=0}^{n_{j}} \mathbf{F}^{k} \lambda_{j}(x) a_{j k} \\
= & \frac{\partial}{\partial x} r_{0}\left(x+z_{0}\right)+D\left(x+z_{0}\right)+\sum_{j=1}^{m} \sum_{k=0}^{n_{j}} \mathbf{F}^{k+1} \lambda_{j}(x) z_{k}^{j} .
\end{aligned}
$$

Since this equality is to hold for all $x$, and $a$ is not allowed to depend on $x$ it is possible to identify what $a$ must look like. If we recall that $\lambda_{i}$ solves the ODE defined in (124) we obtain

$$
\begin{array}{rlrl}
a_{0} & =1, & \\
a_{j 0}=c_{0}^{j} z_{n_{j}}^{j}, & j=1, \ldots, m, \\
a_{j k}=c_{k}^{j} z_{n_{j}}^{j}+z_{k-1}^{j}, & j=1, \ldots, m ; k=1, \ldots, n_{j} .
\end{array}
$$

From $G_{\star} b^{i}(z)(x)=\sigma_{i}(x)$ we obtain the equation

$$
\begin{aligned}
& \frac{\partial}{\partial x} r_{0}\left(x+z_{0}\right) b_{0}^{i}+D\left(x+z_{0}\right) b_{0}^{i}+\sum_{j=1}^{m} \sum_{k=0}^{n_{j}} \mathbf{F}^{k} \lambda_{j}(x) b_{j k}^{i} \\
= & \sigma_{i} \lambda_{i}(x),
\end{aligned}
$$

where $\sigma_{i}$ denotes a constant. Therefore we have that

$$
\begin{array}{ll}
b_{j k}^{i}=\sigma_{i}, & j=i, k=0, \\
b_{j k}^{i}=0, & \text { all other } j \text { and } k .
\end{array}
$$

From this we see that to each Wiener process there corresponds one state variable which is driven by this, and only this, Wiener process. The dynamics for these state variables are given by

$$
d Z_{0}^{j}=c_{0}^{j} Z_{n_{j}}^{j} d t+\sigma_{j} \circ d W_{t}^{j}, \quad j=1, \ldots, m .
$$

Since $\sigma_{j}$ is a constant, the Itô-dynamics will look the same, and we have thus proved the following proposition.

Proposition 5.1 Given the initial forward rate curve $r_{0}$ the forward rate system generated by the volatilities described in equations (122) through (124) has a finite dimensional realization given by

$$
r_{t}=G\left(Z_{t}\right),
$$


where $G$ was defined in (125) and the dynamics of the state space variables $Z$ are given by

$$
\left\{\begin{aligned}
d Z_{0} & =d t \\
d Z_{0}^{j} & =c_{0}^{j} Z_{n_{j}}^{j} d t+\sigma_{j} d W_{t}^{j}, \quad j=1, \ldots, m, \\
d Z_{k}^{j} & =\left(c_{k}^{j} Z_{n_{j}}^{j}+Z_{k-1}^{j}\right) d t, \quad j=1, \ldots, m ; k=1, \ldots, n_{j} .
\end{aligned}\right.
$$

Remark 5.2 Note that the first state space variable represents running time. This will be the case for all realizations derived below.

\subsubsection{Ho-Lee}

As a special case of the deterministic volatilities studied in the previous section consider a volatility given by

$$
\sigma(x)=\sigma,
$$

where $\sigma$ is a scalar constant, that is we have only one driving Wiener process. In the formalism of the previous paragraph we have $\lambda(x) \equiv 1$, which satisfies the trivial ODE $\mathbf{F} \lambda(x)=0$. A direct application of Proposition 5.1 gives the following result.

Proposition 5.2 Given the initial forward rate curve $r_{0}$ the forward rate system generated by the volatility of equation (126) has a finite dimensional realization given by

$$
r_{t}=G\left(Z_{t}\right),
$$

where $G$ is given by

$$
G\left(z_{0}, z_{1}\right)(x)=r_{0}\left(x+z_{0}\right)+\sigma^{2}\left(x z_{0}+\frac{1}{2} z_{0}^{2}\right)+z_{1},
$$

and the dynamics of the state space variables $Z$ are given by

$$
\left\{\begin{array}{l}
d Z_{0}(t)=d t, \\
d Z_{1}(t)=\sigma d W_{t} .
\end{array}\right.
$$

\subsubsection{Hull-White}

Another special case of deterministic volatilities is

$$
\sigma(x)=\sigma e^{-c x},
$$

where $\sigma$ and $c$ are scalar constants, so again there is only one driving Wiener process. This time we have $\lambda(x)=e^{-c x}$, which satisfies the ordinary differential equation $\mathbf{F} \lambda(x)=-c \lambda(x)$. Applying Proposition 5.1 once more we obtain the following. 
Proposition 5.3 Given the initial forward rate curve $r_{0}$ the forward rate system generated by the volatility of equation (127) has a finite dimensional realization given by

$$
r_{t}=G\left(Z_{t}\right)
$$

where $G$ is given by

$$
G\left(z_{0}, z_{1}\right)(x)=r_{0}\left(x+z_{0}\right)+\frac{\sigma^{2}}{c^{2}}\left(e^{-c x}\left(1-e^{-c z_{0}}\right)+\frac{e^{-2 c x}}{2}\left(e^{-2 c z_{0}}-1\right)\right)+z_{1},
$$

and the dynamics of the state space variables $Z$ are given by

$$
\left\{\begin{array}{l}
d Z_{0}(t)=d t \\
d Z_{1}(t)=-c Z_{1}(t) d t+\sigma d W_{t} .
\end{array}\right.
$$

\subsection{Deterministic direction volatility}

Consider a volatility structure of the form

$$
\sigma(r, x)=\varphi(r) \lambda(x) .
$$

Here $\varphi$ is a smooth functional of $r$, and $\lambda$ is a constant vector field. Note that we are now dealing with the case with only one driving Wiener process. Depending on whether $\varphi$ satisfies a certain non-degeneracy condition or not we get two cases. We next study these two cases separately.

\subsubsection{The generic case}

In the generic case $\varphi$ satisfies the following assumption.

Assumption 5.1 We assume that

- $\varphi(r) \neq 0$ for all $r \in \mathcal{H}$ and for all $i=1, \ldots, m$.

- $\Phi^{\prime \prime}(r)[\lambda ; \lambda] \neq 0$ for all $r \in \mathcal{H}$, where $\Phi(r)=\varphi^{2}(r)$ and $\Phi^{\prime \prime}(r)[\lambda ; \lambda]$ denotes the second order Frechet derivative of $\Phi$ operating on $[\lambda ; \lambda]$.

Given these assumptions, Proposition 6.1 in [9] states that the system of forward rates generated by the volatility (128) possesses a finite dimensional realization if and only if $\lambda$ is a quasi-exponential function, i.e. of the form $\lambda(x)=c e^{A x} b$, where $c$ is a row vector, $A$ is a square matrix and $b$ is a column vector. We will therefore assume that $\lambda$ is of the form

$$
\lambda(x)=p(x) e^{\alpha x}
$$

where $p$ is a polynomial of degree $n$ and $\alpha$ is a scalar constant. 
It is also shown in [9] that, given Assumption 5.1, the Lie algebra generated by $\mu$ and $\sigma$ is given by

$$
\{\mu, \sigma\}_{L A}=\operatorname{span}\left\{\mathbf{F} r, \mathbf{F}^{i} \lambda, \mathbf{F}^{i} D ; i=0,1, \ldots\right\},
$$

where

$$
D(x)=\lambda(x) \int_{0}^{x} \lambda(u) d u .
$$

We may now note that $\lambda$, regardless of what $p$ looks like, satisfies the following ODE of order $n+1$

$$
(\mathbf{F}-\alpha)^{n+1} \lambda(x)=0 .
$$

This can also be written in the following way

$$
\mathbf{F}^{n+1} \lambda(x)=-\sum_{i=0}^{n}\left(\begin{array}{c}
n+1 \\
i
\end{array}\right)(-\alpha)^{n+1-i} \mathbf{F}^{i} \lambda(x) .
$$

Partial integration reveals that $D$ can be written as $D(x)=u(x) e^{2 \alpha x}+\gamma \lambda(x)$, where $u$ is a polynomial of degree $q=2 n$ and $\gamma$ is a constant. Using Lemma 4.1 we see that we can use $\widetilde{D}$ instead of $D$ to generate the Lie algebra, where $\widetilde{D}$ is given by

$$
\widetilde{D}(x)=D(x)-\left[\sum_{i=0}^{n}\left(\frac{-1}{\alpha}\right)^{i+1} \mathbf{F}^{i} p(0)\right] \cdot \lambda(x) .
$$

Here the sum on the right hand side equals $\gamma$. Therefore $\widetilde{D}(x)=u(x) e^{2 \alpha x}$ and thus $\widetilde{D}$ satisfies the following ODE of order $q+1$

$$
(\mathbf{F}-2 \alpha)^{q+1} \widetilde{D}(x)=0,
$$

which we can also write as

$$
\mathbf{F}^{q+1} \widetilde{D}(x)=-\sum_{j=0}^{q}\left(\begin{array}{c}
q+1 \\
j
\end{array}\right)(-2 \alpha)^{q+1-j} \mathbf{F}^{j} \widetilde{D}(x) .
$$

After these considerations we choose the following generator system for the Lie algebra

$$
\{\mu, \sigma\}_{L A}=\operatorname{span}\left\{\mathbf{F} r, \mathbf{F}^{i} \lambda, \mathbf{F}^{j} \widetilde{D} ; i=0,1, \ldots, n ; j=0,1, \ldots, q\right\},
$$

We now turn to the task of finding a parameterization of the invariant manifold $G\left(z_{0}, z_{i}^{1}, z_{j}^{2} ; i=0,1, \ldots, n ; j=0,1, \ldots, q\right)$, which amounts to computing the operators $\exp \{\mathbf{F} r t\} \exp \left\{\mathbf{F}^{i} \lambda t\right\}, i=0,1, \ldots, n$ and $\exp \left\{\mathbf{F}^{j} \widetilde{D} t\right\}, j=0,1, \ldots, q$. The operator $\exp \{\mathbf{F} r t\}$ is obtained as the solution to

$$
\frac{d y_{t}}{d t}=\mathbf{F} r
$$


This is a linear equation and the solution is

$$
y_{t}=e^{\mathbf{F} t} y_{0},
$$

which means that

$$
\left(e^{\mathbf{F} t} r_{0}\right)(x)=r_{0}(x+t) .
$$

Since the rest of the generating fields are constant, the corresponding ODEs are trivial, and we have

$$
\left(e^{\mathbf{F}^{i} \lambda t} r_{0}\right)(x)=r_{0}(x)+\mathbf{F}^{i} \lambda t,
$$

and

$$
\left(e^{\mathbf{F}^{j} \widetilde{D} t} r_{0}\right)(x)=r_{0}(x)+\mathbf{F}^{j} \tilde{D} t,
$$

respectively. The invariant manifold generated by the initial forward rate curve $r_{0}$ is thus parameterized as

$$
\begin{aligned}
& G\left(z_{0}, z_{i}^{1}, z_{j}^{2} ; i=0,1, \ldots, n ; j=0,1, \ldots, q\right)(x) \\
= & r\left(x+z_{0}\right)+\sum_{i=0}^{n} \mathbf{F}^{i} \lambda(x) z_{i}^{1}+\sum_{j=0}^{q} \mathbf{F}^{j} \widetilde{D}(x) z_{j}^{2} .
\end{aligned}
$$

To obtain the state space dynamics we solve the equations (121). The Frechet derivative $G^{\prime}$ of $G$ is given by

$$
\begin{gathered}
G^{\prime}\left(z_{0}, z_{i}^{1}, z_{j}^{2} ; i=0,1, \ldots, n ; j=0,1, \ldots, q\right)\left(\begin{array}{c}
h_{0} \\
h_{0}^{1} \\
h_{1}^{1} \\
\vdots \\
h_{q}^{2}
\end{array}\right)(x) \\
=\frac{\partial}{\partial x} r_{0}\left(x+z_{0}\right) h_{0}+\sum_{i=0}^{n} \mathbf{F}^{i} \lambda(x) h_{i}^{1}+\sum_{j=0}^{q} \mathbf{F}^{j} \widetilde{D}(x) h_{j}^{2} .
\end{gathered}
$$

We have the following expression for $\mu$

$$
\mu(r)=\mathbf{F} r+\varphi^{2}(r) D-\frac{1}{2} \varphi^{\prime}(r)[\lambda] \varphi(r) \lambda,
$$

where $D$ was defined in (130). Using that $r=G(z)$, the equation $G_{\star} a=\mu$ reads

$$
\begin{aligned}
& \frac{\partial}{\partial x} r_{0}\left(x+z_{0}\right) a_{0}+\sum_{i=0}^{n} \mathbf{F}^{i} \lambda(x) a_{i}^{1}+\sum_{j=0}^{q} \mathbf{F}^{j} \widetilde{D}(x) a_{j}^{2} \\
= & \frac{\partial}{\partial x} r_{0}\left(x+z_{0}\right)+\sum_{i=0}^{n} \mathbf{F}^{i+1} \lambda(x) z_{i}^{1}+\sum_{j=0}^{q} \mathbf{F}^{j+1} \widetilde{D}(x) z_{j}^{2} \\
& +\varphi^{2}(G(z)) D(x)-\frac{1}{2} \varphi^{\prime}(G(z))[\lambda] \varphi(G(z)) \lambda(x) .
\end{aligned}
$$


This equality has to hold for all $x$, and $a$ is not allowed to depend on $x$. This allows us to identify what $a$ must look like. Recall that $\lambda$ solves the ODE defined in (131), and that $\widetilde{D}$ solves the ODE in (132). Furthermore, recall that $D(x)=$ $\widetilde{D}(x)+\gamma \lambda(x)$, and let

$$
c_{i}=-\left(\begin{array}{c}
n+1 \\
i
\end{array}\right)(-\alpha)^{n+1-i} \text { and } \quad d_{j}=-\left(\begin{array}{c}
q+1 \\
j
\end{array}\right)(-2 \alpha)^{q+1-j} .
$$

We then obtain

$$
\begin{array}{lll}
a_{0} & =1 & \\
a_{0}^{1}=c_{0} z_{n}^{1}+\gamma \varphi^{2}(G(z))-\frac{1}{2} \varphi^{\prime}(G(z))[\lambda] \varphi(G(z)), & \\
a_{i}^{1}=c_{i} z_{n}^{1}+z_{i-1}^{1}, & i=1, \ldots, n, \\
a_{0}^{2}=d_{0} z_{q}^{2}+\varphi^{2}(G(z)), & j=1, \ldots, q . \\
a_{j}^{2}=d_{j} z_{q}^{2}+z_{j-1}^{2}, &
\end{array}
$$

From $G_{\star} b=\sigma$ we obtain the equation

$$
\begin{aligned}
& \frac{\partial}{\partial x} r_{0}\left(x+z_{0}\right) b_{0}+\sum_{i=0}^{n} \mathbf{F}^{i} \lambda(x) b_{i}^{1}+\sum_{j=0}^{q} \mathbf{F}^{j} \widetilde{D}(x) b_{j}^{2} \\
= & \varphi(G(z)) \lambda(x),
\end{aligned}
$$

where we have used that $r=G(z)$. This gives us

$$
\begin{array}{rlrl}
b_{0} & =0, & \\
b_{j}^{i} & =\varphi(G(z)), & & i=1, j=0, \\
b_{j}^{i} & =0, & & \text { all other } i, j .
\end{array}
$$

Just as for the case with deterministic volatilities we see that the Wiener process only drives one of the state variables. On Stratonovich form the dynamics of $Z_{0}^{1}$ are

$$
d Z_{0}^{1}=\left(c_{0} Z_{n}^{1}+\gamma \varphi^{2}(G(Z))-\frac{1}{2} \varphi^{\prime}(G(Z))[\lambda] \varphi(G(Z))\right) d t+\varphi(G(Z)) \circ d W_{t} .
$$

Changing to Itô-dynamics for $Z_{0}^{1}$ we have the following proposition.

Proposition 5.4 Given the initial forward rate curve $r_{0}$ the forward rate system generated by the volatility defined by the equations (128) and (129) has a finite dimensional realization given by

$$
r_{t}=G\left(Z_{t}\right),
$$


where $G$ was defined in (133) and the dynamics of the state space variables $Z$ are given by

$$
\left\{\begin{aligned}
d Z_{0} & =d t \\
d Z_{0}^{1} & =\left[c_{0} Z_{n}^{1}+\gamma \varphi^{2}(G(Z))\right] d t+\varphi(G(Z)) d W_{t}, \\
d Z_{i}^{1} & =\left(c_{i} Z_{n}^{1}+Z_{i-1}^{1}\right) d t, \quad i=1, \ldots, n \\
d Z_{0}^{2} & =\left[d_{0} Z_{q}^{2}+\varphi^{2}(G(Z))\right] d t \\
d Z_{j}^{2} & =\left(d_{j} Z_{q}^{2}+Z_{j-1}^{2}\right) d t, \quad j=1, \ldots, q .
\end{aligned}\right.
$$

Here $c_{i}$ and $d_{j}$ are given by (134)

\section{The Filipovic and Teichmann Extension}

While in one sense the general FDR problem is more or less completely solved using the Lie algebra methodology of [9] described above, we still have a major technical problem to tackle. This has to do with the fact that in the approach above, the framework was that of strong solutions of infinite dimensional SDEs in Hilbert space and this forced us to construct the particular Hilbert space $\mathcal{H}$ of real analytic functions as the space of forward rate curves. While serving reasonably well, it was even at an early stage clear that this particular space was very small, and in particular it was pointed out by Filipović and Teichmann that the space does not include the forward rate curves generated by the CoxIngersoll-Ross model (see [15]). It was therefore necessary to extend the theory to a larger space but such an extension is far from to trivial to carry out, the problem being that on a larger Hilbert space you will loose the smoothness of the differential operator $\partial / \partial x$ appearing in the drift term of the forward rate equation. This problem was overcome with great elegance by Filipović and Teichmann who, partly building on the geometric and analytic results from [22], in [23] managed to extend the Lie algebraic FDR theory to a much larger space of forward rate curves than the space $\mathcal{H}$ considered in [9]. In doing so, Filipović and Teichmann first extend the space of [9] to a much larger Hilbert space. On the new space, however, the operator $\partial / \partial x$ becomes unbounded so they then change the topology on the space, thus making it into a Frechet space where the operator in fact is bounded. This approach, however, leads to new problems, since on a Frechet space there is no easy way of introducing differential calculusin fact there is even no obvious way of defining the concept of smoothness which is necessary in order to have a Frobenius theorem. In order to overcome this problem, Filipović and Teichmann used the framework of so called "convenient spaces" developed some ten years ago (see [23] for references) in order to carry out analysis on the enlarged space. The main result of all this is that the Lie algebra conditions obtained by Björk and Svensson are shown to still hold in this more general setting. At this point it is worth mentioning that the technical 
price one has to pay for going into the deep parts of the theory of convenient analysis is quite high. It is therefore fortunate that the Lie algebraic machinery of [23] can be used without going into these (sometimes very hard) technical details. In fact, one of the main result of [23] can be formulated in the following pedestrian terms for the working mathematician: "When you are searching for FDRs for equations of HJM type, you can compute the relevant Lie algebra without worrying about the space, since Filipovic and Teichmann will always provide you with a convenient space to work in". In [23] and in the follow up papers [24] and [25] the extended Lie algebra theory in [23] is used in to analyze a number of concrete problems concerning the forward rate equation: In particular, Filipović and Teichmann prove the remarkable result that any forward rate model admitting an FDR must necessarily have an affine term structure.

\section{Stochastic Volatility Models}

We now extend the theory developed above to include stochastic volatility models. More precisely we will study HJM models of the forward rates in which the volatility, apart from being dependent on the present forward rate curve, also is allowed to be modulated by a $k$ dimensional hidden Markov process $y$. The model is defined as follows.

Definition 7.1 The Itô formulation of the stochastic volatility model (henceforth SVM) is defined as the process pair $(r, y)$, where the $Q$-dynamics of $r$ and $y$ are defined by the following system of SDEs.

$$
\begin{aligned}
d r_{t}(x) & =\left\{\frac{\partial}{\partial x} r_{t}(x)+\mathbf{H} \sigma\left(r_{t}, y_{t}, x\right)\right\} d t+\sigma\left(r_{t}, y_{t}, x\right) d W_{t} \\
d y_{t} & =a^{0}\left(y_{t}\right) d t+b\left(y_{t}\right) d W_{t}
\end{aligned}
$$

where $\mathbf{H}$ is defined by

$$
\mathbf{H} \sigma(r, y, x)=\sigma(r, y, x) \int_{0}^{x} \sigma^{\star}(r, y, s) d s,
$$

and ${ }^{\star}$ denotes transpose.

In this specification we consider the following objects as given a priori:

- The volatility structure $\sigma$ for the forward rates, i.e. a deterministic mapping

$$
\sigma: \mathcal{H} \times R^{k} \times R_{+} \rightarrow R^{m}
$$

- The drift vector field $a^{0}$ for $y$, i.e. a deterministic mapping

$$
a^{0}: R^{k} \rightarrow R^{k} \text {. }
$$


(The superscript on $a^{0}$ will be explained below)

- The volatility vector field $b$ for $y$, i.e. a deterministic mapping

$$
b: R^{k} \rightarrow M(k, m)
$$

where $M(k, m)$ denotes the set of $k \times m$ matrices.

We view $\sigma$ as a row vector

$$
\sigma(r, y, x)=\left[\sigma_{1}(r, y, x), \ldots, \sigma_{m}(r, y, x)\right]
$$

the drift $a^{0}$ is viewed as a column vector and the volatility $b$ as a matrix:

$$
a^{0}(y)=\left[\begin{array}{c}
a_{1}^{0}(y) \\
\vdots \\
a_{k}^{0}(y)
\end{array}\right], \quad b(y)=\left[\begin{array}{cccc}
b_{11}(y) & b_{12}(y) & \cdots & b_{1 m}(y) \\
b_{21}(y) & b_{22}(y) & \cdots & b_{2 m}(y) \\
\vdots & \vdots & & \vdots \\
b_{k 1}(y) & b_{k 2}(y) & \cdots & b_{k m}(y)
\end{array}\right]
$$

We note in particular that the forward rate volatility $\sigma$ is allowed to be an arbitrary functional of the entire forward rate curve $r$, as well as a function of the $k$-dimensional variable $y$. We may also view each component of $\sigma$ as a mapping from $\mathcal{H} \times R^{k}$ to a space of functions (parameterized by $x$ ), and we will in fact assume that each $\sigma_{i}$, viewed in this way, is a smooth mapping with values in $\mathcal{H}$, i.e.

$$
\sigma_{i}: \mathcal{H} \times R^{k} \rightarrow \mathcal{H}
$$

We make the following regularity assumptions.

Assumption 7.1 From now on we assume that:

- The mappings $\sigma_{i}: \mathcal{H} \times R^{k} \rightarrow \mathcal{H}$ are smooth for $i=1, \ldots, m$.

- The mapping $\mathbf{H} \sigma: \mathcal{H} \times R^{k} \rightarrow \mathcal{H}$, defined by (137) is smooth.

- The mappings $a^{0}$ and $b$ are smooth on $R^{k}$.

In the forward rate dynamics (135) we recognize the drift term in the $r$-dynamics above as the HJM drift condition, transferred into the Musiela parameterization. Note the particular structure of the equations (135)-(136): The $y$-process is feeding the drift and diffusion terms of the $r$-dynamics, but the $r$-process does not appear in the $y$-dynamics. Thus the $y$ process is a Markov process in its own right, but this is not the case for the $r$-process. The extended process $\hat{r}=(r, y)$ is, however, Markovian.

In many applications it is natural to study, not only the full SVM above but also a restricted model, where we forget about the dynamics of $y$ and consider $y$ as a constant parameter. In this way we obtain a parameterized model, and the formal definition is as follows. 
Definition 7.2 Consider the SVM defined by (135)-(136) above. For any fixed value of $y \in R^{k}$, the induced parameterized forward rate model is defined by the dynamics

$$
d r_{t}^{y}(x)=\left\{\frac{\partial}{\partial x} r_{t}^{y}(x)+\mathbf{H} \sigma\left(r_{t}^{y}, y, x\right)\right\} d t+\sigma\left(r_{t}^{y}, y, x\right) d W_{t} .
$$

Note that in the parameterized model, the forward rate process $r^{y}$ itself is Markovian, whereas this is not the case in the full stochastic volatility model. For ease of reading we will sometimes drop the superscript ${ }^{y}$.

\subsection{Problem formulation}

The basic problem to be discussed is under what conditions the, inherently infinite dimensional, SVM defined above by (135)-(136), with given initial conditions $r_{0}=r^{0}, y_{0}=y^{0}$, admits a generic finite dimensional Markovian realization in the sense of Section 4. More precisely we thus want to investigate under what conditions the extended process $\hat{r}_{t}=\left(r_{t}, y_{t}\right)$ possesses a local representation of the form

$$
\hat{r}_{t}=\hat{G}\left(Z_{t}\right), \quad Q-\text { a.s. }
$$

where, for some $d, Z$ satisfies a $d$-dimensional SDE of the form

$$
\left\{\begin{aligned}
d Z_{t} & =A_{0}\left(Z_{t}\right) d t+B\left(Z_{t}\right) d W_{t} \\
Z_{0} & =z_{0}
\end{aligned}\right.
$$

and where $\hat{G}$ is a smooth map $G: R^{d} \rightarrow \mathcal{H} \times R^{k}$. The drift and diffusion terms $A_{0}$ and $B$ are assumed to be smooth and of suitable dimensions.

In a realization of this kind, the objects $\hat{G}, A_{0}, B$ and $z_{0}$ will typically depend upon the choice of starting point $\left(r^{0}, y^{0}\right)$. We recall that the term "generic" above means that we demand that there exists a realization, not only for the given initial point $\left(r^{0}, y^{0}\right)$, but in fact for all initial points $\left(r_{0}, y_{0}\right)$ in a neighborhood of $\left(r^{0}, y^{0}\right)$. When we speak of realizations in the sequel we always intend this to mean generic realizations.

Note that the state process $Z$ above is driven by the same Wiener process as the $\hat{r}$ system, and that the realization above is assumed to hold almost surely and trajectory wise.

We may now formulate some natural problems: Main problems:

- Find necessary and sufficient conditions for the existence of an FDR for a given stochastic volatility model.

- Assuming the existence of an FDR has been guaranteed, how do you construct it? 
- How is the existence of an FDR for the full stochastic volatility model related to the existence of an FDR for the induced parameterized model? More precisely: is the existence of an FDR for the parameterized model necessary and/or sufficient for the existence of an FDR for the full model?

\subsection{Test examples: I.}

To illustrate technique, we now present four simple recurrent test examples. In all cases we assume a scalar driving Wiener process $W^{r}$ for the forward rates, a scalar $y$ process and a scalar driving Wiener process $W^{y}$ for the $y$ process. Furthermore we assume that $W^{r}$ and $W^{y}$ are independent. To motivate our choice of examples we recall (see [2]) the following well known (non stochastic) HJM volatilities for the forward rates.

\section{Hull-White extended Vasiček:}

$$
\sigma(r, x)=\sigma e^{-a x} .
$$

Here $a$ and the right hand side occurrence of $\sigma$ are real constants. This HJM model has a short rate realization of the form.

$$
d R_{t}=\left\{\Phi(t)-a R_{t}\right\} d t+\sigma d W_{t},
$$

where the deterministic function $\Phi$ depends on the initial term structure (see [2]). The parameters $\sigma$ and $a$ are the same as in (141).

\section{Hull-White extended Cox-Ingersoll-Ross:}

$$
\sigma(r, x)=\sigma \sqrt{r(0)} \cdot \lambda(x, \sigma, a),
$$

Here $a$ and the right hand side occurrence of $\sigma$ are real constants, whereas $\lambda$ is given by

$$
\lambda(x, \sigma, a)=-\frac{\partial}{\partial x}\left(\frac{2\left(e^{\gamma x}-1\right)}{(\gamma+a)\left(e^{\gamma x}-1\right)+2 \gamma}\right),
$$

where

$$
\gamma=\sqrt{a^{2}+2 \sigma^{2}} .
$$

Also this HJM model admits a short rate realization, namely

$$
d R_{t}=\left\{\Phi(t)-a R_{t}\right\} d t+\sigma \sqrt{R_{t}} d W_{t}
$$

The role of $\Phi$ is as in the extended Vasiček model above.

It is now natural to ask if we can extend these models by allowing one or several parameters to be stochastic, and still retain the existence of a finite dimensional realization. 
We consider the following extensions of the above volatility structures. In all cases we assume that the scalar $y$ process has dynamics of the form

$$
d y_{t}=a_{0}\left(y_{t}\right) d t+b\left(y_{t}\right) d W_{t}^{y},
$$

with $b(y) \neq 0$ for all $y$.

1. HW with stochastic $a$ :

$$
\sigma(r, y, x)=\sigma e^{-y x}
$$

2. HW with stochastic $\sigma$ :

$$
\sigma(r, y, x)=y e^{-a x}
$$

3. CIR with stochastic $\sigma$ :

$$
\sigma(r, y, x)=y \sqrt{r(0)} \cdot \lambda(x, y, a)
$$

4. CIR with stochastic $a$ :

$$
\sigma(r, y, x)=\sigma \sqrt{r(0)} \cdot \lambda(x, \sigma, y)
$$

For all these models, the induced parameterized model admits, by construction, an FDR. It is now reasonable to ask if this also holds for the corresponding stochastic volatility models.

\subsection{Finite realizations for general stochastic volatility mod- els}

In order to solve the FDR problem for stochastic volatility models we will of course use the Lie algebra theory for the existence of FDRs in Hilbert space, developed in Section 4.

\subsubsection{Lie algebra conditions for the existence of an FDR}

Our problem is to study the existence of an FDR for a stochastic volatility model of the form

$$
\begin{aligned}
d r_{t} & =\mu_{0}\left(r_{t}, y_{t}\right) d t+\sigma\left(r_{t}, y_{t}\right) d W_{t} \\
d y_{t} & =a^{0}\left(y_{t}\right) d t+b\left(y_{t}\right) d W_{t} .
\end{aligned}
$$

In the particular case of a forward rate model, the drift term is given by

$$
\mu_{0}(r, y, x)=\frac{\partial}{\partial x} r(x)+\mathbf{H} \sigma(r, y, x)
$$


but none of the results in this section does in fact depend upon this particular structure of $\mu_{0}$. Therefore we will, for the rest of the section, consider a general abstract stochastic volatility model of the form (150)-(151).

To apply our earlier Lie algebra results to the present situation we proceed in the following way.

- Define the Hilbert space $\hat{\mathcal{H}}$ by $\hat{\mathcal{H}}=\mathcal{H} \times R^{k}$.

- Define the $\hat{\mathcal{H}}$-valued process $\hat{r}$ by

$$
\hat{r}_{t}=\left[\begin{array}{l}
r_{t} \\
y_{t}
\end{array}\right]
$$

- Write the dynamics of $\hat{r}$ on Stratonovich form instead of the original Itô form.

- Use the abstract Lie algebraic result from Theorem 4.2 on the process $\hat{r}$.

We will thus view $\hat{r}$ as an infinite dimensional "column vector" process, and we will henceforth always write it on block vector form as above.

The Stratonovich dynamics of $\hat{r}$ are routinely derived as

$$
\begin{aligned}
d r_{t} & =\mu\left(r_{t}, y_{t}\right) d t+\sigma\left(r_{t}, y_{t}\right) \circ d W_{t} \\
d y_{t} & =a\left(y_{t}\right) d t+b\left(y_{t}\right) \circ d W_{t},
\end{aligned}
$$

where

$$
\begin{aligned}
\mu(r, y) & =\mu_{0}(r, y)-\frac{1}{2} \sigma_{r}(r, y) \sigma(r, y)-\frac{1}{2} \sigma_{y}(r, y) b(y) \\
a(y) & =a^{0}(y)-\frac{1}{2} b_{y}(y) b(y) .
\end{aligned}
$$

Here $\sigma_{r}$ denotes the partial Frechet derivative of $\sigma$ w.r.t. the vector variable $r$ and similarly for the other terms.

Written as a single equation on $\hat{\mathcal{H}}$ we thus have

$$
d \hat{r}_{t}=\hat{\mu}(\hat{r}) d t+\hat{\sigma}(\hat{r}) \circ d W_{t},
$$

where $\hat{\mu}$ and $\hat{\sigma}$ are given by

$$
\begin{aligned}
\hat{\mu}(r, y) & =\left[\begin{array}{c}
\mu(r, y) \\
a(y)
\end{array}\right] \\
\hat{\sigma}(r, y) & =\left[\begin{array}{lll}
\hat{\sigma}_{1}(r, y), & \ldots, & \hat{\sigma}_{m}(r, y)
\end{array}\right]
\end{aligned}
$$

Here the vector fields $\hat{\sigma}_{1}, \ldots, \hat{\sigma}_{m}$ are defined by

$$
\hat{\sigma}_{i}(r, y)=\left[\begin{array}{c}
\sigma_{i}(r, y) \\
b_{i}(y)
\end{array}\right]
$$


where $b_{i}$ is the $i:$ th column of the $b$ matrix.

We make the following standing regularity assumption which is assumed to hold throughout the entire paper.

Assumption 7.2 We assume that the dimension (evaluated pointwise) of the Lie algebra

$$
\left\{\hat{\mu}, \hat{\sigma}_{1}, \ldots, \hat{\sigma}_{m}\right\}_{L A}<\infty
$$

is constant in a neighborhood of $\hat{r}_{0} \in \hat{\mathcal{H}}$

Our first general result now follows immediately from Theorem 4.2. Note that when we below speak about the dimension of a Lie algebra, this is always to be interpreted in terms of pointwise evaluation.

Theorem 7.1 Under Assumption 7.2, the stochastic volatility model (150)(151) will have a generic FDR at the point $\hat{r}_{0}$ if and only if

$$
\operatorname{dim}\left\{\hat{\mu}, \hat{\sigma}_{1}, \ldots, \hat{\sigma}_{m}\right\}_{L A}<\infty,
$$

in a neighborhood of $\hat{r}_{0} \in \hat{\mathcal{H}}$.

For simplicity of notation we will often use the shorthand notation $\{\hat{\mu}, \hat{\sigma}\}_{L A}$ for the Lie algebra $\left\{\hat{\mu}, \hat{\sigma}_{1}, \ldots, \hat{\sigma}_{m}\right\}_{L A}$.

\subsubsection{Geometric intuition}

At this level of generality it is hard to obtain more concrete results. As an example: there seems to be no simple result connecting the existence of an FDR for the full model with existence of an FDR for the parameterized model. The geometric intuition behind this is roughly as follows.

- From Proposition 4.1 we know that existence of an FDR for $\hat{r}$ is equivalent to the existence of a finite dimensional invariant manifold in $\hat{\mathcal{H}}$ passing through $\hat{r}_{0}$.

- If the parameterized model admits a generic FDR then, for every fixed $y$ near $y^{0}$, there exists an invariant manifold $\mathcal{G}$ in $\mathcal{H}$ through $r_{0}$. Thus one would perhaps guess that the manifold $\mathcal{G} \times R^{k}$ would be invariant for $\hat{r}$, thus implying the existence of an FDR for $\hat{r}$.

- However, the manifold $\mathcal{G}$ above will generically depend on $y$. Writing it as $\mathcal{G}^{y}$, what may (and generically will) happen is that, as $\hat{r}_{t}$ moves around in $\hat{\mathcal{H}}, y_{t}$ will move in $R^{k}$ and the family $\left\{\mathcal{G}^{y_{t}} ; t \geq 0\right\}$ may sweep out an infinite dimensional manifold in $\hat{\mathcal{H}}$. Thus the existence of an FDR for the parameterized model is not sufficient for the existence of an FDR for the full model. 
- Conversely, the existence of an FDR for the parameterized model does not even seem to be necessary for the existence of an FDR for the full model. Suppose for example that, for each $y$, there does not exist an invariant manifold for the parameterized model. This means that the parameterized model does not possess an FDR. Despite this it could well happen that the process $\hat{r}$ does live on a finite dimensional invariant manifold (and thus possesses an FDR). The reason for this is that there could be a subtle interplay between the dynamics of $r$ and $y$, and in particular one might intuitively expect this interplay to be possible if there is strong correlation between the Wiener process components driving $r$ and $y$.

- From the argument above we are led to guess that the simplest structural situation occurs when $r$ and $y$ are driven by independent Wiener processes. Since in this case, the evolution of $y$ is independent of the present state of $r$, we may even guess (bravely) that any FDR properties of the full model will be "uniform" w.r.t. $y$ in the sense that the results will not depend much on the particular dynamics of $y$.

As we shall see below, the intuition outlined above is basically substantiated.

\subsection{General orthogonal noise models}

Based on the informal arguments in the previous section we now go on to study the case when $r$ and $y$ are driven by independent Wiener processes. We will refer to this type of model as an "orthogonal noise model". We consider the case of a general SDE in Hilbert space.

\subsubsection{Model specification and preliminary results}

Assumption 7.3 For the rest of the section we assume that we can write the Wiener process $W$ on block vector form as

$$
W_{t}=\left[\begin{array}{c}
W_{t}^{r} \\
W_{t}^{y}
\end{array}\right]
$$

where $W^{r}$ and $W^{y}$ are vector Wiener processes of dimensions $m_{r}$ and $m_{y} r e-$ spectively. Furthermore we assume that the $(r, y)$ dynamics are of the particular form

$$
\begin{aligned}
d r_{t} & =\mu_{0}\left(r_{t}, y_{t}\right) d t+\sigma\left(r_{t}, y_{t}\right) d W_{t}^{r} \\
d y_{t} & =a^{0}\left(y_{t}\right) d t+b\left(y_{t}\right) d W_{t}^{y},
\end{aligned}
$$

where the coefficients satisfy suitable smoothness conditions (see Section 7.3). 
Under this assumption $r$ and $y$ are driven by orthogonal noise terms, and this leads to an important simplification of the geometric structure of the model.

Lemma 7.1 The Stratonovich formulation of (164)-(165) is given by

$$
\begin{aligned}
d r_{t} & =\mu\left(r_{t}, y_{t}\right) d t+\sigma\left(r_{t}, y_{t}\right) \circ d W_{t}^{r} \\
d y_{t} & =a\left(y_{t}\right) d t+b\left(y_{t}\right) \circ d W_{t}^{y}
\end{aligned}
$$

where

$$
\begin{aligned}
\mu(r, y) & =\mu_{0}(r, y)-\frac{1}{2} \sigma_{r}(r, y) \sigma(r, y) \\
a(y) & =a^{0}(y)-\frac{1}{2} b_{y}(y) b(y) .
\end{aligned}
$$

Proof. In order to find the Stratonovich form of the $r$ dynamics we need to compute

$$
d\left\langle\sigma, W^{r}\right\rangle_{t}=d \sigma\left(r_{t}, y_{t}\right) .
$$

The infinite dimensional Itô formula gives us

$$
d \sigma\left(r_{t}, y_{t}\right)=(d t \text {-terms })+\sigma_{r}\left(r_{t}, y_{t}\right) \sigma\left(r_{t}, y_{t}\right) d W_{t}^{r}+\sigma_{y}\left(r_{t}, y_{t}\right) b\left(y_{t}\right) d W_{t}^{y}
$$

We thus obtain

$$
d\left\langle\sigma, W^{r}\right\rangle_{t}=\sigma_{r}\left(r_{t}, y_{t}\right) \sigma\left(r_{t}, y_{t}\right) d\left\langle W^{r}, W^{r}\right\rangle_{t}+\sigma_{y}\left(r_{t}, y_{t}\right) b\left(y_{t}\right) d\left\langle W^{y}, W^{r}\right\rangle_{t}
$$

Since $W^{r}$ and $W^{y}$ are independent this simplifies to

$$
d\left\langle\sigma, W^{r}\right\rangle_{t}=\sigma_{r}(r, y) \sigma(r, y) d t .
$$

In order to see more clearly the geometric structure of the orthogonal noise model we write it on block operator form as

$$
d\left[\begin{array}{l}
r_{t} \\
y_{t}
\end{array}\right]=\left[\begin{array}{c}
\mu\left(r_{t}, y_{t}\right) \\
a\left(y_{t}\right)
\end{array}\right] d t+\left[\begin{array}{c}
\sigma\left(r_{t}, y_{t}\right) \\
0
\end{array}\right] \circ d W_{t}^{r}+\left[\begin{array}{c}
0 \\
b\left(y_{t}\right)
\end{array}\right] \circ d W_{t}^{y}
$$

We thus have the following immediate and preliminary result.

Proposition 7.1 The orthogonal noise model (164)-(165) admits an FDR if and only if the Lie algebra generated by the vector fields

$$
\left[\begin{array}{c}
\mu(r, y) \\
a(y)
\end{array}\right],\left[\begin{array}{c}
\sigma_{1}(r, y) \\
0
\end{array}\right], \ldots,\left[\begin{array}{c}
\sigma_{m_{r}}(r, y) \\
0
\end{array}\right],\left[\begin{array}{c}
0 \\
b_{1}(y)
\end{array}\right], \ldots,\left[\begin{array}{c}
0 \\
b_{m_{y}}(y)
\end{array}\right]
$$

is finite dimensional at $\hat{r}_{0}$. 
More compactly we will often write the generators of the Lie algebra above as $\hat{\mu}, \hat{\sigma}$, and $\hat{b}$ where,

$$
\hat{\mu}(r, y)=\left[\begin{array}{c}
\mu(r, y) \\
a(y)
\end{array}\right], \quad \hat{\sigma}(r, y)=\left[\begin{array}{c}
\sigma(r, y) \\
0
\end{array}\right], \quad \hat{b}(y)=\left[\begin{array}{c}
0 \\
b(y)
\end{array}\right]
$$

A very useful property of the orthogonal noise model is the simple structure of the Stratonovich formulation of the parameterized model. The proof is trivial.

Lemma 7.2 For the orthogonal noise model (164)-(165), the Itô formulation of the parameterized model is defined by

$$
d r_{t}=\mu_{0}\left(r_{t}, y\right) d t+\sigma\left(r_{t}, y\right) d W_{t}^{r},
$$

and the Stratonovich formulation of the parameterized model is given by

$$
d r_{t}=\mu\left(r_{t}, y\right) d t+\sigma\left(r_{t}, y\right) \circ d W_{t}^{r}
$$

with $\mu$ defined by (168).

The point of this Lemma is that it shows that, for orthogonal noise models, the operations "restrict to the parameterized model" and "compute the Stratonovich dynamics" commute, i.e. the Stratonovich formulation of the parameterized model is identical to the parameterized version of the Stratonovich formulation of the original model.

In order to obtain easily verifiable necessary and sufficient conditions for the existence of an FDR we will in the next sections introduce some further structural assumptions. In doing this we will have to deal with Lie brackets in several spaces, so we have to clarify some notation.

Definition 7.3 From now on, the following notation is in force:

- For any vector smooth fields $\hat{f}(r, y)$ and $\hat{g}(r, y)$ on $\hat{\mathcal{H}}$, the expression $[\hat{f}, \hat{g}]$ denotes the Lie bracket in $\hat{\mathcal{H}}$.

- For any smooth mapping $f(r, y)$ where $f: \hat{\mathcal{H}} \rightarrow \mathcal{H}$ and for any fixed $y \in R$, the parameterized vector field $f^{y}: \mathcal{H} \rightarrow \mathcal{H}$ is defined by $f^{y}(r)=f(r, y)$

- For any smooth mappings $f, g: \hat{\mathcal{H}} \rightarrow \mathcal{H}$, the expression $\left[f^{y}, g^{y}\right]$ denotes the Lie bracket on $\mathcal{H}$ between $f^{y}$ and $g^{y}$. This Lie bracket will sometimes also be denoted by $[f(\cdot, y), g(\cdot, y)]_{\mathcal{H}}$.

- For vector fields $c(y)$ and $d(y)$ on $R^{k}$, the notation $[c, d]$ denotes the Lie bracket on $R^{k}$. 


\subsubsection{Necessary conditions}

It turns out that, in order to obtain easy necessary condition, a crucial role is played by the geometric relation between the drift vector field $a(y)$ and the Lie algebra on $R^{k}$ generated by the diffusion vector fields $b_{1}(y), \ldots, b_{m_{y}}$.

Our first result relates the stochastic volatility model to the corresponding parameterized model.

Proposition 7.2 Consider the model (164)-(165). Assume that

$$
a \in\left\{b_{1}, \ldots, b_{m_{y}}\right\}_{L A}
$$

in a neighborhood of $y^{0}$. Under this assumption, a necessary condition for the existence of an FDR for the stochastic volatility model is that the corresponding parameterized model

$$
d r_{t}=\mu\left(r_{t}, y\right) d t+\sigma\left(r_{t}, y\right) d W_{t}^{r}
$$

admits a generic FDR at $y_{0}$.

Proof. We assume that the full stochastic volatility model admits an FDR, and we also assume that (174) is satisfied. We now have to show that, under these assumptions, the parameterized model admits and FDR, i.e. that the Lie algebra (on $\mathcal{H}$ ) of the parameterized model is finite dimensional near $r_{0}$, for every fixed $y$ near $y_{0}$. From Lemma 7.2 we know that the Stratonovich formulation of the parameterized model is given by

$$
d r_{t}=\mu\left(r_{t}, y\right) d t+\sigma\left(r_{t}, y\right) \circ d W_{t}^{r},
$$

which we write as

$$
d r_{t}=\mu^{y}\left(r_{t}\right) d t+\sigma^{y}\left(r_{t}\right) \circ d W_{t}^{r} .
$$

Our task is now to show that

$$
\left\{\mu^{y}, \sigma^{y}\right\}_{L A}
$$

is finite dimensional near $r_{0}$ for all $y$ near $y_{0}$.

Since we assumed that the full model possessed an FDR we know that the Lie algebra

$$
\{\hat{\mu}, \hat{\sigma}, \hat{b}\}_{L A}=\left\{\left[\begin{array}{c}
\mu(r, y) \\
a(y)
\end{array}\right],\left[\begin{array}{c}
\sigma(r, y) \\
0
\end{array}\right],\left[\begin{array}{c}
0 \\
b(y)
\end{array}\right]\right\}_{L A}
$$

is finite dimensional near $\hat{r}_{0}$. We now have the trivial inclusion

$$
\{\hat{b}\}_{L A} \subseteq\{\hat{\mu}, \hat{\sigma}, \hat{b}\}_{L A},
$$


and we go on to compute $\{\hat{b}\}_{L A}=\left\{\hat{b}_{1}, \ldots, \hat{b}_{m_{y}}\right\}_{L A}$. For any $i$ and $j$, let us thus compute the Lie bracket $\left[\hat{b}_{i}, \hat{b}_{j}\right]$. We easily obtain the block matrix form for the Frechet derivative of $\hat{b}_{i}$ on $\hat{\mathcal{H}}$ as

$$
\hat{b}_{i}^{\prime}(y)=\left[\begin{array}{cc}
0 & 0 \\
0 & b_{i}^{\prime}(y)
\end{array}\right]
$$

where $b_{i}^{\prime}$ denotes the Frechet derivative on $R^{k}$ of the vector field $b_{i}$. Performing the same calculation for $\hat{b}_{j}$ we obtain

$$
\left[\hat{b}_{i}, \hat{b}_{j}\right]_{\hat{\mathcal{H}}}=\left[\begin{array}{cc}
0 & 0 \\
0 & b_{i}^{\prime}
\end{array}\right]\left[\begin{array}{c}
0 \\
b_{j}
\end{array}\right]-\left[\begin{array}{cc}
0 & 0 \\
0 & b_{j}^{\prime}
\end{array}\right]\left[\begin{array}{c}
0 \\
b_{i}
\end{array}\right]=\left[\begin{array}{c}
0 \\
{\left[b_{i}, b_{j}\right]_{R^{k}}}
\end{array}\right] .
$$

Continuing in this way by taking repeated brackets, wee see that if $\hat{\beta}$ denotes a generic element of $\{\hat{b}\}_{L A}$ then it has the form

$$
\hat{\beta}=\left[\begin{array}{l}
0 \\
\beta
\end{array}\right]
$$

where $\beta$ denotes a generic element of $\{b\}_{L A}$. We can formally write this as

$$
\{\hat{b}\}_{L A}=\left\{\hat{b}_{1}, \ldots, \hat{b}_{m_{y}}\right\}_{L A}=\left[\begin{array}{c}
0 \\
\left\{b_{1}, \ldots, b_{m_{y}}\right\}_{L A}
\end{array}\right]=\left[\begin{array}{c}
0 \\
\{b\}_{L A}
\end{array}\right]
$$

We assumed that $a \in\{b\}_{L A}$, so there exists vector fields $c_{1}(y), \ldots, c_{n}(y)$ in $\{b\}_{L A}$ and scalar fields $\alpha_{1}(y), \ldots, \alpha_{n}(y)$ on $R^{k}$ such that

$$
a(y)=\sum_{1}^{n} \alpha_{i}(y) c_{i}(y)
$$

for all $y$ near $y_{0}$. Since $\{\hat{b}\}_{L A} \subseteq\{\hat{\mu}, \hat{\sigma}, \hat{b}\}_{L A}$ we see from the above that the vector fields $\hat{c}_{1}, \ldots, \hat{c}_{n}$ where

$$
\hat{c}_{i}=\left[\begin{array}{c}
0 \\
c_{i}(y)
\end{array}\right]
$$

all lie in $\{\hat{\mu}, \hat{\sigma}, \hat{b}\}_{L A}$. From [9] we know that we are allowed to perform Gaussian elimination. More precisely, we may replace $\hat{\mu}$ by $\hat{\mu}-\sum_{1}^{n} \alpha_{i} \hat{c}_{i}$, and we obtain

$$
\hat{\mu}-\sum_{1}^{n} \alpha_{i} \hat{c}_{i}=\left[\begin{array}{l}
\mu \\
a
\end{array}\right]-\sum_{1}^{n} \alpha_{i}\left[\begin{array}{l}
0 \\
c_{i}
\end{array}\right]=\left[\begin{array}{l}
\mu \\
0
\end{array}\right] .
$$

From this we see that the Lie algebra $\{\hat{\mu}, \hat{\sigma}, \hat{b}\}_{L A}$ for the full model is in fact generated by the much simpler system $\hat{m}, \hat{\sigma}$ and $\hat{b}$ where $\hat{m}$ is defined by

$$
\hat{m}=\left[\begin{array}{l}
\mu \\
0
\end{array}\right] .
$$


Since we assumed that $\{\hat{\mu}, \hat{\sigma}, \hat{b}\}_{L A}$ was finite dimensional, then also the smaller Lie algebra

$$
\{\hat{m}, \hat{\sigma}\}_{L A}=\left\{\left[\begin{array}{l}
\mu \\
0
\end{array}\right], \quad\left[\begin{array}{l}
\sigma \\
0
\end{array}\right]\right\}_{L A}
$$

is necessarily also finite dimensional. In computing this latter Lie algebra we may now argue as for $\{b\}_{L A}$ above. Let us, for example, compute the Lie bracket $\left[\hat{m}, \hat{\sigma}_{i}\right]$. We easily obtain

$$
\left[\hat{m}, \hat{\sigma}_{i}\right]=\left[\begin{array}{cc}
\mu_{r} & \mu_{y} \\
0 & 0
\end{array}\right]\left[\begin{array}{c}
\sigma_{i} \\
0
\end{array}\right]-\left[\begin{array}{cc}
\sigma_{i r} & \sigma_{i y} \\
0 & 0
\end{array}\right]\left[\begin{array}{l}
\mu \\
0
\end{array}\right]=\left[\begin{array}{c}
\mu_{r} \sigma_{i}-\sigma_{i r} \mu \\
0
\end{array}\right]
$$

where subindex $r$ and $y$ denotes the partial Frechet derivative w.r.t $r$ and $y$. Now we observe that $\mu_{r}(r, y) \sigma_{i}(r, y)-\sigma_{i r}(r, y) \mu(r, y)=\left[\mu^{y}, \sigma_{i}^{y}\right](r)$ so we have

$$
\left[\hat{m}, \hat{\sigma}_{i}\right](r, y)=\left[\begin{array}{c}
{\left[\mu^{y}, \sigma_{i}^{y}\right]} \\
0
\end{array}\right](r)
$$

and continuing in this way we obtain

$$
\{\hat{m}, \hat{\sigma}\}_{L A}(r, y)=\left\{\left[\begin{array}{l}
\mu \\
0
\end{array}\right], \quad\left[\begin{array}{l}
\sigma \\
0
\end{array}\right]\right\}_{L A}(r, y)=\left[\begin{array}{c}
\left\{\mu^{y}, \sigma^{y}\right\}_{L A} \\
0
\end{array}\right](r)
$$

Since $\{\hat{m}, \hat{\sigma}\}_{L A}$ is finite dimensional for all $(r, y)$ near $\left(r_{0}, y_{0}\right)$ we thus see that $\left\{\mu^{y}, \sigma^{y}\right\}_{L A}$ has to be finite dimensional near $r_{0}$ for all $y$ near $y_{0}$. This however is equivalent to the existence of an FDR for the parameterized model.

We have the following obvious corollary, which seems to be enough for many concrete applications.

Corollary 7.1 Assume that the Lie algebra generated by $b$ in $R^{k}$ is full, i.e. that

$$
\left\{b_{1}, \ldots, b_{m_{y}}\right\}_{L A}=R^{k} \text {. }
$$

Then, regardless of the form of a, the existence of an FDR for the parameterized model is necessary for the existence of an FDR for the full model. In particular, the assumption above is valid, and thus the conclusion holds, for the following special cases.

- $m_{y}=k$ and the $k \times k$ diffusion matrix $b(y)$ is invertible near $y_{0}$.

- y is scalar and driven by a scalar Wiener process (i.e. $k=m_{y}=1$ ), and the scalar field $b(y)$ is nonzero near $y_{0}$.

We now go on to obtain more precise (but still easily verifiable) necessary conditions, and the simplest case is when the diffusion matrix $b$ is square and invertible. Since the multidimensional case is a bit messy we start with the scalar case, and we will in fact use the scalar result in the proof of the multidimensional case. 
Proposition 7.3 Assume that $y$ and $W^{y}$ are scalar and that the (scalar) diffusion term $b(y)$ is nonzero near $y_{0}$. Then the following conditions are necessary for the existence of an FDR for the full model.

- For every fixed $r$ and $y$ near $\left(r_{0}, y_{0}\right)$ the partial derivatives of $\mu$ and $\sigma_{i}(r, y)$ $i=1, \ldots, m_{r}$ w.r.t $y$ span a finite dimensional space in $\mathcal{H}$, i.e. there exists a finite number $N$ such that for every $(r, y)$

$$
\operatorname{dim} \operatorname{span}\left\{\frac{\partial^{n} \mu}{\partial y^{n}}(r, y) ; \quad n=0,1,2, \ldots\right\} \leq N
$$

and

$$
\operatorname{dim} \operatorname{span}\left\{\frac{\partial^{n} \sigma_{i}}{\partial y^{n}}(r, y) ; \quad n=0,1,2, \ldots\right\} \leq N
$$

for every $i=1, \ldots m_{r}$.

- The drift term $\mu$, and each volatility component $\sigma_{i}$ have the form

$$
\mu(r, y, x)=\sum_{j=1}^{n_{0}} c_{0 j}(r, y) \lambda_{0 j}(r, x) .
$$

and

$$
\sigma_{i}(r, y, x)=\sum_{j=1}^{n_{i}} c_{i j}(r, y) \lambda_{i j}(r, x)
$$

Proof. In order to obtain necessary conditions we assume that the full model admits an FDR, and for simplicity of notation we assume that $m_{r}=1$ (this will not affect the proof). The Lie algebra for the full model is then finite dimensional and it is generated by

$$
\hat{\mu}=\left[\begin{array}{c}
\mu(r, y) \\
a(y)
\end{array}\right], \quad \hat{\sigma}=\left[\begin{array}{l}
\sigma \\
0
\end{array}\right], \quad \hat{b}=\left[\begin{array}{c}
0 \\
b(y)
\end{array}\right] .
$$

Since $b$ is scalar and nonzero we can use Gaussian elimination and locally replace $\hat{b}$ by

$$
\frac{1}{b(y)} \hat{b}(y)=\left[\begin{array}{l}
0 \\
1
\end{array}\right],
$$

and, with further elimination, we see that the full Lie algebra is in fact generated by

$$
\hat{\mu}=\left[\begin{array}{c}
\mu(r, y) \\
0
\end{array}\right], \quad \hat{\sigma}=\left[\begin{array}{l}
\sigma \\
0
\end{array}\right], \quad \hat{1}=\left[\begin{array}{l}
0 \\
1
\end{array}\right] .
$$

We start by proving (180), the proof for (179) being identical. Since the full algebra is finite dimensional, also the smaller Lie algebra generated by $\hat{\sigma}$ and $\hat{1}$ 
has to be finite dimensional. In particular the space spanned in $\hat{\mathcal{H}}$ by the vector fields

$$
\hat{\sigma}, \quad[\hat{\sigma}, \hat{1}], \quad[[\hat{\sigma}, \hat{1}], \hat{1}], \quad[[[\hat{\sigma}, \hat{1}], \hat{1}], \hat{1}], \ldots
$$

obtained by starting with $\hat{\sigma}$ and then taking repeated brackets with $\hat{1}$, has to be finite dimensional at every point $(r, y)$ near $\hat{r}_{0}$. We can write these vectors more compactly as

$$
a d_{\hat{1}}^{0}(\sigma), \quad a d_{\hat{1}}^{1}(\sigma), \quad a d_{\hat{1}}^{2}(\sigma), \ldots
$$

where for any vector field $\hat{f}$ the operators $a d_{\hat{f}}^{n}: \hat{\mathcal{H}} \rightarrow \hat{\mathcal{H}}$ are defined recursively by

$$
\begin{aligned}
a d_{\hat{f}}^{0}(\hat{g}) & =\hat{g} \\
a d_{\hat{f}}^{1}(\hat{g}) & =[\hat{g}, \hat{f}], \\
a d_{\hat{f}}^{n+1}(\hat{g}) & =\left[a d_{\hat{f}}^{n}(\hat{g}), \hat{f}\right] .
\end{aligned}
$$

We easily obtain the Frechet derivatives of $\hat{\sigma}$ and $\hat{1}$ as

$$
\hat{\sigma}^{\prime}=\left[\begin{array}{cc}
\partial_{r} \sigma & \partial_{y} \sigma \\
0 & 0
\end{array}\right], \quad \hat{1}^{\prime}=\left[\begin{array}{ll}
0 & 0 \\
0 & 0
\end{array}\right],
$$

where $\partial_{r}$ and $\partial_{r}$ denotes the corresponding partial Frechet derivatives. Thus we have

$$
a d_{\hat{1}}^{1}(\hat{\sigma})=[\hat{\sigma}, \hat{1}]=\left[\begin{array}{cc}
\partial_{r} \sigma & \partial_{y} \sigma \\
0 & 0
\end{array}\right]\left[\begin{array}{l}
0 \\
1
\end{array}\right]-\left[\begin{array}{ll}
0 & 0 \\
0 & 0
\end{array}\right]\left[\begin{array}{l}
\sigma \\
0
\end{array}\right]=\left[\begin{array}{c}
\partial_{y} \sigma \\
0
\end{array}\right]
$$

Similarly we have

$$
\left\{a d_{\hat{1}}^{1}(\hat{\sigma})\right\}^{\prime}=\left[\begin{array}{cc}
\partial_{r} \partial_{y} \sigma & \partial_{y}^{2} \sigma \\
0 & 0
\end{array}\right]
$$

and thus

$a d_{\hat{1}}^{2}(\hat{\sigma})=\left[a d_{\hat{1}^{1}}(\hat{\sigma}), \hat{1}\right]=\left[\begin{array}{cc}\partial_{r} \partial_{y} \sigma & \partial_{y}^{2} \sigma \\ 0 & 0\end{array}\right]\left[\begin{array}{l}0 \\ 1\end{array}\right]-\left[\begin{array}{ll}0 & 0 \\ 0 & 0\end{array}\right]\left[\begin{array}{c}\partial_{y} \sigma \\ 0\end{array}\right]=\left[\begin{array}{c}\partial_{y}^{2} \sigma \\ 0\end{array}\right]$

Continuing this way we see by induction that

$$
a d_{\hat{1}}^{n}(\hat{\sigma})=\left[\begin{array}{c}
\partial_{y}^{n} \sigma \\
0
\end{array}\right] .
$$

Since, by the argument above, $\left\{a d_{\hat{1}}^{n}(\hat{\sigma})(r, y) ; \quad n \geq 0\right\}$ span a finite dimensional subspace of $\hat{\mathcal{H}}$ for all $(r, y)$ near $\hat{r}_{0}$, we thus see that

$$
\left\{\partial_{y}^{n} \sigma(r, y), \quad n \geq 0\right\}
$$


must span a finite dimensional subspace in $\mathcal{H}$ for all $(r, y)$ near $\hat{r}_{0}$. We have thus proved (180) for the case when $W^{r}$ is scalar. The general case is proved by applying the above argument for each component of $\sigma$.

We now go on to prove the necessary condition (182) and we will in fact show that (182) follows from (180). Again we carry out a separate argument for each component $\sigma_{i}$, so without loss of generality we may assume that $\sigma$ only has a single component (i.e that $m_{r}=1$ ). Now, if (180) holds and we denote the dimension of the spanned subspace by $n+1$, there exists scalar fields $a_{j}(r, y) ; \quad j=0, \ldots n$, such that we have the following $\mathcal{H}$-valued vector identity holding locally at $\hat{r}_{0}$

$$
\partial_{y}^{n+1} \sigma(r, y)=\sum_{j=0}^{n} a_{j}(r, y) \partial_{y}^{j} \sigma(r, y)
$$

We now fix an arbitrary $r$, and for this fixed $r$ we define the $\mathcal{H}$-vector functions $Z_{0}(y), Z_{1}(y), \ldots Z_{n}(y)$ by

$$
\begin{aligned}
Z_{0}(y)= & \sigma(r, y), \\
Z_{1}(y)= & \partial_{y} \sigma(r, y), \\
\vdots & \vdots \\
Z_{n}(y)= & \partial_{y}^{n} \sigma(r, y),
\end{aligned}
$$

and the $\mathcal{H}^{n+1}$-valued block vector function $Z(y)$ by

$$
Z(y)=\left[\begin{array}{c}
Z_{0}(y) \\
Z_{1}(y) \\
\vdots \\
Z_{n}(y)
\end{array}\right]
$$

The point of this is that we can now write equation (183) as the linear ODE

$$
\frac{d Z(y)}{d y}=(A(y) \otimes I) Z(y)
$$

where $\otimes$ denotes the Kronecker product, and the $(n+1) \times(n+1)$ matrix function $A$ is defined as the companion matrix

$$
A(y)=\left[\begin{array}{ccccc}
0 & 1 & 0 & \ldots & 0 \\
0 & 0 & 1 & \ldots & 0 \\
\vdots & \vdots & & & 1 \\
a_{0}(y) & a_{1}(y) & a_{2}(y) & \ldots & a_{n}(y)
\end{array}\right] .
$$

As one would perhaps guess, the solution of (184) can be shown to have the representation

$$
Z(y)=\left[\Phi\left(y, y_{0}\right) \otimes I\right] Z\left(y_{0}\right),
$$


where $\Phi$ is the transition matrix induced by $A$. In particular we thus obtain

$$
Z_{0}(y)=\sum_{j=0}^{n} c_{j}(y) Z_{j}\left(y_{0}\right)
$$

where $c_{j}(y)=\Phi\left(y, y_{0}\right)_{1, j}$. Recalling that there is a suppressed $r$ and that $Z_{j}(y)=\partial_{y}^{j} \sigma(r, 0)$ we obtain

$$
\sigma(r, y)=\sum_{j=0}^{n} c_{j}(r, y) \partial_{y}^{j} \sigma(r, 0),
$$

which proves (182). The proof for (181) is identical.

In order to state the corresponding multidimensional result we need to introduce some notation.

Definition 7.4 $A$ multi index $\alpha \in Z_{+}^{k}$ is any $k$-vector with nonnegative integer elements. For a multi index $\alpha=\left(\alpha_{1}, \ldots, \alpha_{k}\right)$ the differential operator $\partial_{y}^{\alpha}$ is defined by

$$
\partial_{y}^{\alpha}=\frac{\partial^{\alpha_{1}}}{\partial y_{1}^{\alpha_{1}}} \frac{\partial^{\alpha_{2}}}{\partial y_{2}^{\alpha_{2}}} \ldots \frac{\partial^{\alpha_{k}}}{\partial y_{k}^{\alpha_{k}}}
$$

We can now state multidimensional version of the theorem above. The crucial assumption needed is that the Lie algebra generated by the diffusion matrix $b(y)$ spans the entire space $R^{k}$. For the proof see [8].

Proposition 7.4 Assume that the condition

$$
\left\{b_{1}, \ldots b_{m_{y}}\right\}_{L A}=R^{k}
$$

is satisfied near $y_{0}$.

Then the following conditions are necessary for the existence of an FDR for the stochastic volatility model.

- For every fixed $r$ and $y$ near $\left(r_{0}, y_{0}\right)$ the partial derivatives of $\mu(r, y)$ and $\sigma_{i}(r, y)$ w.r.t $y$ span a uniformly finite dimensional space in $\mathcal{H}$, i.e. there exists a number $N$ such that for every $(r, y)$

$$
\operatorname{dim} \operatorname{span}\left\{\partial_{y}^{\alpha} \mu(r, y) ; \quad \alpha \in Z_{+}^{k}\right\} \leq N
$$

and

$$
\operatorname{dim} \operatorname{span}\left\{\partial_{y}^{\alpha} \sigma_{i}(r, y) ; \quad \alpha \in Z_{+}^{k}\right\} \leq N
$$

for every $i=1, \ldots m_{r}$. 
- The drift $\mu$ and every volatility component $\sigma_{i}$ have the form

$$
\begin{gathered}
\mu(r, y, x)=\sum_{j=1}^{n_{i}} c_{i j}(r, y) \lambda_{i j}(r, x) . \\
\sigma_{i}(r, y, x)=\sum_{j=1}^{n_{i}} c_{i j}(r, y) \lambda_{i j}(r, x) .
\end{gathered}
$$

\subsubsection{Test examples: II.}

We illustrate the necessary conditions obtained so far by studying the test examples (146)-(149) of Section 7.2. By the assumptions of Section 7.2, all three examples are within the class of orthogonal noise models. We may thus directly apply Proposition 7.2, or (since we have a scalar model) Corollary 7.1 and check whether the corresponding parameterized models possess finite dimensional realizations. In all these cases, however, this test is trivially satisfied since the volatility structures were constructed directly from HJM models possessing short rate realizations. Thus all the models pass this necessary conditions.

We now go on to the necessary conditions of Proposition (7.3). From (182) and ocular inspection of the examples above we immediately have the following result.

Proposition 7.5 Assuming a scalar y-process with non zero diffusion term, the stochastic volatilities in (146), (148) and (149) do not admit an FDR.

Thus (146), (148) and (149) are out of the race. In particular we note that there is no stochastic volatility extension of the CIR forward rate volatility for which there exists a finite dimensional realization. In fact, it is easy to see that we in fact have the following stronger result where we allow both the parameters $a$ and $\sigma$ to depend upon the process $y$.

Proposition 7.6 Consider any stochastic volatility extension of the CIR model of the form

$$
\sigma(r, y, x)=\sigma(y) \sqrt{r(0)} \cdot \lambda(x, \sigma(y), a(y))
$$

where the functions $\sigma(y)$ and $a(y)$ are assumed to be non-constant and where the $y$ process is assumed to have non zero diffusion term. Then the stochastic volatility model does not possess an FDR.

It remains to study the volatility structure (146) in more detail, and this will be done below. 


\subsubsection{Necessary and sufficient conditions}

In this section we provide necessary and sufficient conditions for the existence of an FDR in the case of an orthogonal noise model, thus improving upon the general results of Theorem 7.1.

We need the following definition.

Definition 7.5 Define, for each y, the parameterized Lie algebra $\mathcal{L}^{y}$ on $\mathcal{H}$ by

$$
\mathcal{L}^{y}=\left\{\partial_{y}^{\alpha} \mu^{y}, \partial_{y}^{\alpha} \sigma_{1}^{y}, \ldots, \partial_{y}^{\alpha} \sigma_{m_{r}}^{y} ; \quad \alpha \in Z_{+}^{k}\right\}_{L A}
$$

In this expression $\partial_{y}^{\alpha} \mu^{y}$ is, for each fixed $y$, considered as a (parameterized) vector field on $\mathcal{H}$, and correspondingly for the $\sigma$ components.

In order to obtain reasonably concrete results we need to assume that the Lie algebra generated by the $b$ matrix is full dimensional, leaving the general case as an open problem.

Proposition 7.7 Assume that

$$
\operatorname{dim}\left\{b_{1}, \ldots, b_{m_{y}}\right\}_{L A}=k .
$$

Under this assumption, a necessary and sufficient condition for the existence of an FDR for the stochastic volatility model is that, for each $y$, we have

$$
\operatorname{dim} \mathcal{L}^{y}<\infty
$$

near $r^{0}$.

Proof. From proposition 7.1 we know that there exists an FDR if and only if the Lie algebra $\mathcal{L}$ on $\hat{\mathcal{H}}$ generated by

$$
\left[\begin{array}{c}
\mu(r, y) \\
a(y)
\end{array}\right],\left[\begin{array}{c}
\sigma_{1}(r, y) \\
0
\end{array}\right], \ldots,\left[\begin{array}{c}
\sigma_{m_{r}}(r, y) \\
0
\end{array}\right],\left[\begin{array}{c}
0 \\
b_{1}(y)
\end{array}\right], \ldots,\left[\begin{array}{c}
0 \\
b_{m_{y}}(y)
\end{array}\right]
$$

is finite dimensional. Under the assumption (193), and using Gaussian elimination, we see that $\mathcal{L}$ is generated by

$$
\left[\begin{array}{c}
\mu(r, y) \\
0
\end{array}\right],\left[\begin{array}{c}
\sigma_{1}(r, y) \\
0
\end{array}\right], \ldots,\left[\begin{array}{c}
\sigma_{m_{r}}(r, y) \\
0
\end{array}\right],\left[\begin{array}{c}
0 \\
I_{k}
\end{array}\right]
$$

where $I_{k}$ is the identity matrix on $R^{k}$. Using the fact that repeated bracketing of a vector field of the form

$$
\left[\begin{array}{c}
f(r, y) \\
0
\end{array}\right]
$$


with different columns in

$$
\left[\begin{array}{c}
0 \\
I_{k}
\end{array}\right]
$$

will produce a vector field of the form

$$
\left[\begin{array}{c}
\partial_{y}^{\alpha} f(r, y) \\
0
\end{array}\right]
$$

it now follows that $\mathcal{L}$ is in fact generated by

$$
\left[\begin{array}{c}
\partial_{y}^{\alpha} \mu(r, y) \\
0
\end{array}\right],\left[\begin{array}{c}
\partial_{y}^{\alpha} \sigma(r, y) \\
0
\end{array}\right], \ldots,\left[\begin{array}{c}
\partial_{y}^{\alpha} \sigma_{m_{r}}(r, y) \\
0
\end{array}\right],\left[\begin{array}{c}
0 \\
I_{k}
\end{array}\right] ; \alpha \in Z_{+}^{k}
$$

From this it is clear that $\mathcal{L}$ is generated by

$$
\left[\begin{array}{c}
\mathcal{L}^{y} \\
0
\end{array}\right], \quad\left[\begin{array}{c}
0 \\
I_{k}
\end{array}\right] ; \quad y \in R^{k},
$$

and the proof is finished if we can show that for each multi index $\alpha$ we have

$$
\partial_{y}^{\alpha} \mathcal{L}^{y} \subseteq \mathcal{L}^{y}
$$

It follows by induction that in order to prove (195) we may WLOG assume that $k=1$ (i.e. $y$ is scalar) and that it is in fact enough to prove that

$$
\partial_{y} \mathcal{L}^{y} \subseteq \mathcal{L}^{y}
$$

Now, it is easily seen that

$$
\mathcal{L}^{y}=\bigcup_{k=0}^{\infty} L_{k}^{y}
$$

where

$$
\begin{aligned}
L_{0}^{y} & =\operatorname{span}\left\{\partial_{y}^{n} \mu^{y}, \partial_{y}^{n} \sigma_{1}^{y}, \ldots, \partial_{y}^{n} \sigma_{m_{r}}^{y} ; \quad n \geq 0\right\} \\
L_{k+1}^{y} & =\operatorname{span}\left\{L_{k}^{y},\left[L_{k}^{y}, L_{k}^{y}\right]\right\}, \quad k=0,1, \ldots
\end{aligned}
$$

so it is enough to prove that each $L_{k}^{y}$ is invariant under $\partial_{y}$ and we prove this by induction. The case $k=0$ is clear, so assume that

$$
\partial_{y} L_{n}^{y} \subseteq L_{n}^{y}
$$

for all $n \leq k$. Now fix an arbitrary $f \in L_{k+1}^{y}$. We start by considering two cases: the case when $f \in L_{k}^{y}$ and the case when $f=[g, h]$ with $g, h \in L_{k}^{y}$. If $f \in L_{k}^{y}$ then $\partial_{y} \in L_{k}^{y}$ by the induction assumption, so $\partial_{y} \in L_{k+1}^{y}$. If $f=[g, h]$ with $g, h \in L_{k}^{y}$ then an easy calculation shows that

$$
\partial_{y} f=\left[\partial_{y} g, h\right]+\left[g, \partial_{y} h\right]
$$

which is in $\left[L_{k}^{y}, L_{k}^{y}\right]$ by the induction assumption. Thus also in this case we have $\partial_{y} f \in L_{k+1}^{y}$. A generic $f \in L_{k+1}^{y}$ is, by definition, a linear combination of terms of the above type so we are finished. 


\subsubsection{A simple sufficient condition}

The object of this section is to show that, under some rather restrictive but nontrivial assumptions, it is possible to derive an extremely simple sufficient condition for the existence of an FDR for the full stochastic volatility model in terms of the FDR for the parameterized model. Furthermore; under these assumptions the realization for the full model can be constructed directly, and in a trivial manner, from the realization for the parameterized model.

\section{Assumption 7.4}

1. The Ito formulation of the $r$-dynamics of the stochastic volatility model is of the form

$$
d r_{t}=\mu_{0}\left(r_{t}, y_{t}\right) d t+\sigma_{t}\left(r_{t}, y_{t}\right) d W_{t} .
$$

2. We assume that $y$ is independent of $W$. Apart from this assumption, the process $y$ is allowed to be an arbitrary semimartingale with values in $R^{k}$.

3. For any fixed $y$, the parameterized $r$-model is assumed to possess an FDR of the form

$$
\begin{aligned}
r_{t}^{y} & =G\left(Z_{t}^{y}\right), \\
d Z_{t}^{y} & =A\left(Z_{t}^{y}, y\right) d t+B\left(Z_{t}^{y}, y\right) \circ d W_{t},
\end{aligned}
$$

where $Z^{y}$ is $R^{d}$ valued and $G$ is a smooth mapping $G: R^{d} \rightarrow \mathcal{H}$.

The important part of this assumption is that, for the parameterized model, the parameter $y$ only appears in the $Z^{y}$ dynamics, but not the output mapping $G$. We will discuss the geometric significance of this below, but first we state the result.

Proposition 7.8 Under Assumption 7.4, the stochastic volatility model possesses an FDR, and a concrete realization is in fact given by

$$
\begin{aligned}
r_{t} & =G\left(Z_{t}\right), \\
d Z_{t} & =A\left(Z_{t}, y_{t}\right) d t+B\left(Z_{t}, y_{t}\right) \circ d W_{t},
\end{aligned}
$$

With $G, A$ and $B$ as in (198)-(199).

Proof. From the independence between $y$ and $W$ it follows that the Stratonovich formulation of the $r$-dynamics is given by

$$
d r_{t}=\mu\left(r_{t}, y_{t}\right) d t+\sigma\left(r_{t}, y_{t}\right) \circ d W_{t}
$$

where

$$
\mu(r, y)=\mu_{0}(r, y)-\frac{1}{2} \sigma_{r}(r, y) \sigma(r, y) .
$$


Now let us consider (200)-(201) as an Ansatz. The $r$-dynamics induced by (200)(201) are given by

$$
d r_{t}=G^{\prime}\left(Z_{t}\right) A\left(Z_{t}, y_{t}\right) d t+G^{\prime}\left(Z_{t}\right) B\left(Z_{t}, y_{t}\right) \circ d W_{t},
$$

so it follows that (200)-(201) is a realization of (202) if and only if

$$
\begin{aligned}
& \mu(r, y)=G_{\star} A(r, y), \\
& \sigma(r, y)=G_{\star} B(r, y) .
\end{aligned}
$$

We thus have to prove that (204)-(205) hold, and to this end we use the fact that, by assumption, (198)-(199) is a realization for the parameterized model. The Stratonovich formulation for the parameterized model is easily seen to be given by

$$
d r_{t}^{y}=\mu\left(r_{t}^{y}, y\right) d t+\sigma\left(r_{t}^{y}, y\right) \circ d W_{t},
$$

and the important point here is that this is precisely the parameterized version of the Stratonovich formulation of the original $r$-dynamics. The $r^{y}$-dynamics induced by (198)-(199) are given by

$$
d r_{t}^{y}=G^{\prime}\left(Z_{t}^{y}\right) A\left(Z_{t}^{y}, y\right) d t+G^{\prime}\left(Z_{t}^{y}\right) B\left(Z_{t}^{y}, y\right) \circ d W_{t},
$$

and since this was assumed to be a realization of (206) we thus have

$$
\begin{aligned}
& \mu(r, y)=G_{\star} A(r, y), \\
& \sigma(r, y)=G_{\star} B(r, y),
\end{aligned}
$$

which was to be proved.

Remark 7.1 If the Stratonovich differential in (199) is replaced by an Itô differential i.e. by

$$
d Z_{t}^{y}=A\left(Z_{t}^{y}, y\right) d t+B\left(Z_{t}^{y}, y\right) d W_{t},
$$

then the conclusion of Proposition 7.8 still holds if the Stratonovich differential in (201) is replaced by an Itô differential, i,.e. by

$$
d Z_{t}=A\left(Z_{t}, y_{t}\right) d t+B\left(Z_{t}, y_{t}\right) d W_{t} .
$$

This is useful if the realization of the parameterized model is originally given in Itô form.

This, very strong but also very restrictive, result has a clear and simple geometric interpretation. First, we know from general (orthogonal noise) theory that a necessary condition for an FDR is that the parameterized model possesses an FDR. In general, the realization for the parameterized model will of course be of the form

$$
\begin{aligned}
r_{t}^{y} & =G\left(Z_{t}^{y}, y\right) \\
d Z_{t}^{y} & =A\left(Z_{t}^{y}, y\right) d t+B\left(Z_{t}^{y}, y\right) \circ d W_{t}
\end{aligned}
$$


where the output function $G$ as well as the drift term $A$ and diffusion term $B$ depend upon $y$, but in Proposition 7.8 we have assumed that $G$ does not in fact depend on $y$. To understand the geometric meaning of this assumption we recall from Proposition 4.1 that the parameterized model, for a fixed $y$, admits an FDR if and only if there exists an invariant manifold $\mathcal{G}^{y}$ passing through $r^{0}$, and in the generic case this invariant manifold will of course depend upon $y$. The relation between $\mathcal{G}^{y}$ and the realization (208)-(209) is that

$$
\mathcal{G}^{y}=\operatorname{Im} G^{y}
$$

where the mapping $G^{y}: R^{d} \rightarrow \mathcal{H}$ is defined by $G^{y}(z)=G(z, y)$. Thus; assuming that $G$ does not depend upon the parameter $y$ is equivalent to assuming that the invariant manifold for the parameterized model passing through $r^{0}$ does not depend upon $y$. In that case, denoting the invariant manifold by $\mathcal{G}$ it is of course geometrically obvious that $\mathcal{G} \times R^{k}$ will be a finite dimensional invariant manifold for the process $\left(r_{t}, y_{t}\right)$ thus guaranteeing the existence of an FDR for the full model.

Furthermore, it follows from Proposition 4.2 that the invariant manifold $\mathcal{G}^{y}$ is determined uniquely by the parameterized Lie algebra

$$
\mathcal{L}^{y}=\left\{\mu^{y}, \sigma_{1}^{y}, \ldots, \sigma_{m_{r}}^{y}\right\}_{L A},
$$

so if $\mathcal{L}^{y}$ does not depend upon $y$ then neither will $G(z, y)$. We thus have the following result.

Proposition 7.9 Assume that

- The process $y$ is an $R^{k}$-valued semimartingale which is independent of $W$.

- The parameterized model admits an FDR for every fixed $y$.

- Lie algebra $\mathcal{L}^{y}$ defined in (210) does not depend upon the parameter y.

Then the full model will possess an FDR.

We finish this discussion by noticing that for the general Lie algebraic machinery to work it is essential that all processes are Wiener driven. The geometric reason for this is that the Wiener process acts locally in space (the infinitesimal generator is a partial differential operator) and this allows us to analyze the realization problems using differential geometry (i.e. local analysis). It is therefore noteworthy that in the simple situation discussed above in this section, we did not have to assume that $y$ is driven by a Wiener process - it can also have jumps. 


\subsubsection{An example}

As an application of the results in Section 7.4.5, we consider the following volatility structure for a standard forward rate model driven by a scalar Wiener process $W^{r}$,

$$
\sigma(r, x)=\varphi(r) e^{-\alpha x} .
$$

Here $\varphi$ is assumed to be an arbitrarily chosen smooth scalar field, and $\alpha$ is a positive constant. This is an extension of the model investigated in [35], where an FDR was constructed for the case when $\varphi$ was assumed to be of the particular form $\varphi(r)=g(r(0))$, for some smooth function $g: R \rightarrow R$. As was shown in Section 5.3, the model admits an FDR of the following form.

Define the mapping $G: R_{+} \times R^{2} \rightarrow \mathcal{H}$ by

$$
G\left(t, z_{1}, z_{2}\right)(x)=r^{0}(x+t)+z_{1} e^{-\alpha x}+z_{2} e^{-2 \alpha x}
$$

The realization is then given by

$$
\begin{aligned}
r_{t}(x) & =G\left(t, Z_{1}(t), Z_{2}(t)\right)(x), \\
d Z_{1}(t) & =\left\{\frac{1}{\alpha} \varphi^{2}\left[G_{t}\right]-\alpha Z_{1}(t)\right\} d t+\varphi\left[G_{t}\right] d W_{t}^{r}, \\
d Z_{2}(t) & =-\left\{2 \alpha Z_{1}(t)+\frac{1}{\alpha} \varphi^{2}\left[G_{t}\right]\right\} d t .
\end{aligned}
$$

where we have used the shorthand notation

$$
G_{t}=G\left(t, Z_{1}(t), Z_{2}(t)\right) .
$$

The important point to notice is that the mapping $G$ in (212) does not involve $\varphi$. We may now extend the model above to a stochastic volatility model with an arbitrary scalar $y$-process (assumed to be independent of $W^{r}$ ), by defining the volatility structure as

$$
\sigma(r, y, x)=\varphi(r, y) e^{-\alpha x} .
$$

where $\varphi$ is an arbitrarily chosen scalar field.

By construction, the parameterized model admits an FDR of the form (213)(215) where $G$ is exactly as above, and where $\varphi\left[G_{t}\right]$ is replaced by $\varphi\left[G_{t}, y\right]$. The point is again that $G$ does not involve $y$, so it now follows immediately from Proposition 7.8 that a realization for the stochastic volatility model is given by

$$
\begin{aligned}
r_{t}(x) & =G\left(t, Z_{1}(t), Z_{2}(t)\right)(x), \\
d Z_{1}(t) & =\left\{\frac{1}{\alpha} \varphi^{2}\left[G_{t}, y_{t}\right]-\alpha Z_{1}(t)\right\} d t \\
& +\varphi\left[G_{t}, y_{t}\right] d W_{t}^{r}, \\
d Z_{2}(t) & =-\left\{2 \alpha Z_{1}(t)+\frac{1}{\alpha} \varphi^{2}\left[G_{t}, y_{t}\right]\right\} d t .
\end{aligned}
$$


Remark 7.2 In this example we have used the Itô dynamics instead of the Stratonovich dynamics. The reason is that the Itô dynamics of the realization are simpler than the Stratonovich dynamics.

\subsection{Forward rate stochastic volatility models}

We now go on to apply the general results above to the more concrete case of forward rate models. we recall that the Ito formulation of the stochastic volatility forward rate model is given by

$$
\begin{aligned}
d r_{t}(x) & =\left\{\frac{\partial}{\partial x} r_{t}(x)+\mathbf{H} \sigma\left(r_{t}, y_{t}, x\right)\right\} d t+\sigma\left(r_{t}, y_{t}, x\right) d W_{t} \\
d y_{t} & =a^{0}\left(y_{t}\right) d t+b\left(y_{t}\right) d W_{t},
\end{aligned}
$$

where $\mathbf{H}$ is defined in (137). On Stratonovich form the model has the form

$$
\begin{aligned}
d r_{t} & =\mu\left(r_{t}, y_{t}\right) d t+\sigma\left(r_{t}, y_{t}\right) \circ d W_{t} \\
d y_{t} & =a\left(y_{t}\right) d t+b\left(y_{t}\right) \circ d W_{t},
\end{aligned}
$$

where

$$
\begin{aligned}
\mu(r, y) & =\mathbf{F} r+\mathbf{H} \sigma(r, y)-\frac{1}{2} \sigma_{r}(r, y) \sigma(r, y)-\frac{1}{2} \sigma_{y}(r, y) b(y) \\
a(y) & =a^{0}(y)-\frac{1}{2} b_{y}(y) b(y) .
\end{aligned}
$$

As usual $\mathbf{F}$ denotes the operator $\frac{\partial}{\partial x}, \sigma_{r}$ denotes the partial Frechet derivative of $\sigma$ w.r.t. the vector variable $r$ and similarly for $\sigma_{y}$.

\subsubsection{Necessary conditions for orthogonal noise models}

In the orthogonal noise case the model has the following Stratonovich form

$$
\begin{aligned}
d r_{t} & =\mu\left(r_{t}, y_{t}\right) d t+\sigma\left(r_{t}, y_{t}\right) \circ d W_{t}^{r} \\
d y_{t} & =a\left(y_{t}\right) d t+b\left(y_{t}\right) \circ d W_{t}^{y},
\end{aligned}
$$

where

$$
\begin{aligned}
\mu(r, y) & =\mathbf{F} r+\mathbf{H} \sigma(r, y)-\frac{1}{2} \sigma_{r}(r, y) \sigma(r, y) \\
a(y) & =a^{0}(y)-\frac{1}{2} b_{y}(y) b(y) .
\end{aligned}
$$

We now have the following surprisingly restrictive result. 
Proposition 7.10 Assume the following:

- The model is an orthogonal noise model.

- The condition

$$
\left\{b_{1}, \ldots b_{m_{y}}\right\}_{L A}=R^{k}
$$

is satisfied near $y^{0}$.

Then, a necessary condition for the existence of an FDR is that the volatility structure has the form

$$
\sigma_{i}(r, y, x)=\sum_{j=1}^{N} \varphi_{i j}(r, y) \lambda_{j}(x), \quad i=1, \ldots, m_{r},
$$

where $\lambda_{1}, \ldots, \lambda_{N}$ are constant vector fields, and $\varphi_{i j}$ are smooth scalar fields.

Proof. Since we have assumed orthogonal noise, Proposition 7.2 implies that a necessary condition for the existence of an FDR is that the parameterized model admits an FDR. Furthermore; applying Theorem 4.13 of [23] to the parameterized model it follows that the volatility must be of the form

$$
\sigma_{i}(r, y, x)=\sum_{j=1}^{N} \varphi_{i j}(r, y) \lambda_{j}(y, x) .
$$

Given this expression, an application of Proposition 7.4 finishes the proof.

Given a volatility structure of the form (228) we now go on to find sufficient conditions for the existence of an FDR.

\subsubsection{Sufficient conditions for the general noise models}

We now consider a multidimensional forward rate model of the form

$$
\begin{aligned}
d r_{t} & =\mu\left(r_{t}, y_{t}\right) d t+\sigma\left(r_{t}, y_{t}\right) \circ d W_{t} \\
d y_{t} & =a\left(y_{t}\right) d t+b\left(y_{t}\right) \circ d W_{t} .
\end{aligned}
$$

where $W$ is assumed to be $m$-dimensional, and $y$ is as usual $k$-dimensional. We will assume that the volatility structure is of the form (228), but we stress the fact that we do not restrict ourselves to the orthogonal noise model.

We recall from Section 2.2 that a real valued function $f: R \rightarrow R$ is said to be quasi exponential if it is the solution of a linear ODE with constant coefficients, alternatively that it can be written as

$$
f(x)=\sum_{i} e^{\gamma_{i} x}+\sum_{j} e^{\alpha_{j} x}\left[p_{j}(x) \cos \left(\omega_{j} x\right)+q_{j}(x) \sin \left(\omega_{j} x\right)\right],
$$


where $\gamma_{i}, \alpha_{j}, \omega_{j}$ are real numbers, whereas $p_{j}$ and $q_{j}$ are real polynomials.

The main result is as follows.

Proposition 7.11 Consider the model (230)-(231) and assume that the components of $\sigma$ are of the form

$$
\sigma_{i}(r, y, x)=\sum_{j=1}^{N} \varphi_{i j}(r, y) \lambda_{j}(x), \quad i=1, \ldots, m .
$$

Under this assumption a sufficient condition for the existence of an FDR is that $\lambda_{1}(x), \ldots, \lambda_{m}(x)$ are quasi exponential. The scalar fields $\varphi_{i j}(x)$ are allowed to be arbitrary.

Proof. In order to avoid to much and messy notation, we give the proof only for the simplified case when

$$
\sigma_{i}(r, y, x)=\varphi_{i}(r, y) \lambda_{i}(x)
$$

The arguments in the general case are almost identical. Under the given assumption the Stratonovich drift term of $r$ is given by

$$
\mu=\mathbf{F} r+\sum_{i=1}^{m} \Phi_{i} D_{i}-\frac{1}{2} \sum_{i=1}^{m} \varphi_{i r}\left[\lambda_{i}\right] \varphi_{i} \lambda_{i}-\frac{1}{2} \sum_{i=1}^{m} \varphi_{i y}\left[b_{i}\right] \lambda_{i}
$$

where $b_{i}$ denotes the i.th column of the matrix $b$. The Lie algebra $\mathcal{L}$ under study is the one generated by the vector fields

$$
\left[\begin{array}{c}
\mu \\
a
\end{array}\right],\left[\begin{array}{c}
\varphi_{1} \lambda_{1} \\
b_{1}
\end{array}\right], \ldots,\left[\begin{array}{c}
\varphi_{m} \lambda_{m} \\
b_{m}
\end{array}\right]
$$

Obviously, $\mathcal{L}$ is included in the larger algebra $\mathcal{L}_{1}$, generated by

$$
\left[\begin{array}{c}
\mu \\
0
\end{array}\right],\left[\begin{array}{c}
\varphi_{1} \lambda_{1} \\
0
\end{array}\right], \ldots,\left[\begin{array}{c}
\varphi_{m} \lambda_{m} \\
0
\end{array}\right],\left[\begin{array}{l}
0 \\
a
\end{array}\right],\left[\begin{array}{c}
0 \\
b_{1}
\end{array}\right], \ldots,\left[\begin{array}{c}
0 \\
b_{m}
\end{array}\right] .
$$

Using the structure of $\mu$ we can reduce this generator system to

$$
\left[\begin{array}{c}
\mathbf{F} r+\sum_{i=1}^{m} \Phi_{i} D_{i} \\
0
\end{array}\right],\left[\begin{array}{c}
\lambda_{1} \\
0
\end{array}\right], \ldots,\left[\begin{array}{c}
\lambda_{m} \\
0
\end{array}\right],\left[\begin{array}{l}
0 \\
a
\end{array}\right],\left[\begin{array}{l}
0 \\
b
\end{array}\right] .
$$

From this we see that $\mathcal{L}_{1}$ is included in the algebra $\mathcal{L}_{2}$, generated by

$$
\left[\begin{array}{c}
\mathbf{F} r \\
0
\end{array}\right],\left[\begin{array}{c}
D_{1} \\
0
\end{array}\right], \ldots,\left[\begin{array}{c}
D_{m} \\
0
\end{array}\right],\left[\begin{array}{c}
\lambda_{1} \\
0
\end{array}\right], \ldots,\left[\begin{array}{c}
\lambda_{m} \\
0
\end{array}\right],\left[\begin{array}{l}
0 \\
a
\end{array}\right],\left[\begin{array}{l}
0 \\
b
\end{array}\right]
$$

As in Section 4.5.1 it now follows that $\mathcal{L}_{2}$ is finite dimensional if and only if $\lambda_{1}, \ldots, \lambda_{m}$ are quasi exponential. 


\subsubsection{The scalar case}

We finish by a reasonably complete investigation of the most important special case, which occurs when $y$ is scalar, $r$ and $y$ are driven by scalar Wiener processes, and the volatility has the form

$$
\sigma(r, y, x)=\varphi(r, y) \lambda(x) .
$$

Such a model will have the form

$$
\begin{aligned}
d r_{t}(x) & =\left\{\mathbf{F} r_{t}(x)+\Phi(r, y) D(x)\right\} d t+\varphi(r, y) \lambda(x) d W_{t}^{r} \\
d y_{t} & =a^{0}\left(y_{t}\right) d t+b\left(y_{t}\right) d W_{t}^{y} .
\end{aligned}
$$

where

$$
\begin{aligned}
\Phi(r, y) & =\varphi^{2}(r, y), \\
D(x) & =\lambda(x) \int_{0}^{x} \lambda(s) d s .
\end{aligned}
$$

In order to allow for a correlation, $\rho$, between $W^{r}$ and $W^{y}$ we write them as

$$
\begin{aligned}
W_{t}^{r} & =\rho W_{t}^{1}+\sqrt{1-\rho^{2}} W_{t}^{2}, \\
W_{t}^{y} & =W_{t}^{1}
\end{aligned}
$$

where $W^{1}$ and $W^{2}$ are independent Wiener processes. We then have the dynamics

$$
\begin{aligned}
& d r_{t}=\left\{\mathbf{F} r_{t}+\Phi D\right\} d t+\varphi \lambda \rho W_{t}^{1}+\varphi \lambda \sqrt{1-\rho^{2}} W_{t}^{2} \\
& d y_{t}=a^{0} d t+b d W_{t}^{1} .
\end{aligned}
$$

We can now prove the following main result for the scalar case.

Proposition 7.12 Assume that $\varphi_{y}(r, y) \neq 0$, and that $b(y) \neq 0$ i.e. that the model is non trivial. Then the following hold.

- In the non-perfectly correlated case $|\rho|<1$, a necessary and sufficient condition for the existence of an FDR is that the vector field $\lambda$ is quasi exponential. The scalar field $\varphi(r, y)$ is allowed to be arbitrary.

- In the perfectly correlated case $|\rho|=1$, the condition above is sufficient.

Proof. The Stratonovich dynamics of the model are given by

$$
\begin{aligned}
& d r_{t}=\left\{\mathbf{F} r_{t}+\Phi D-\frac{1}{2} \varphi_{r}[\lambda] \varphi \lambda-\frac{1}{2} \varphi_{y} b \lambda\right\} d t+\varphi \lambda \circ W_{t}^{1}+\sqrt{1-\rho^{2}} \varphi \lambda \circ W_{t}^{2} \\
& d y_{t}=a d t+b \circ d W_{t}^{1} .
\end{aligned}
$$


Thus the relevant Lie algebra $\mathcal{L}$ on $\hat{\mathcal{H}}$ is generated by the vector fields

$$
\left[\begin{array}{c}
\mathbf{F} r+\Phi D-\frac{1}{2} \varphi_{r}[\lambda] \varphi \lambda-\frac{1}{2} \varphi_{y} b \lambda \\
a
\end{array}\right],\left[\begin{array}{c}
\rho \varphi \lambda \\
b
\end{array}\right],\left[\begin{array}{c}
\sqrt{1-\rho^{2}} \varphi \lambda \\
0
\end{array}\right],
$$

We start with the non-perfectly correlated case, so we assume that $|\rho|<1$. Then, by Gaussian elimination, the system of generators can immediately be reduced to

$$
\left[\begin{array}{c}
\mathbf{F} r+\Phi D \\
0
\end{array}\right],\left[\begin{array}{l}
0 \\
1
\end{array}\right],\left[\begin{array}{l}
\lambda \\
0
\end{array}\right]
$$

The Lie bracket between the first two vector fields gives us

$$
\left[\begin{array}{c}
\Phi_{y} D \\
0
\end{array}\right]
$$

so after reducing this field we have the generators

$$
\left[\begin{array}{c}
\mathbf{F} r+\Phi D \\
0
\end{array}\right],\left[\begin{array}{l}
D \\
0
\end{array}\right],\left[\begin{array}{l}
\lambda \\
0
\end{array}\right],\left[\begin{array}{l}
0 \\
1
\end{array}\right]
$$

which finally reduce to

$$
\left[\begin{array}{c}
\mathbf{F} r \\
0
\end{array}\right],\left[\begin{array}{l}
D \\
0
\end{array}\right],\left[\begin{array}{l}
\lambda \\
0
\end{array}\right],\left[\begin{array}{l}
0 \\
1
\end{array}\right]
$$

From this it follows immediately that the Lie algebra is finite dimensional if an only if the linear span of

$$
\left\{\mathbf{F}^{n} \lambda, \quad \mathbf{F}^{n} D ; \quad n \geq 0\right\}
$$

is a finite dimensional subspace in $\mathcal{H}$. It is however easily seen that this happens if and only if $\lambda$ is quasi exponential.

In the perfectly correlated case $|\rho|=1$ we can WLOG assume that $\rho=1$ and we are left with the following generators for the Lie algebra $\mathcal{L}$.

$$
\left[\begin{array}{c}
\mathbf{F} r+\Phi D-\frac{1}{2} \varphi_{r}[\lambda] \varphi \lambda-\frac{1}{2} \varphi_{y} b \lambda \\
a
\end{array}\right],\left[\begin{array}{c}
\varphi \lambda \\
b
\end{array}\right]
$$

There seems tho be no easy way of reducing this set of generators, but it is obvious that $\mathcal{L}$ is included in the Lie algebra $\mathcal{L}_{\text {ext }}$ generated by the fields

$$
\left[\begin{array}{c}
\mathbf{F} r+\Phi D-\frac{1}{2} \varphi_{r}[\lambda] \varphi \lambda-\frac{1}{2} \varphi_{y} b \lambda \\
a
\end{array}\right],\left[\begin{array}{c}
\varphi \lambda \\
0
\end{array}\right],\left[\begin{array}{l}
0 \\
b
\end{array}\right]
$$

Thus a sufficient condition for an FDR is that the larger Lie algebra $\mathcal{L}_{\text {ext }}$ is finite dimensional. It is however easily seen that $\mathcal{L}_{\text {ext }}$ is identical with the algebra discussed in the non-perfectly correlated case above, so we are finished. 


\subsubsection{Test examples: III.}

We can now continue our study of the test examples of Section 7.2. In fact, only one example is left in the race, namely

\section{HW with stochastic $\sigma$ :}

$$
\sigma(r, y, x)=y e^{-a x} .
$$

We now have the following result, which is immediately obtained from Proposition 7.12 .

Proposition 7.13 The stochastic volatility version of the Hull-White extended Vasiček volatility structure with stochastic $\sigma$, as in (236) admits an FDR.

\subsubsection{Construction of realizations}

In the previous sections we have provided existence results for FDRs, but so far we have not actually constructed any concrete realizations. However; the construction technique outlined in Section 5 can immediately be adapted to the stochastic volatility framework, and we only give an illustrative example. The example is the Hull-White extended Vasiček model with stochastic $\sigma$ as in (236) above. We already know that the forward rate model of the form (230)-(231), with volatilities given by (236), has a finite dimensional realization. Not surprisingly, $y$ can be chosen as one of the state variables, and a concrete realization can be shown to be given by

$$
\hat{r}_{t}=\widehat{G}\left(Z_{t}, y_{t}\right) .
$$

Here $\widehat{G}$ is defined by

$$
\widehat{G}\left(z_{0}, z_{1}, z_{2}, y\right)=\left[\begin{array}{c}
G\left(z_{0}, z_{1}, z_{2}, y\right) \\
y_{0}+y
\end{array}\right],
$$

where $G$ is given by

$$
G\left(z_{0}, z_{1}, z_{2}, y\right)(x)=r_{0}\left(x+z_{0}\right)+e^{-\alpha x} z_{1}-\frac{e^{-2 \alpha x}}{\alpha} z_{2} .
$$

The dynamics of the state space variables are given by

$$
\left\{\begin{aligned}
d Z_{0} & =d t \\
d Z_{1} & \left.=\left[-\alpha Z_{1}+\frac{1}{\alpha}\left(y_{0}+y\right)^{2}\right)\right] d t+\left(y_{0}+y\right) d W_{t}, \\
d Z_{2} & =\left[-2 \alpha Z_{2}+\left(y_{0}+y\right)^{2}\right] d t \\
d y & =a_{0}(y) d t+b(y) d W_{t} .
\end{aligned}\right.
$$

Here $a_{0}(y)=a(y)+\frac{1}{2} b_{y}(y) b(y)$. 


\section{References}

[1] Bhar, R., And Chiarella, C. Transformation of Heath-Jarrow-Morton models to Markovian systems. The European Journal of Finance 3 (1997), $1-26$.

[2] Buörk, T. Arbitrage Theory in Continuous Time. Oxford University Press, 1998.

[3] Björk, T. A geometric view of interest rate theory. In Option Pricing, Interest Rates and Risk Management., E. Jouini, J. Cvitanic, and M. Musiela, Eds. Cambridge University Press, 2001.

[4] BjöRK, T. Interest rate theory. In Financial Mathematics, Springer Lecture Notes in Mathematics, Vol 1656, W. Runggaldier, Ed. Springer Verlag, 2001.

[5] BjÖRK, T., AND CRISTEnsen, B. Interest rate dynamics and consistent forward rate curves. Mathematical Finance 9, 4 (1999), 323-348.

[6] Björk, T., And Gombani, A. Minimal realizations of interest rate models. Finance and Stochastics 3, 4 (1999), 413-432.

[7] BJÖRK, T., AND LANDÉN, C. On the construction of finite dimensional realizations for nonlinear forward rate models. Finance and Stochastics 6 , 3 (2002), 303-331.

[8] Buörk, T., Landén, C., And Svensson, L. Finite dimensional markovian realizations for stochastic volatility forward rate models. Forthcoming in: Proceedings of the Royal Society, 2002.

[9] Björk, T., And Svensson, L. On the existence of finite dimensional realizations for nonlinear forward rate models. Mathematical Finance 11, 2 (2001), 205-243.

[10] Brace, A., And Musiela, M. A multifactor Gauss Markov implementation of Heath, Jarrow, and Morton. Mathematical Finance 4 (1994), 259-283.

[11] Brocket, P. Finite Dimensional Linear Systems. Wiley, 1970.

[12] Brocket, P. Nonlinear systems and nonlinear estimation theory. In Stochastic systems: The mathematics of filtering and identification and applications, M. Hazewinkel and J. Willems, Eds. Reidel, 1981.

[13] Carverhill, A. When is the spot rate Markovian? Mathematical Finance 4 (1994), 305-312.

[14] Chinkella, C., And Kwon, O. K. Forward rate dependent Markovian transformations of the Heath-Jarrow-Morton term structure model. Finance and Stochastics 5 (2001), 237-257. 
[15] Cox, J., Ingersoll, J., And Ross, S. A theory of the term structure of interest rates. Econometrica 53 (1985), 385-408.

[16] Da Prato, G., And Zabzcyk, J. Stochastic Equations in Infinite Dimensions. Cambridge University Press, 1992.

[17] Duffie, D., And Kan, R. A yield factor model of interest rates. Mathematical Finance 6, 4 (1996), 379-406.

[18] Eberlein, E., And Raible, S. Term structure models driven by general Levy processes. Mathematical Finance 9, 1 (1999), 31-53.

[19] Filipović, D. A note on the nelson-siegel family. Mathematical Finance 9, 4 (1999), 349-359.

[20] Filipović, D. Exponential-polynomial families and the term structure of interest rates. Bernoulli 6 (2000), 1-27.

[21] Filipović, D. Invariant manifolds for weak solutions of stochastic equations. Probability Theory and Related Fields 118 (2000), 323-341.

[22] Filipović, D. Consistency Problems for Heath-Jarrow-Morton Interest Rate Models. Springer Lecture Notes in Mathematics, Vol. 1760. Springer Verlag., 2001.

[23] Filipović, D., and Teichmann, J. Existence of finite dimensional realizations for stochastic equations, 2001. Forthcoming in J. Funct. Anal.

[24] Filipović, D., and Teichmann, J. On finite dimensional term structure models, 2002. Working paper.

[25] Filipović, D., And Teichmann, J. On the geometry of the term structure of interest rates, 2003. To appear in Proceedings of the Royal Society.

[26] Heath, D., Jarrow, R., and Morton, A. Bond pricing and the term structure of interest rates: a new methodology for contingent claims valuation. Econometrica 60 (1992), 77-105.

[27] Ho, T., AND LeE, S. Term structure movements and pricing interest rate contingent claims. Journal of Finance 41 (1986), 1011-1029.

[28] Hull, J., And White, A. Pricing interest-rate-derivative securities. Review of Financial Studies 3 (1990), 573-592.

[29] Inui, K., ANd KiJima, M. A Markovian framework in multi-factor HeathJarrow-Morton models. Journal of Financial and Quantitative Analysis 33 (1998), 423-440.

[30] IsIDoRI, A. Nonlinear Control Systems. Springer Verlag, 1989. 
[31] Jeffrey, A. Single factor Heath-Jarrow-Morton term structure models based on Markov spot interest rate dynamics. Journal of Financial and Quantitative Analysis 30 (1995), 619-642.

[32] Musiela, M. Stochastic PDE:s and term structure models. Preprint, 1993.

[33] Musiela, M., And Rutkowski, M. Martingale Methods in Financial Modelling. Springer-Verlag, 1997.

[34] Nelson, C., And Siegel, A. Parsimonious modelling of yield curves. Journal of Business 60 (1987), 473-489.

[35] Ritchien, P., and Sankarasubramanian, L. Volatility structures of forward rates and the dynamics of the term structure. Mathematical Finance 5, 1 (1995), 55-72.

[36] VASIČEK, O. An equilibrium characterization of the term stucture. Journal of Financial Economics 5 (1977), 177-188.

[37] WARner, F. Foundations of differentiable manifolds and Lie groups. Scott, Foresman, Hill, 1979.

[38] ZABCZYK, J. Stochastic invariance and conistency of financial models. Preprint. Scuola Normale Superiore, Pisa, 2001. 